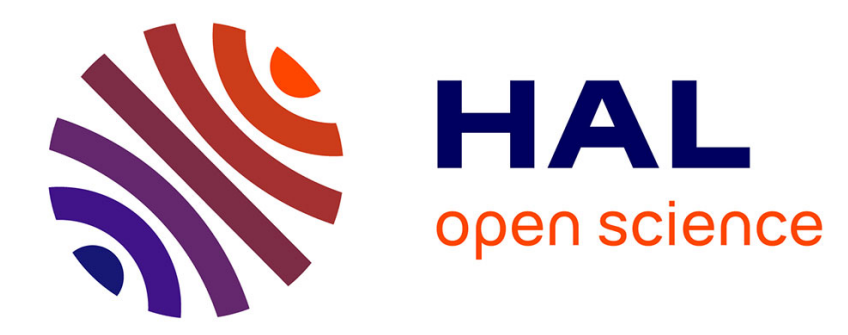

\title{
(A)symétrie et convergence des chocs macroéconomiques en Asie de l'Est: une analyse dynamique
}

\author{
Cyriac Guillaumin
}

\section{To cite this version:}

Cyriac Guillaumin. (A)symétrie et convergence des chocs macroéconomiques en Asie de l'Est: une analyse dynamique. 6ème Journée d'Econométrie, Nov 2007, Nanterre, France. hal-00192626

\section{HAL Id: hal-00192626 \\ https://hal.science/hal-00192626}

Submitted on 29 Nov 2007

HAL is a multi-disciplinary open access archive for the deposit and dissemination of scientific research documents, whether they are published or not. The documents may come from teaching and research institutions in France or abroad, or from public or private research centers.
L'archive ouverte pluridisciplinaire HAL, est destinée au dépôt et à la diffusion de documents scientifiques de niveau recherche, publiés ou non, émanant des établissements d'enseignement et de recherche français ou étrangers, des laboratoires publics ou privés. 


\title{
(A)symétrie et convergence des chocs macroéconomiques en Asie de l'Est : une analyse dynamique
}

\author{
Cyriac Guillaumin ${ }^{1}$
}

Résumé: L'objectif de ce papier est d'identifier le degré d'(a)symétrie des chocs macroéconomiques et d'examiner leur évolution au fil du temps pour les pays de l'Asean+3 afin de mieux comprendre les choix en terme de régime de change. Notre analyse s'effectue en deux temps. Dans un premier temps, nous utilisons un processus VAR structurel (SVAR) pour décomposer, selon la méthodologie de Blanchard et Quah (1989), les chocs macroéconomiques puis déterminer leur degré de corrélation. Toutefois, les coefficients de corrélation ne permettent pas de juger l'évolution de l'(a)symétrie des chocs. Ainsi, le calcul des coefficients de corrélation inconditionnelle entre les chocs subit un défaut d'invariance structurelle adressant une critique de Lucas (1976) à la plupart des travaux en termes de symétrie des chocs au sujet de la dynamique des zones monétaires optimales. Pour tenter de corriger ce problème, nous élargissons l'analyse des chocs d'offre et de demande en mesurant une corrélation dans le temps comme le propose Boone (1997) permettant ainsi d'observer l'évolution de la convergence des structures entre économies de l'Asean +3 . Pour cela, nous utilisons une méthodologie dynamique basée sur le filtre de Kalman. Les résultats montrent qu'il n'y a pas de convergence globale au niveau de la zone. Cependant, une analyse plus spécifique montre qu'il existe une convergence relative des chocs de demande entre certains pays. Nos résultats font également apparaître que la crise financière de 1997 n'a pas eu les mêmes effets sur les pays de l'Asean+3 et qu'il y a un avant et un après crise.

Mots-clefs : (a)symétrie des chocs, convergence des cycles économiques, VAR structurels, filtre de Kalman, Asean+3.

Classification JEL : E32, F15, F33.

\footnotetext{
${ }^{1}$ CEPN, université Paris 13, 99 avenue Jean-Baptiste Clément, 93430 Villetaneuse. Tel/Fax : 01494032 55/ 01494033 34.Courriel : cyriac.guillaumin@edu.univ-paris13.fr

Je tiens à remercier chaleureusement Virginie Coudert et Cuong Le Van pour leurs précieux commentaires et leur soutien. Je suis redevable envers Laurence Boone et Claude Chambon. Je remercie Sopanha Sa et Marc Dubert pour leurs commentaires sur une première version de ce papier.

Je remercie également les participants du séminaire de l'EPEE (université d'Evry-Val d'Essonne) ainsi que ceux des 24èmes journées internationales d'économie monétaire et bancaire organisées par le GDR européen Monnaie, Banque, Finance à l'université Rennes 1.
} 


\section{Introduction}

La théorie des zones monétaires optimales, développée, essentiellement, par Mundell (1961), McKinnon (1963) et Kenen (1969), peut guider dans le choix d'un régime de change en s'intéressant notamment au degré d'asymétrie des chocs. Supposons deux économies. Si les cycles de ces deux économies sont synchronisés, nous pouvons alors faire l'hypothèse qu'ils sont touchés par des chocs symétriques (ou communs). Ces deux pays ont alors moins besoin de faire varier leur taux de change, que si leurs cycles n'étaient pas synchronisés. Ainsi, la convergence des cycles favorise un régime de change davantage fixe (avec coopération) par rapport à un régime de change flexible. Un régime de change flottant peut aussi être adopté en cas de chocs symétriques, avec coordination ; mais, la coordination est un processus coûteux où la « tentation de dévier » existe. Il n'existe pas de régime de change optimal dans l'absolu, susceptible de convenir à tous les pays ou à toutes les époques, ne serait-ce que parce que les circonstances évoluent (Frankel, 1999). Dès lors, « le choix d'un régime de change dépend de différents critères qu'il est nécessaire de pondérer de manière différente selon la région du monde et selon les périodes » (Bénassy-Quéré et alii, 2004).

Depuis l'effondrement des régimes de changes fixes (qu'ils soient de facto ou non) ancrés sur le dollar en 1997, il y a une véritable question sur le choix du régime de change dans certains pays d'Asie de l'Est. Depuis quelques années, l'émergence de la Chine et son rôle très actif, avec le Japon, dans l'accumulation de réserves de change ${ }^{2}$ relancent ce débat. A la veille de la crise financière, la plupart des pays d'Asie de l'Est avaient un régime de change fixe (Reinhart et Rogoff, 2002). Après cette crise, et même si le Fonds Monétaire International a préconisé davantage de flexibilité, certains pays, d'une manière officielle ou non, ont continué d'avoir un taux de change plus ou moins fixe (Dubert et Coudert, 2004 et Levy-Yeyati et Sturzenegger, 2005). Afin de prévenir les crises, les pays de l'Asean ${ }^{3}$ ainsi que la Chine, le Japon et la Corée du Sud ${ }^{4}$ ont conclu un accord : les accords de Chiang Mai. Ces accords prennent la forme de swaps qui permettront à une banque centrale dont la monnaie est en difficulté d'échanger pendant quelques temps sa monnaie contre des réserves des banques centrales participant à l'accord pour intervenir sur son marché des changes. Toutefois, il

\footnotetext{
${ }^{2}$ En mars 2007, les réserves de change chinoises, massivement réinvesties en titres du Trésor américain, atteignaient presque les 1200 milliards de dollars (soit environ $20 \%$ des réserves mondiales) alors que celles du Japon atteignaient environ 800 milliards de dollars (Investir, 2007(a) et Les Echos, 2007). En moyenne, les réserves de change chinoises progressent de 15 milliards chaque mois (Investir, 2007(b)).

${ }^{3}$ L'Asean regroupe les pays suivants : l'Indonésie, la Malaisie, les Philippines, Singapour, la Thaillande, Brunei, le Vietnam, Myanmar (anciennement la Birmanie), le Laos et le Cambodge.

${ }^{4}$ L'Asean +3 regroupe les pays de l'Asean et la Chine, le Japon et la Corée du Sud.
} 
n'existe pas de consensus sur un accord de change régional à l'image du Serpent monétaire puis du Système monétaire européen (SME) dans les années 1970 et 1980 en Europe.

L'objectif de ce papier est d'étudier la nature des chocs macroéconomiques et leur évolution au fil du temps afin de mieux comprendre les choix en terme de régime de change. Pour cela, nous procédons en deux étapes. Dans une première étape, nous examinons le degré de corrélation entre les chocs macroéconomiques à l'aide d'une représentation vectorielle autorégressive structurelle (SVAR par la suite). Cette méthodologie étant sujet à critiques (Boone, 1997, Hénin, 2003 et Fève, 2006), nous utilisons, dans une seconde étape, une approche dynamique basée sur le filtre de Kalman pour étudier l'évolution de la synchronisation des cycles économiques. La seule manière d'adoucir le postulat d'asymétrie structurelle est d'utiliser le filtre de Kalman pour «traiter le sujet de la convergence dynamique des chocs » (Fontagné et Freudenberg, 1999).

Cette méthode fut initialement développée par Haldane et Hall (1991) et Hall et alii (1992). Haldane et Hall (1991) se posent la question relative à la bipolarisation du système monétaire international entre le dollar et le deutsche mark et au rôle de la livre sterling. Ils étudient les liens bilatéraux entre les taux de change de la livre sterling, du dollar et du deutsche mark. Leurs résultats montrent que la livre sterling se détache du dollar pour être davantage liée au deutsche mark. Le renforcement de ce lien s'effectue à partir des années 1980. Hall et alii (1992) analysent la convergence des taux de change, de l'inflation et des taux d'intérêt nominaux à l'aide des tests de cointégration et de la méthode du filtre de Kalman. Leurs résultats montrent l'absence de relations de cointégration en Europe entre 1970 et 1991 par rapport au dollar et au deutsche mark. Par contre, en effectuant une analyse sur deux souspériodes (avant et après 1980), ils trouvent l'existence d'une relation de cointégration des taux de change après 1980. Grâce au filtre de Kalman, ils montrent aussi une convergence qui s'est établie de façon significative au milieu des années 1980.

Boone (1997) utilise cette méthode pour vérifier si les recommandations en terme de convergence des économies ont été (1) mises en place et (2) si elles obtiennent les résultats escomptés : faire que les pays de la zone euro s'approche d'une zone monétaire optimale du point de vue, au moins, de la symétrie des chocs. Utilisant les résultats de Bayoumi et Eichengreen (1996), elle souligne qu'il y a eu une convergence en Europe depuis le début des années 1980. Cette convergence s'est accélérée depuis les années 1990 et le choc de la réunification allemande mais l'union monétaire européenne est depuis constituée d'un cœur et d'une périphérie. 
Babetskii et alii (2005), analysant les PECO, montrent que le processus de convergence des chocs de demande est en cours entre ces pays et l'Union européenne (UE). Pour les chocs d'offre, ces chocs ont tendance à diverger, «fait qui est interprété comme une conséquence des restructurations nécessaires et de l'effet Balassa-Samuelson. Les asymétries des chocs d'offre caractérisent ainsi le processus de rattrapage qui est en cours » (Babetskii et alii, 2005).

Pour la région asiatique, les travaux sont peu nombreux. Cortihnas (2006) et $\mathrm{Xu}$ (2006) effectuent des analyses basées sur le filtre de Kalman.

Cortihnas (2006) utilise un modèle VAR composé du PIB, de l'indice des prix à la consommation et du taux de change effectif réel, puis applique ensuite un modèle espacetemps aux séries de chocs issues de la décomposition ${ }^{5}$ des différents modèles pour quelques pays de l'Asean. Son analyse concerne l'Indonésie, la Malaisie, les Philippines, Singapour et la Thaïlande. A partir de données annuelles allant de 1968 à 2004, il montre une augmentation de la convergence des chocs de demande mais aussi une accélération de la divergence des chocs d'offre depuis les années 1990. Ces résultats sont particulièrement vérifiés pour les Philippines et la Thaïlande qui semblent diverger des autres pays. Par ailleurs, ces résultats ne plaident pas pour la formation d'une union monétaire entre ces pays sans, au préalable, une intégration économique plus forte.

L'étude de Xu (2006) est plus axée sur la Chine et Hong Kong. Xu (2006) analyse la possibilité d'une union monétaire entre la Chine et Hong Kong en prenant la Chine comme pays ancre. Depuis sa rétrocession à la Chine et même si elle bénéficie d'un statut administratif particulier, le sort monétaire de Hong Kong est en jeu. A partir de la démarche de Bayoumi et Eichengreen (1992), il identifie les chocs structurels d'offre et de demande à partir de données trimestrielles de 1988 à 2001. Ses résultats sont des plus intéressants à plusieurs titres. Premièrement, les chocs d'offre et de demande de Hong Kong sont très peu corrélés avec ceux de la Chine mais également avec les États-Unis. Deuxièmement, l'analyse dynamique fait ressortir une très faible convergence, voire une convergence nulle, des chocs de Hong Kong vers la Chine et les États-Unis. De tels résultats sont cruciaux pour l'avenir monétaire de Hong Kong et, à l'heure actuelle, une union monétaire entre la Chine et Hong Kong n'apparaît pas des plus souhaitable.

\footnotetext{
${ }^{5}$ La décomposition utilisée est celle de Clarida et Galì (1994).
} 
Notre étude s'organise de la façon suivante : la section 2 présente l'identification des chocs macroéconomiques à l'aide des modèles SVAR et calcule la corrélation de ces chocs, la section 3 présente une approche dynamique de la convergence et, enfin, la section 4 conclut.

\section{L'identification des chocs par des modèles SVAR}

\section{2. a. La méthode SVAR}

Il existe différentes méthodes pour mesurer la nature des chocs macroéconomiques mais toutes ne sont pas satisfaisantes (Guillaumin, 2007). Parmi toutes ces méthodes, la méthodologie SVAR permet d'identifier l'origine des perturbations (côté offre ou côté demande) mais aussi étudier les réponses à ces perturbations et les délais d'ajustement. Cette approche, initialement développée par Blanchard et Quah (1989), permet d'orthogonaliser les chocs en les décomposant selon leurs effets à long terme. Une décomposition employée précédemment était la décomposition de Cholesky qui ne fait appel à aucune théorie économique. L'inconvénient est que les résultats obtenus dépendent de l'ordre dans lequel sont rangées les séries «la matrice obtenue est triangulaire inférieure; les variables sont rangées de la plus exogène à la plus endogène » (Lardic et Mignon, 2002).

Les travaux de Blanchard et Quah (1989) corrigent cette «anomalie » en imposant des contraintes liées à la théorie économique, ce qui permet d'interpréter chaque choc comme choc d'offre, de demande, de politique monétaire...

Le cadre théorique est celui du modèle offre et demande globales. La référence principale de la modélisation est celle de l'activité et du chômage par Blanchard et Quah (1989). La spécification du SVAR s'effectue en trois étapes.

\section{Estimation}

On estime un VAR sous forme réduite :

$$
X_{t}=\mu+\sum_{i=1}^{p} A_{i} X_{t-i}+u_{t}
$$

Avec $X_{t}=\left(\Delta y_{t}, \Delta p_{t}\right)^{\prime}$ et $u_{t}$ les erreurs de la forme réduite. $y$ est le logarithme du PIB, $p$ représente le logarithme des prix, $\Delta$ indique les variations premières de ces variables et $u_{t}$ est un bruit blanc, de moyenne nulle et de variance $\sigma^{2}$. L'équation (1) peut se réécrire comme :

$$
A(L) X_{t}=\mu+u_{t}
$$

Avec 


$$
A(L)=A_{0}-\sum_{i=1}^{p} A_{i} L^{i} \text { et } A_{0}=I
$$

\section{Inversion du VAR}

De l'équation (2), on peut estimer la forme VMA du processus si $A(L)$ a ses racines extérieures au cercle unité (hypothèse de stationnarité du processus VAR). Ainsi, la forme VMA s'écrit :

$$
X_{t}=c+B(L) u_{t}
$$

Avec $c=A(L)^{-1} \mu$ et $B(L)=A(L)^{-1}$.

On peut réécrire les erreurs de la forme réduite comme une combinaison linéaire, telle que : $u_{t}=S \varepsilon_{t}$ avec $\varepsilon_{t}$ les chocs structurels. C'est-à-dire que l'on considère, comme il est d'usage dans la méthodologie VAR, un vecteur d'innovations normalisées et orthogonales $\varepsilon_{t}=\left(\varepsilon_{t}^{d}, \varepsilon_{t}^{s}\right)^{\prime}$, où $\varepsilon_{t}^{d}$ et $\varepsilon_{t}^{s}$ sont les chocs de demande et d'offre, satisfaisant: $u_{t}=S \varepsilon_{t}$ et $E\left(\varepsilon \varepsilon^{\prime}\right)=I$.

Ce qui implique que $S S^{\prime}=\Sigma$ et que la connaissance d'une matrice d'orthogonalisation $S$ permet d'écrire la représentation VMA en terme de chocs indépendants, dits structurels :

$$
\begin{aligned}
& X_{t}-c=B(L) S \varepsilon_{t} \\
& X_{t}-c=C(L) \varepsilon_{t}
\end{aligned}
$$

Avec

$$
C(L)=B(L) S
$$

En faisant abstraction de la constante $c$, pour des raisons de simplification, on peut réécrire l'équation (5) comme :

$$
X_{t}=B(L)\left[\begin{array}{ll}
s_{y d} & s_{y s} \\
s_{p d} & s_{p s}
\end{array}\right]\left[\begin{array}{c}
\varepsilon_{t}^{d} \\
\varepsilon_{t}^{s}
\end{array}\right]
$$

L'équation (8) peut être transformée, en tenant compte de (7), comme :

$$
X_{t}=\sum_{i=0}^{\infty}\left[\begin{array}{ll}
c_{y d, i} & c_{y s, i} \\
c_{p d, i} & c_{p s, i}
\end{array}\right]\left[\begin{array}{c}
\varepsilon_{t}^{d} \\
\varepsilon_{t}^{s}
\end{array}\right]
$$

Sachant que $u_{t}=S \varepsilon_{t}$, la matrice des chocs structurels que nous devons estimer s'exprime comme $\varepsilon_{t}=S^{-1} u_{t}$. 


\section{Schéma d'identification}

Pour déterminer la forme SVAR, il faut $\frac{n(n+1)}{2}$ restrictions, avec $n$ le nombre de variables du processus VAR. Ici, 3. Dans la matrice des variances-covariances, nous avons 3 coefficients et nous en connaissons 2. Pour estimer le dernier paramètre. il faut une restriction. Cette restriction impose qu'un choc de demande n'ait pas d'effet à long terme sur le PIB.

Ainsi, nous posons la restriction de long terme suivante :

$$
\sum_{i=0}^{\infty} c_{y d, i}=0 \Leftrightarrow C_{y d}(1)=0
$$

Ce type de modèles fut appliqué par un certain nombre d'auteurs. Bayoumi et Eichengreen (1992), par exemple, identifient pour douze pays européens et huit régions américaines, les chocs d'offre et de demande. Puis ils calculent la corrélation, pour chaque type de choc, entre chaque pays européen et l'Allemagne, d'une part, entre chaque région américaine et la région Mid-West, d'autre part ${ }^{6}$.

D'une manière générale, les corrélations obtenues pour les pays européens sont plus faibles que celles obtenues pour les régions américaines. La distinction avant/après SME est peu significative pour les pays européens. Bayoumi et Eichengreen (1992) en concluent que la perte de l'instrument monétaire serait coûteuse pour les pays européens, sauf pour un noyau dur (Belgique, Danemark, France et Pays-Bas) autour de l'Allemagne qui présente un degré de symétrie identique à celui des régions américaines. Il y aurait alors une union monétaire à deux vitesses. Cela étant, ils concluent à des chocs davantage idiosyncratiques entre les pays européens qu'entre les régions américaines ce qui rend difficile la constitution d'une union monétaire.

Chow et Kim (2003) montrent à partir d'un VAR tri-dimensionnel qu'une union monétaire ne serait pas souhaitable dans la zone Asie de l'Est. A partir de données trimestrielles de la production industrielle, ils identifient trois types de chocs : chocs mondiaux, chocs régionaux et chocs domestiques. Les chocs mondiaux sont approximés par les chocs qui touchent les États-Unis et les chocs régionaux sont représentés, selon l'échantillon, européen ou asiatique, par les chocs affectant l'Allemagne ou le Japon. Leur étude se base sur 15 pays européens et 7 pays est-asiatiques (Hong Kong, Indonésie, Corée du Sud, Malaisie, Philippines, Singapour,

\footnotetext{
${ }^{6}$ L'Allemagne et la région Mid-West sont prises comme des ancres, comme le coeur de chacune des deux unions monétaires.
} 
Taïwan). Leur analyse apparaît robuste car ils font des estimations sur des sous-périodes et mettent en place six différents modèles. Dans l'un d'eux, par exemple, ils redéfinissent le choc régional [asiatique] comme la moyenne géométrique des productions industrielles japonaise, coréenne, taïwanaise, malaisienne et singapourienne. Ils concluent qu'une union monétaire dans la zone Asie de l'Est ne serait pas souhaitable. Les économies étudiées seraient davantage assujetties à des chocs macroéconomiques asymétriques (non compensés par d'autres critères des zones monétaires optimales) et qui rendraient dommageables (peutêtre même handicapant) la perte du taux de change comme instrument d'ajustement.

$\mathrm{Ng}$ (2002) trouve des résultats plus contrastés. Son analyse se base sur des données annuelles. Il étudie les pays de l'Asie du Sud-Est. Toujours à partir d'un VAR tri-dimensionnel, trois types de chocs sont identifiés : externe, domestique (propre à l'offre) et domestique (propre à la demande). Ses résultats montrent une corrélation des chocs forte au sein de l'Asean (identifiée à ses cinq pays fondateurs ${ }^{7}$ ) et plus élevée que celle qui prévaut entre les pays européens. L'Indonésie, Singapour et la Malaisie, affichent même un degré de corrélation très élevé. A partir de là et en regardant des critères commerciaux et financiers (inflation, exportations et importations, taux d'intérêt), il affirme qu'une union monétaire serait possible en Asie du Sud-Est constituée de l'Indonésie, de Singapour et de la Malaisie.

Zhang et alii (2004) tentent d'introduire une variable d'ouverture en utilisant un VAR tridimensionnel composé du PIB, de l'indice des prix et du taux de change effectif réel pour identifier les chocs d'offre, de demande et monétaire pour les États-Unis, le Japon, la Corée, Taiwan, la Chine, les Philippines, l'Indonésie, la Malaisie, Singapour, Hong Kong et la Thaïlande. A partir de données trimestrielles sur la période 1980 à 2000, leurs résultats ne plaident pas pour la formation d'une union monétaire entre ces pays. Ils montrent également que les corrélations des trois chocs se sont accentuées après la crise financière et, comme $\mathrm{Ng}$ (2002), qu'une union monétaire locale serait davantage envisageable. Elle serait composée, par exemple, des pays de l'Asean ou les pays nouvellement industrialisés (Hong Kong, Corée, Singapour et Taiwan) car leurs chocs sont assez corrélés et l'ajustement aux chocs est rapide.

\section{2. b. Les résultats pour les pays de l'Asean+3}

Notre étude concerne les pays de l'Asean+3 à quelques exceptions près (annexe 1). Ce choix est fait au vu des accords commerciaux (Asean puis $\mathrm{AFTA}^{8}$ ) et financiers (accords de Chiang

\footnotetext{
${ }^{7}$ Philippines, Indonésie, Malaisie, Singapour et Thaïlande.

${ }^{8}$ L'Asean fut créée en 1967. Un accord signé en 1993 prévoit l'institution d'une zone de libre-échange entre les pays de l'Asean qui devrait être pleinement réalisée en 2003 : l'Asean Free Trade Area (AFTA).
} 
Mai, l'Asian Bond Market Initiative et l'Asian Bond Funds ${ }^{9}$ ) qui peuvent être le support d'un éventuel accord de change ou, tout du moins, d'une meilleure stabilité intra-régionale des taux de change. Ainsi, notre échantillon est composé des pays suivants : Chine, Corée du Sud, Hong Kong, Indonésie, Japon, Malaisie, Philippines, Singapour et Thaïlande. De plus, géographiquement, ces pays sont assez proches.

Les résultats des tests de stationnarité et de cointégration sont présentés dans l'annexe 1. Les données utilisées sont trimestrielles, sur la période 1992.1 à 2003.2. Les sources sont également décrites dans l'annexe 1.

Les tableaux 1 et 2 présentent les corrélations des chocs d'offre et de demande entre les pays de l'Asean+3 obtenues à partir de la décomposition SVAR.

\footnotetext{
${ }^{9}$ L'Asian Bond Market Initiative (ABMI) de l'Asean+3 est destinée à soutenir l'offre d'obligations libellées en monnaies locales par des acteurs privés ou publics tandis que l'Asian Bond Funds de l'EMEAP (Executives'Meeting of East Asia and Pacific) est chargée de soutenir la demande par la création de fonds obligataires en devises étrangères et en monnaies locales alimentées par les pays membres (Guérin et Sa, 2006). Plus récemment, les ministres des Finances des pays de l'Asean +3 «sont tombés d'accord pour un fonds commun de gestion des devises » (Les Echos, 2007). Plus exactement, les réserves cumulées de ces pays étant estimées à quelques 2700 milliards de dollars, cette initiative s'inscrit dans la volonté d'éviter une nouvelle crise financière comme celle de 1997, prolongeant ainsi l'initiative de Chiang Mai (2001).
} 
Tableau 1 : corrélation des chocs structurels d'offre

\begin{tabular}{|c|c|c|c|c|c|c|c|c|c|}
\hline & Chine & Cor & $\mathrm{HK}$ & Ind & Jap & Mal & Phil & Sing & Thaï \\
\hline Chine & $\bar{~} 1$ & $0,61^{\text {**** }}$ & $-0,06$ & $-0,31$ & $-0,25$ & $0,41^{* * *}$ & $0,26^{*}$ & $0,48^{* * *}$ & $-0,14$ \\
\hline Cor & & 1 & 0,00 & 0,04 & $-0,36^{* *}$ & $0,54^{* * *}$ & $0,34^{* *}$ & 0,12 & $-0,30^{*}$ \\
\hline HK & & & 1 & $-0,05$ & 0,07 & $0,52^{* * *}$ & $-0,39$ & 0,23 & 0,15 \\
\hline Ind & & & & 1 & $-0,23$ & $-0,14$ & $0,47^{* * *}$ & $-0,27^{*}$ & $-0,42^{* * *}$ \\
\hline Jap & & & & & 1 & 0,00 & $-0,43^{* * *}$ & 0,18 & $0,26^{*}$ \\
\hline Mal & & & & & & 1 & $-0,04$ & 0,23 & $-0,06$ \\
\hline Phil & & & & & & & 1 & $-0,26^{*}$ & $-0,50^{* * *}$ \\
\hline Sing & & & & & & & & 1 & 0,10 \\
\hline Thaï & & & & & & & & & 1 \\
\hline
\end{tabular}

Cor : Cos seuils de significativité sont de $1 \%(\% *), 5 \%$ (

Cor : Corée du Sud, HK : Hong Kong, Ind : Indonésie, Jap : Japon, Mal : Malaisie, Phil : Philippines, Sing : Singapour,

Thaï : Thaïlande.

Tableau 2 : corrélation des chocs structurels de demande

\begin{tabular}{|c|c|c|c|c|c|c|c|c|c|}
\hline & Chine & Cor & "HK & Ind & Jap & Mal & Phil & Sing & Thaï \\
\hline Chine & 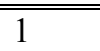 & $-0,17$ & $-0,19$ & $-0,18$ & $-0,02$ & $0,32^{* *}$ & 0,19 & $0,57^{* * *}$ & 0,21 \\
\hline Cor & & 1 & 0,16 & $-0,31$ & 0,08 & $-0,26^{*}$ & $-0,19$ & $-0,33^{* *}$ & $0,25^{*}$ \\
\hline $\mathrm{HK}$ & & & 1 & $-0,02$ & $-0,79^{* * *}$ & 0,04 & $0,35^{* *}$ & $-0,05$ & $0,48^{* * *}$ \\
\hline Ind & & & & 1 & 0,32 & $-0,58^{* *}$ & 0,11 & 0,10 & $-0,42^{*}$ \\
\hline Jap & & & & & 1 & $-0,56^{* * *}$ & $-0,32^{* *}$ & $-0,23$ & $-0,38^{* *}$ \\
\hline Mal & & & & & & 1 & 0,24 & 0,18 & 0,04 \\
\hline Phil & & & & & & & 1 & 0,18 & $0,36^{* *}$ \\
\hline Sing & & & & & & & & 1 & $-0,04$ \\
\hline Thaï & & & & & & & & & 1 \\
\hline
\end{tabular}

Notes : les seuils de significativité sont de $1 \%(* * *), 5 \%(* *)$ et $10 \%(*)$.

Cor : Corée du Sud, HK : Hong Kong, Ind : Indonésie, Jap : Japon, Mal : Malaisie, Phil : Philippines, Sing : Singapour,

Thaï : Thaïlande. 
Les résultats montrent que les chocs de demande sont peu corrélés entre eux. Seulement 14 sur 36 ont des corrélations significatives dont 6 d'une manière positive et 8 de manière négative.

Les chocs d'offre apparaissent légèrement mieux corrélés entre eux. 9 ont des corrélations positives significatives. Seulement 9 ont des corrélations significatives négatives. La Corée, la Malaisie, les Philippines, Singapour et la Thaïlande apparaissent fortement corrélés.

Les chocs de demande du Japon sont, pour la plupart, corrélés significativement à ceux des autres pays, mais d'une manière négative. Les chocs de demande de la Chine sont, eux, très peu significatifs mis à part avec la Malaisie et les Philippines. Ainsi, le rôle de la Chine et du Japon en matière de gestion du taux de change apparaît problématique car ils n'auraient pas intérêt, au vu de ces résultats, à opter pour un régime de change fixe avec coopération.

\section{L'approche dynamique de la convergence}

\section{3. a. Intérêt de la méthode}

Il faut bien remarquer que mesurer la symétrie des chocs grâce au coefficient de corrélation [inconditionnelle] entre les séries de chocs des différents pays représente une manière statique de mesurer les choses. Il est ainsi difficile de distinguer l'évolution des (a)symétries. Il est tout à fait concevable de considérer que les (a)symétries, donc les coefficients de corrélation, évoluent au fil du temps. L'évolution peut éventuellement être mesurée en découpant la période d'étude en plusieurs sous-périodes et calculer les coefficients de corrélation durant ces sous-périodes. Là encore, un problème peut se poser puisque découper la période en plusieurs sous-périodes peut amener à un faible nombre de données par sous-périodes et donc à une non significativité du coefficient de corrélation. Nous pourrions également calculer une corrélation glissante (dans le même esprit que les moyennes mobiles) mais, là aussi, une part d'information serait perdue.

Selon Boone (1997), la corrélation inconditionnelle, même calculée avec une méthodologie SVAR, souffre d'un manque de dynamisme dans l'analyse des chocs. La méthode suppose que les coefficients de corrélation soient stables sur l'ensemble de la période, ce qui dépend de trois hypothèses :

- ces chocs sont structurels, c'est-à-dire reflètent certaines particularités des structures économiques ; 
- il n'y a aucune évolution des similarités structurelles entre les pays (ces similarités sont considérées au début de la période d'estimation et ne connaissent aucune évolution);

- aucun événement extraordinaire n'a perturbé un éventuel phénomène de convergence.

Les relations globales peuvent changer en fonction de la structure de l'économie et des politiques économiques. Les anticipations jouent un rôle important dans beaucoup de relations entre grandeurs globales et les changements de politique altèrent ces anticipations. Par conséquent, les changements de politique peuvent modifier les relations globales (Lucas, 1976). Une des manières de prendre en compte ces changements dans les estimations, donc de calculer la corrélation de ces chocs dans le temps est d'utiliser le filtre de Kalman pour traiter le sujet de la convergence dynamique des chocs.

\section{3. b. La méthode}

La présentation de l'application du filtre de Kalman pour mesurer la convergence s'appuie sur Boone (1997) et Babetskii et alii (2005) ${ }^{10}$.

Supposons $\varepsilon_{t}^{l, i}$ la matrice des chocs de type $l$ du pays $i$ à la date $t$. Deux précisions doivent être apportées. La première concerne le terme $\varepsilon$ qui représente, comme nous l'avons écrit dans la section 2, un choc suite à la décomposition d'un VAR structurel. La seconde est relative au type (l) du choc $\varepsilon$. Ce choc peut être de n'importe quelle nature : d'offre, de demande, monétaire, réelle... Dans notre cas, il s'agit de chocs d'offre ou de demande.

Pour mesurer la convergence dynamique des chocs entre un pays $i$ et un pays $j$, nous pouvons estimer l'équation suivante :

$$
\varepsilon_{t}^{l, i}=\gamma_{t}+\theta_{t} \varepsilon_{t}^{l, j}
$$

Où $\gamma_{t}$ et $\theta_{t}$ sont des coefficients variables ${ }^{11}$ dont les évolutions sont données par les équations d'état suivantes :

$$
\begin{aligned}
& \gamma_{t}=\gamma_{t-1}+\eta_{1, t} \\
& \theta_{t}=\theta_{t-1}+\eta_{2, t}
\end{aligned}
$$

\footnotetext{
${ }^{10}$ La description générale du filtre de Kalman est effectuée en annexe 2.

${ }^{11} \mathrm{Si}$ ces coefficients étaient fixes, l'équation (11) ne serait rien de plus qu'une éventuelle relation de cointégration dont le vecteur cointégrant serait $(1-\theta)$.
} 
La convergence se traduirait, dans l'équation (11), par un coefficient $\gamma=0$ et un coefficient $\theta=1$.

Cependant, une telle mesure de la convergence est particulière puisqu'elle ne mesure la convergence qu'entre les pays $i$ et $j$. Elle ne prend pas en compte un éventuel pays tiers. Boone (1997) propose une mesure de convergence permettant de détecter une convergence plus globale. Cette mesure est davantage appropriée puisque «dans le cadre de l'Union économique et monétaire (UEM), il faut distinguer une convergence européenne, d'un mouvement plus global de convergence. Il faut pouvoir comparer les mouvements d'une variable entre deux pays par rapport aux mouvements de cette variable vis-à-vis du reste du monde $\gg$ (Boone, 1997).

Plus formellement, l'équation de mesure s'écrit alors :

$$
\left(\varepsilon_{t}^{l, j}-\varepsilon_{t}^{l, i}\right)=\alpha_{t}+\beta_{t}\left(\varepsilon_{t}^{l, j}-\varepsilon_{t}^{l, k}\right)
$$

Où $\alpha_{t}$ et $\beta_{t}$ sont des coefficients variables dont les évolutions sont données par les équations d'état suivantes :

$$
\begin{aligned}
& \alpha_{t}=\alpha_{t-1}+\eta_{1, t} \\
& \beta_{t}=\beta_{t-1}+\eta_{2, t}
\end{aligned}
$$

Où $\varepsilon_{t}^{l, m}$ représente la matrice des chocs de type $l$ du pays $m$ (avec $m=i, j, k$ ) à la date $t$.

Nous devons apporter à présent une nouvelle précision. Elle concerne le dilemme concernant les pays de référence, correspondant aux lettres $j$ et $k$ dans l'équation (14). D'après l'équation (14), l'ajout de la matrice $\varepsilon_{t}^{l, k}$ est effectué dans le but de distinguer une convergence entre les pays $i$ et $j$ d'une convergence entre le pays $i$ et un pays tiers (ici $k$ ). En règle générale, ce pays tiers est censé représenter le reste du monde et c'est pourquoi nous choisissons les États-Unis pour représenter le pays $k$ dans l'équation (14).

La plus grande interrogation concerne la lettre $j$. Dans les études précédemment menées concernant notamment l'Union européenne ou la zone euro, le pays $j$ est utilisé comme pays ancre, c'est-à-dire censé constituer le cœur de la zone monétaire. Ainsi, Boone (1997) prend l'Allemagne pour les 11 autres pays qui constituent la zone euro et Babetskii et alii (2005) utilisent l'Union européenne (UE) à 15 pays pour les 10 pays d'Europe centrale et orientale (PECO) intégrant l'UE au $1^{\mathrm{er}}$ mai 2004. 
Dans notre situation ainsi que dans celle de l'étude précédemment menée (Cortihnas, 2006), il est difficile de définir un pays ancre (une force directrice interne) ${ }^{12}$.

Nous pourrions définir une force directrice interne, ce que l'on peut familièrement appeler un poids lourd de la zone car il apparaît peu vraisemblable qu'un pays comme le Japon décide d'ancrer sa monnaie ou sa politique monétaire sur Singapour ou la Malaisie. A la rigueur, ce serait davantage le contraire.

Toutefois, définir un cœur, constitué de un ou plusieurs pays revêt également certaines difficultés notamment le choix des critères censés définir le cœur de la zone : est-ce que nous privilégions un critère commercial, un critère financier, le poids du PIB dans le PIB régional... Nous pourrions choisir comme ancre potentielle la Chine, la Corée et le Japon car les ministres des Finances de ces trois pays ont accepté, en mai 2006, l'idée d'une coopération dans plusieurs domaines y compris celui de la politique de change. L'objectif de ces trois pays est d'utiliser une monnaie commune qui existe déjà avec l'ACU (Asian Currency Unit) pour promouvoir le développement des marchés financiers locaux et la croissance. Lee (2007) se pose la question d'une éventuelle monnaie unique entre la Chine, la Corée et le Japon en utilisant un modèle VAR comprenant le PIB, l'inflation et le taux de change réel par rapport au dollar. Les données utilisées sont trimestrielles et couvrent la période 1980 à 2004. Il utilise l'approche de Bayoumi et Eichengreen (1994) en calculant des coefficients de corrélation statiques puis des corrélations glissantes (dans la lignée des méthodes de moyenne mobile). Ces résultats montrent qu'une monnaie unique ne serait pas envisageable pour ces trois pays sur toute la période, ces trois économies ayant besoin du taux de change comme moyen d'ajustement. Seulement, la crise de 1997 a quelque peu changé la donne et l'analyse post crise, grâce aux corrélations glissantes, montre que cette période serait bien plus enclin à l'adoption d'un système de taux de change fixe entre ces trois économies.

$\mathrm{Au}$ delà de la discussion que nous pouvons avoir sur le choix des variables de cette étude ${ }^{13}$, l'idée d'une monnaie unique entre ces trois pays ne résout pas la question du taux de change pour les autres pays de la région. De plus, les aspects autre qu'économiques doivent être pris en compte (historique, politique, institutionnel).

\footnotetext{
${ }^{12}$ Nous ne citons pas ici l'étude de $\mathrm{Xu}$ (2006) car elle analyse la possibilité d'une union monétaire entre la Chine et Hong Kong en prenant la Chine comme pays ancre. Ce choix apparaît légitime étant donné que Hong Kong fut rétrocédé à la Chine en 1997 et qu'elle est désormais gouvernée comme une région administrative spéciale sous la Basic Law of Hong Kong. Suite à la déclaration sino-britannique du 19 décembre 1984, Hong Kong bénéficie d'un statut administratif spécial pour une durée de 50 ans lui permettant de garder une autonomie relative.

${ }^{13}$ Lee (2007) utilise le taux de change réel par rapport au dollar or il semblerait que le taux de change effectif réel soit plus approprié pour mieux rendre compte de la structure du commerce et de l'impact du taux de change sur cette dernière (Cortihnas, 2006).
} 
C'est pourquoi, pour le moment, l'analyse de la convergence s'effectue sans aucun pays ni zone de références. Ce choix est volontaire mais il pourra, par la suite, évoluer si les économies de l'Asie de l'Est venait à prendre des décisions, notamment monétaires, allant dans ce sens. Ainsi, dans l'équation (14), la lettre $k$ représente le reste du monde, ici approximé par les États-Unis ; la lettre $j$ représente l'ancre potentielle, c'est-à-dire le pays vers lequel les chocs des autres pays, représentés par la lettre $i$, convergent ou non. Nous suivons ainsi Cortihnas (2006) même si son étude ne concernait que quelques pays de l'Asean.

\section{3. c. Les résultats avec l'approche dynamique}

A présent, nous nous servons des séries de chocs estimés dans la section 2 pour mettre en application notre modèle espace-temps afin d'analyser l'évolution des asymétries.

Pour les estimations à partir du modèle espace-temps défini par les équations (14) à (16), l'Indonésie est écartée de l'échantillon à cause du faible nombre de données qui « faussent » l'estimation. De plus, l'échantillon de l'Indonésie est discontinu, ce qui nous pose un problème lors de l'utilisation du filtre de Kalman.

\section{Estimation de $\beta$ dans l'équation (14)}

Pour employer le filtre de Kalman, il faut fixer la valeur initiale de l'équation d'état (équation (16)). Un premier résultat consiste à estimer le paramètre $\beta$ issu de l'équation (14) pour ensuite pouvoir utiliser cette estimation comme valeur de départ du coefficient d'état. Les résultats de cette estimation sont présentés dans les tableaux 3 (chocs de demande) et 4 (chocs d'offre). 
Tableau 3 : estimation du paramètre $\beta$ pour les chocs de demande

\begin{tabular}{rllllllll}
\hline \hline & Chine & Cor & HK & Jap & Mal & Phil & Sing & Thaï \\
\hline \hline Chine & & 0,88 & $1,19^{* *}$ & 0,68 & 0,67 & $0,68^{*}$ & $0,84^{* * *}$ & $1,48^{* * *}$ \\
Cor & $1,10^{* * *}$ & & $0,85^{* * *}$ & $0,69^{* * *}$ & 0,11 & $0,88^{* * *}$ & $1,16^{* * *}$ & 0,56 \\
HK & $1,02^{* * *}$ & 0,42 & & 0,66 & $0,68^{* * *}$ & $0,69^{* * *}$ & $1,00^{* * *}$ & $0,92^{* * *}$ \\
Jap & $0,87^{* * *}$ & $-0,10$ & 0,26 & & 0,19 & $0,66^{* * *}$ & $1,02^{* * *}$ & $1,04^{* * *}$ \\
Mal & $0,93^{* * *}$ & $0,53^{* * *}$ & $0,84^{* * *}$ & $0,69^{* * *}$ & & $0,69^{* * *}$ & $0,98^{* * *}$ & $0,82^{* * *}$ \\
Phil & 0,93 & 0,23 & 0,39 & 0,40 & $-0,28$ & & $0,96^{* * *}$ & $0,75^{* * *}$ \\
Sing & 0,39 & 0,32 & 0,99 & 1,49 & 0,02 & $-0,08$ & & 2,99 \\
Thaï & $1,24^{* * *}$ & 0,50 & $0,69^{* *}$ & 0,74 & 0,39 & 0,68 & $1,03^{* * *}$ & \\
\hline
\end{tabular}

Notes : les seuils de significativité sont de $1 \%(* * *), 5 \%(* *)$ et $10 \%(*)$.

Estimation de $\beta$ à partir de l'équation (13).

Convergence du pays $i$ (horizontal) avec (vers) le pays $j$ (vertical)

Cor : Corée du Sud, HK : Hong Kong, Ind : Indonésie, Jap : Japon, Mal : Malaisie, Phil : Philippines, Sing : Singapour,

Thaï : Thaïlande.

Tableau 4 : estimation du paramètre $\beta$ pour les chocs d'offre

\begin{tabular}{rllllllll}
\hline \hline & Chine & Cor & HK & Jap & Mal & Phil & Sing & Thaï \\
\hline \hline Chine & & 0,17 & $0,47^{* * *}$ & $0,45^{* * *}$ & $-0,01$ & $0,79^{* *}$ & $-0,07$ & 0,03 \\
Cor & 0,46 & & $0,85^{* * *}$ & $0,63^{* * *}$ & $0,38^{* * *}$ & $0,93^{* * *}$ & 0,32 & $0,33^{* * *}$ \\
HK & $0,40^{* * *}$ & 0,51 & & $0,52^{* * *}$ & $0,35^{* * *}$ & $0,62^{* * *}$ & 0,45 & $0,31^{*}$ \\
Jap & 0,60 & 0,48 & $0,96^{* * *}$ & & 0,33 & $0,89^{* * *}$ & 0,23 & 0,48 \\
Mal & 0,17 & 0,14 & $0,51^{* *}$ & 0,43 & & $0,69^{* * *}$ & $-0,06$ & 0,32 \\
Phil & $0,36^{* *}$ & 0,27 & $0,59^{* * *}$ & $0,85^{* * *}$ & $0,52^{* * *}$ & & $-0,05$ & $0,39^{* *}$ \\
Sing & 0,37 & 0,33 & $0,65^{* *}$ & 0,46 & 0,28 & $0,68^{* *}$ & & 0,17 \\
Thaï & $0,23^{* *}$ & $0,49^{* *}$ & $0,50^{* * *}$ & 0,29 & 0,15 & $0,70^{* *}$ & $-0,04$ & \\
\hline \hline
\end{tabular}

Notes : les seuils de significativité sont de $1 \%(* * *), 5 \%(* *)$ et $10 \%\left(^{*}\right)$.

Estimation de $\beta$ à partir de l'équation (13).

Convergence du pays $i$ (horizontal) avec (vers) le pays $j$ (vertical)

Cor : Corée du Sud, HK : Hong Kong, Ind : Indonésie, Jap : Japon, Mal : Malaisie, Phil : Philippines, Sing : Singapour, Thaï : Thailande. 
Une conclusion immédiate est qu'il y aurait une convergence des chocs d'offre vers Singapour, la Corée, la Thaïlande et la Malaisie et, à un degré moindre, le Japon. Il y aurait également une convergence des chocs de demande vers la Corée et la Malaisie et une convergence relative vers le Japon.

Par ailleurs, les coefficients $\beta$ des chocs de demande et d'offre sont plutôt significativement différents de 0 . Sur 56 coefficients, 30 sont significativement différents de 0 pour les chocs de demande et 28 pour les chocs d'offre. L'estimation de ces coefficients montre un chiffre proche de 1 la plupart du temps, signe de chocs relativement asymétriques.

\section{Evolution de $\beta$ dans l'équation (16)}

Le modèle espace-temps décrit par les équations (14) à (16) est estimé par la procédure du filtre de Kalman. Ce modèle va nous permettre d'estimer dans le temps les paramètres $\alpha_{t}$ et $\beta_{t}$

Les estimations dans le temps du paramètre $\beta$ (l'évolution de ce paramètre) nous montre une convergence des chocs de demande (graphiques 1 à 8) :

- de Singapour avec la Malaisie et les Philippines ;

- de la Corée vers la Malaisie.

Elles nous montrent également une convergence des chocs d'offre (graphiques 9 à 16) :

- de la Thaïlande avec le Japon et Singapour ;

- de la Corée avec la Malaisie et la Thaïlande ;

- des Philippines et de la Malaisie avec Singapour.

Suivant notre hypothèse de convergence, $\beta=0$, nous constatons sur les graphiques 1 à 8 (chocs de demande) et 9 à 16 (choc d'offre) qu'il y a plutôt une divergence des chocs, c'est-àdire un coefficient $\beta$ qui tend vers 1 .

Autre constatation, cette divergence semble augmenter après 1997-1998 notamment pour la convergence vers :

- la Malaisie et les Philippines pour les chocs de demande ;

- la Corée, Hong Hong, relativement la Malaisie, les Philippines et Singapour pour les chocs d'offre. 


\section{Choc de demande}

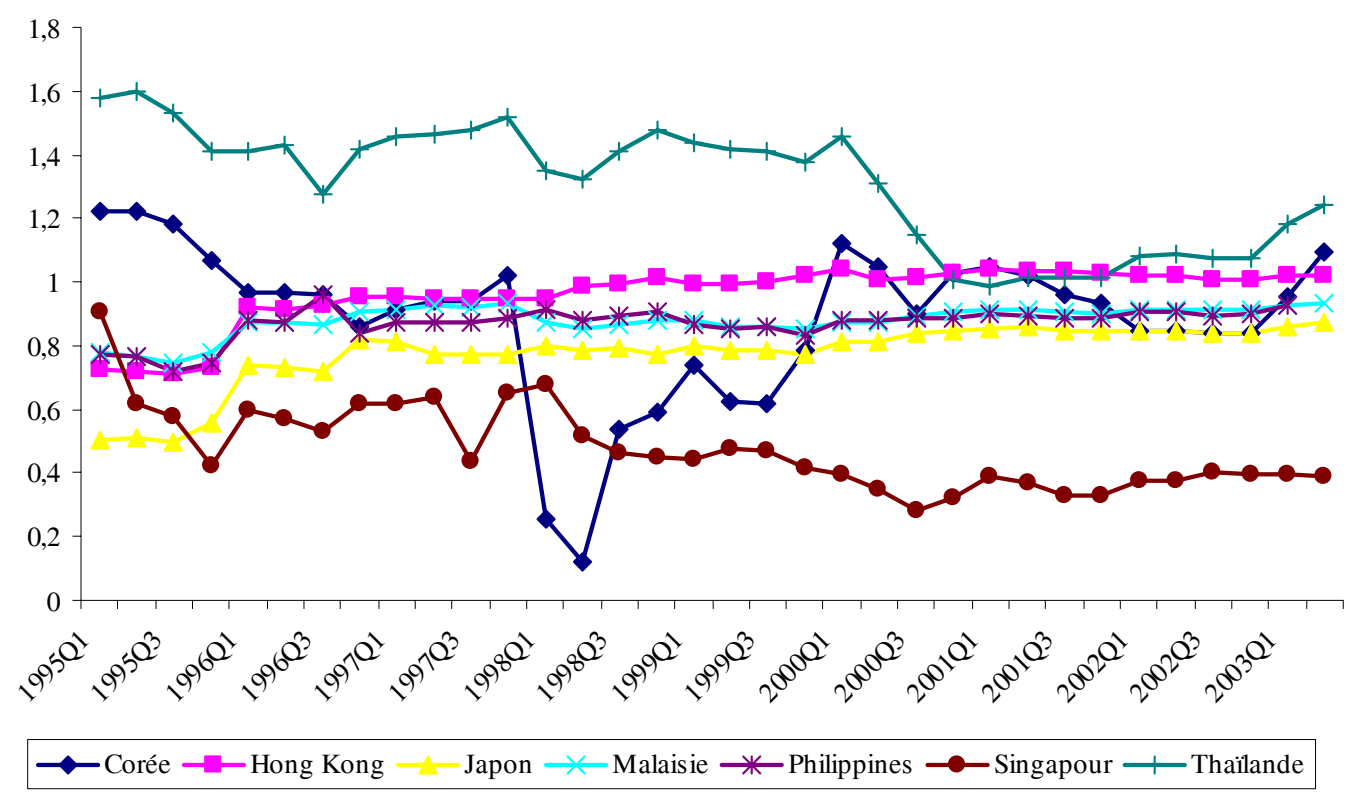

Graphique 1 : évolution de $\beta$ pour la Chine

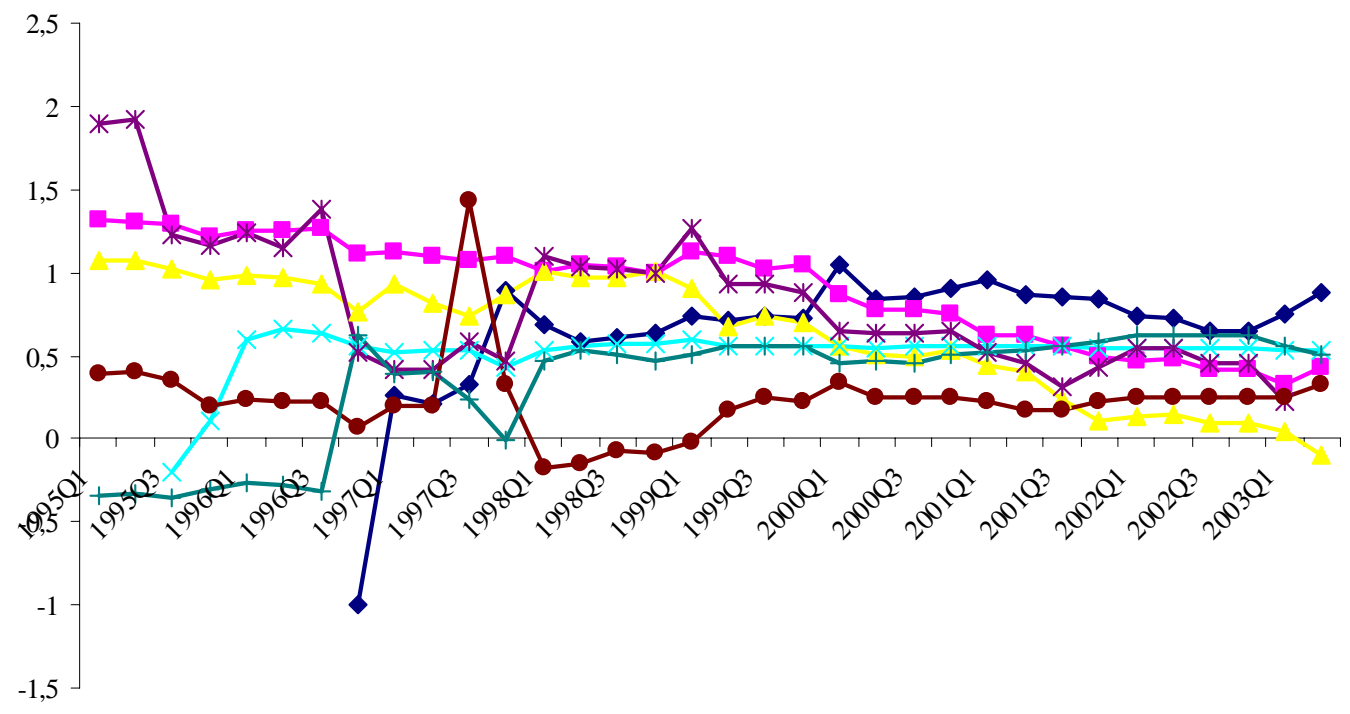

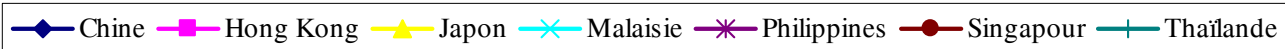

Graphique 2 : évolution de $\beta$ pour la Corée 


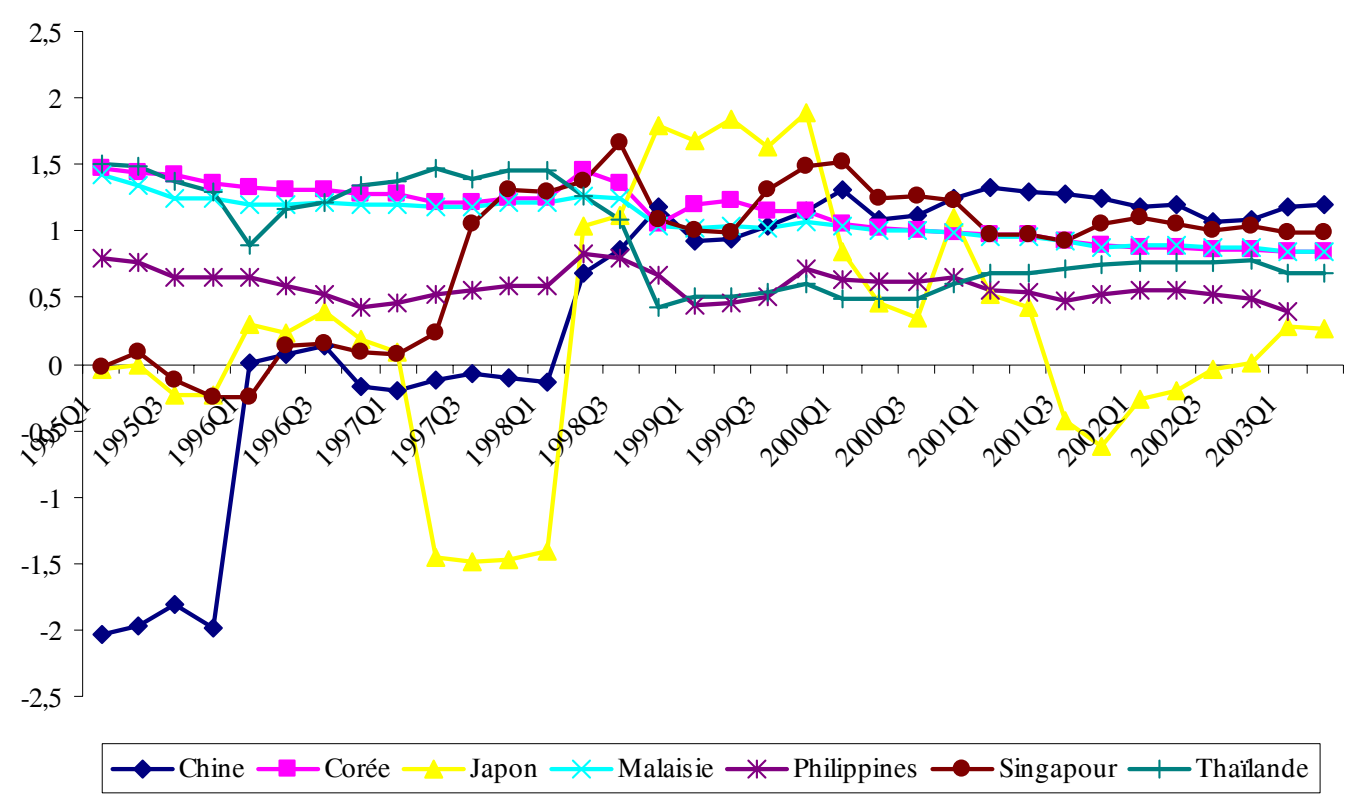

Graphique 3 : évolution de $\beta$ pour Hong Kong

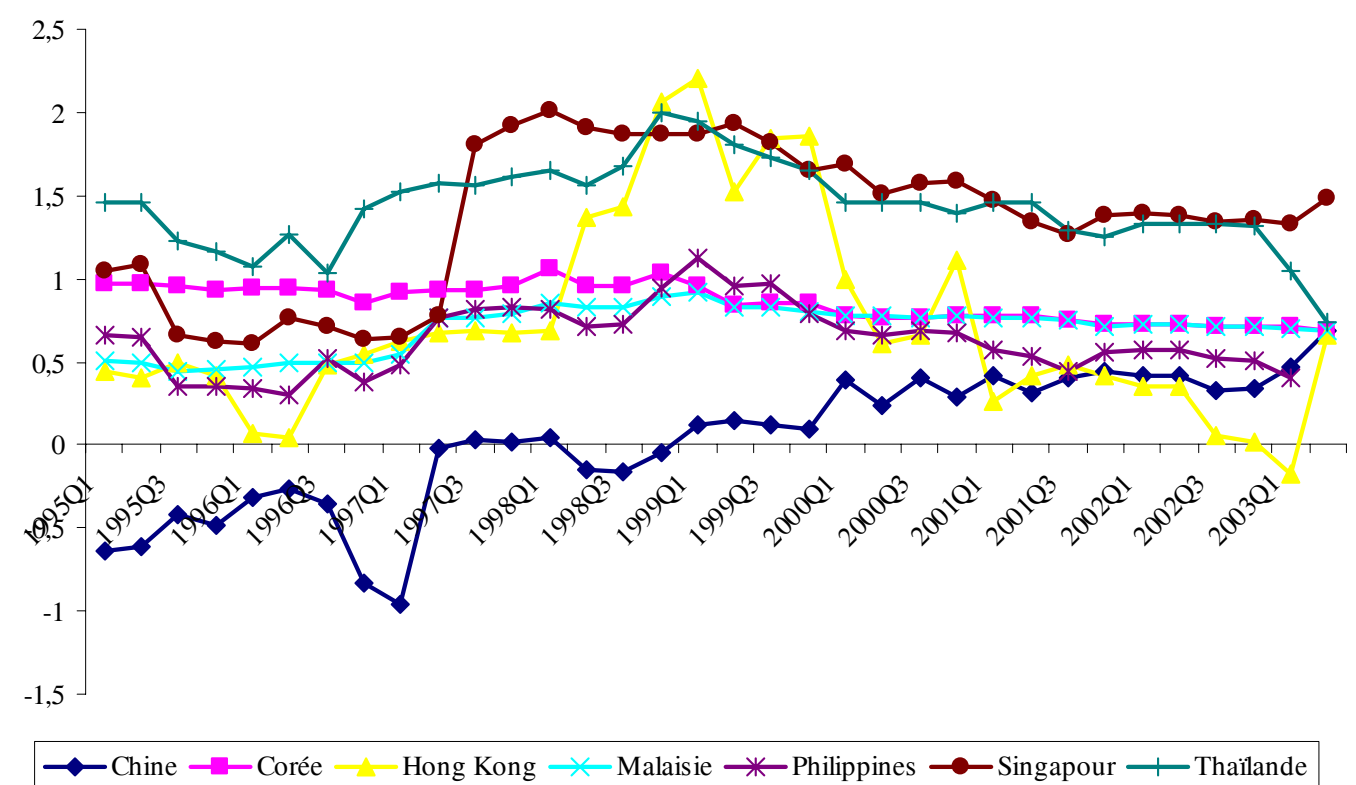

Graphique 4 : évolution de $\beta$ pour le Japon 


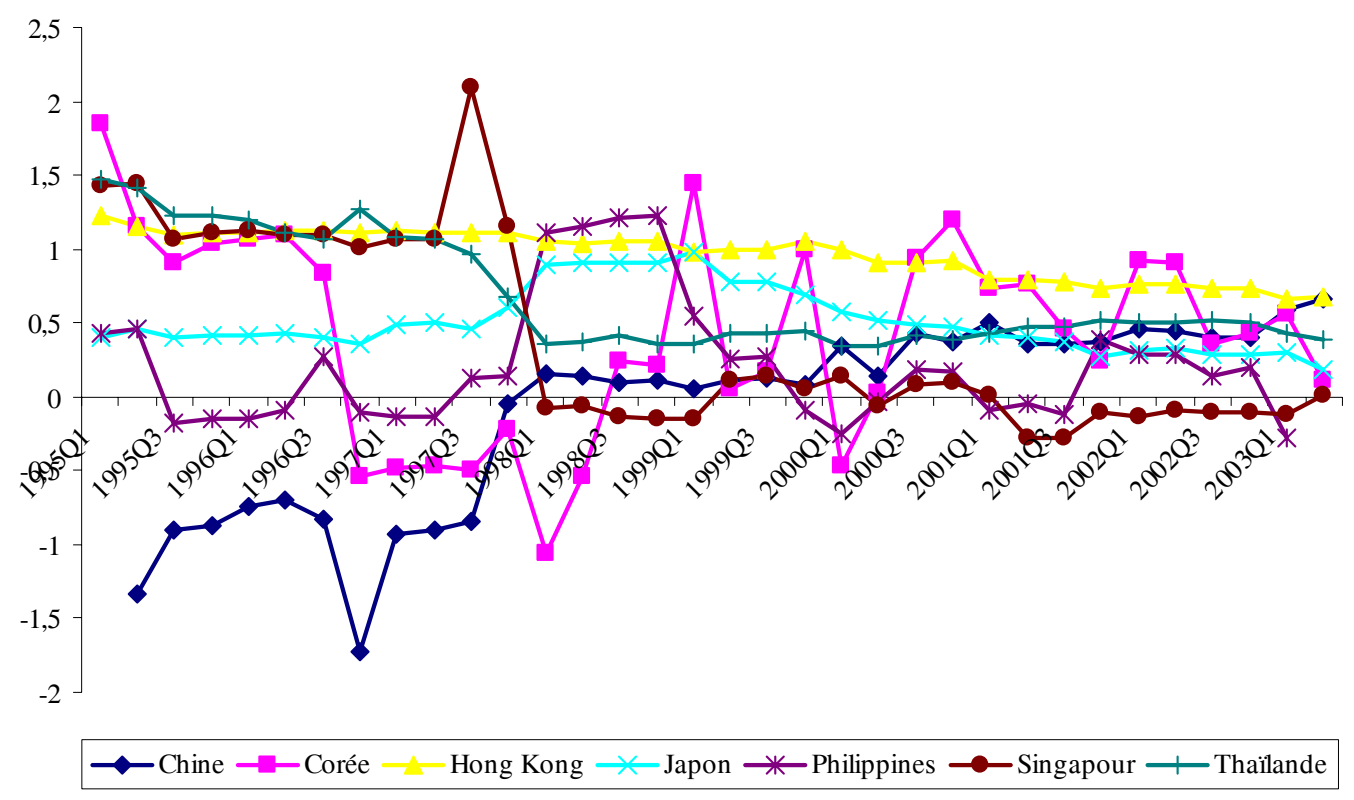

Graphique 5 : évolution de $\beta$ pour la Malaisie

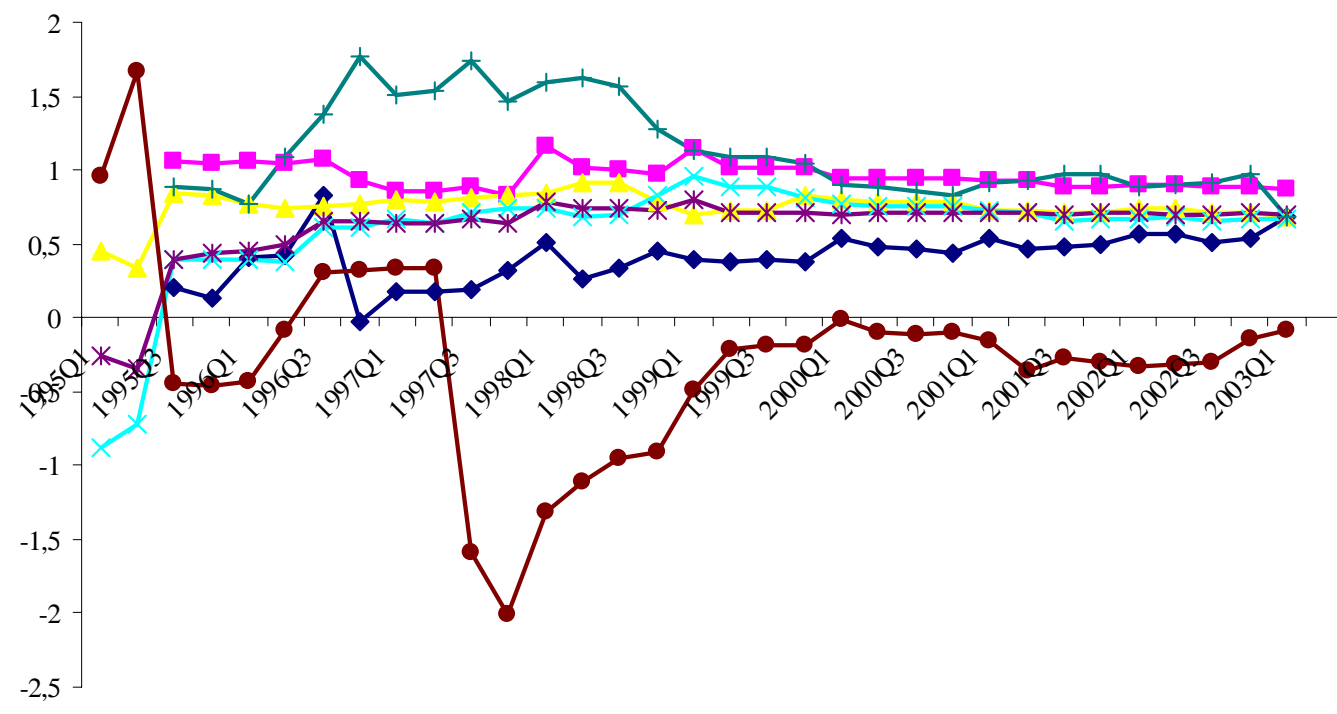

$\neg$ Chine - Corée - Hong Kong $\longleftarrow$ Japon $\multimap$ Malaisie $\longrightarrow$ Singapour $\multimap$ Thaillande

Graphique 6 : évolution de $\beta$ pour les Philippines 


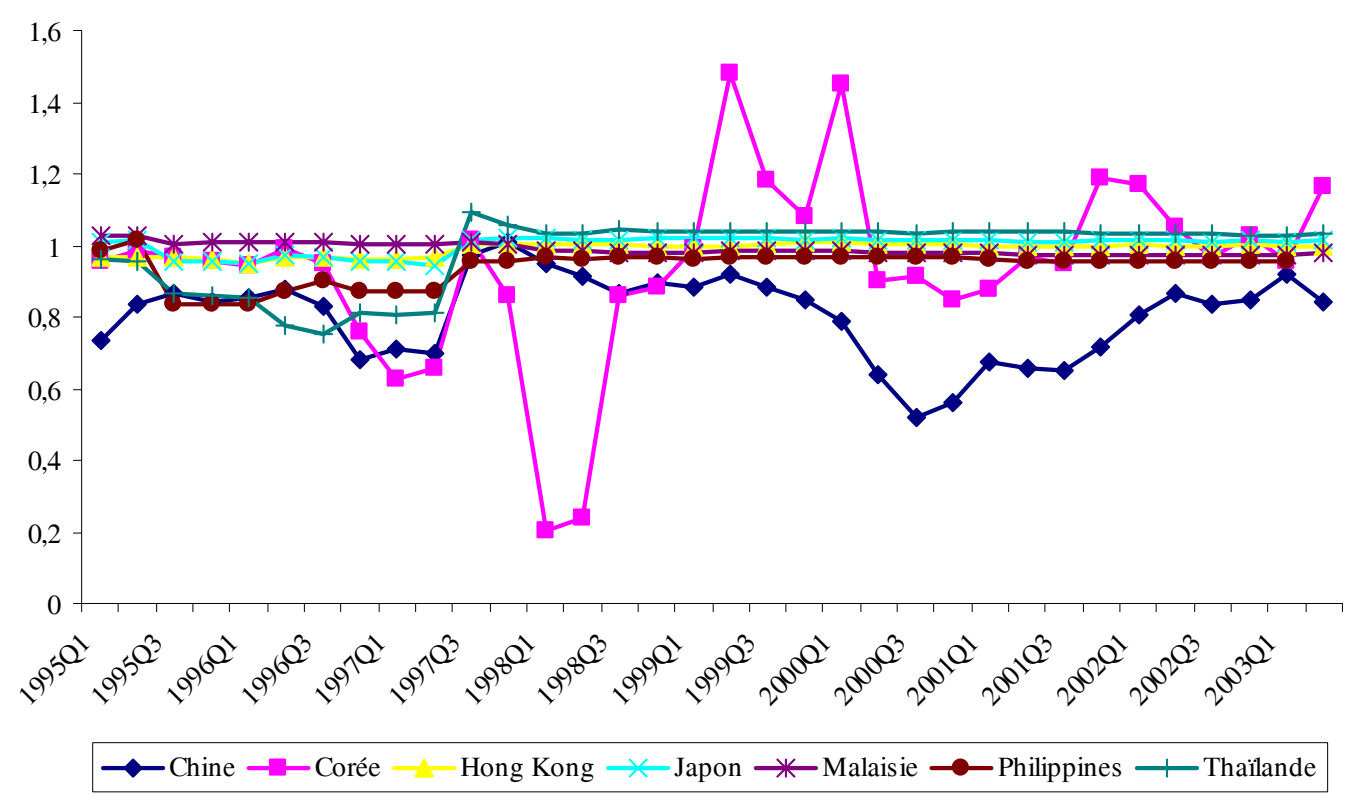

Graphique 7 : évolution de $\beta$ pour Singapour

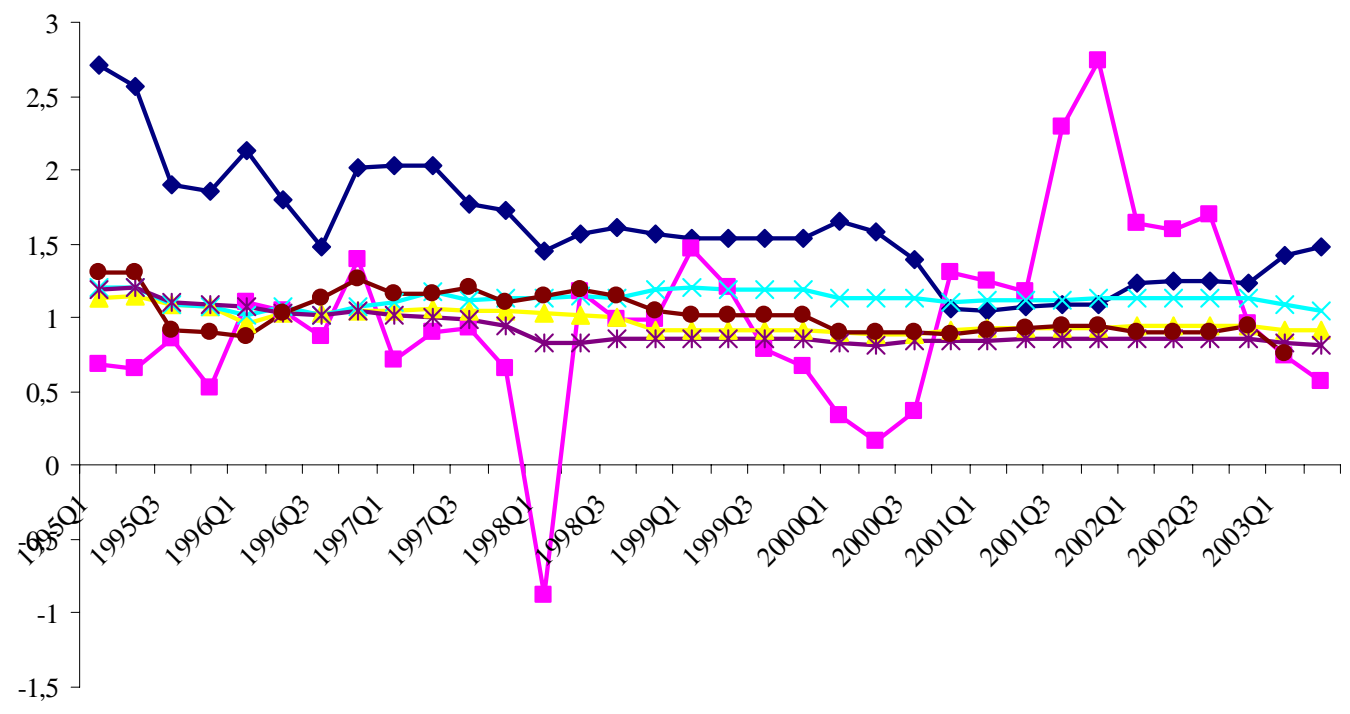

$\neg$ Chine $\multimap$ Corée $\multimap$ Hong Kong $\multimap$ Japon $\multimap$ Malaisie $\multimap$ Philippines

Graphique 8 : évolution de $\beta$ pour la Thaïlande 


\section{Choc d'offre}

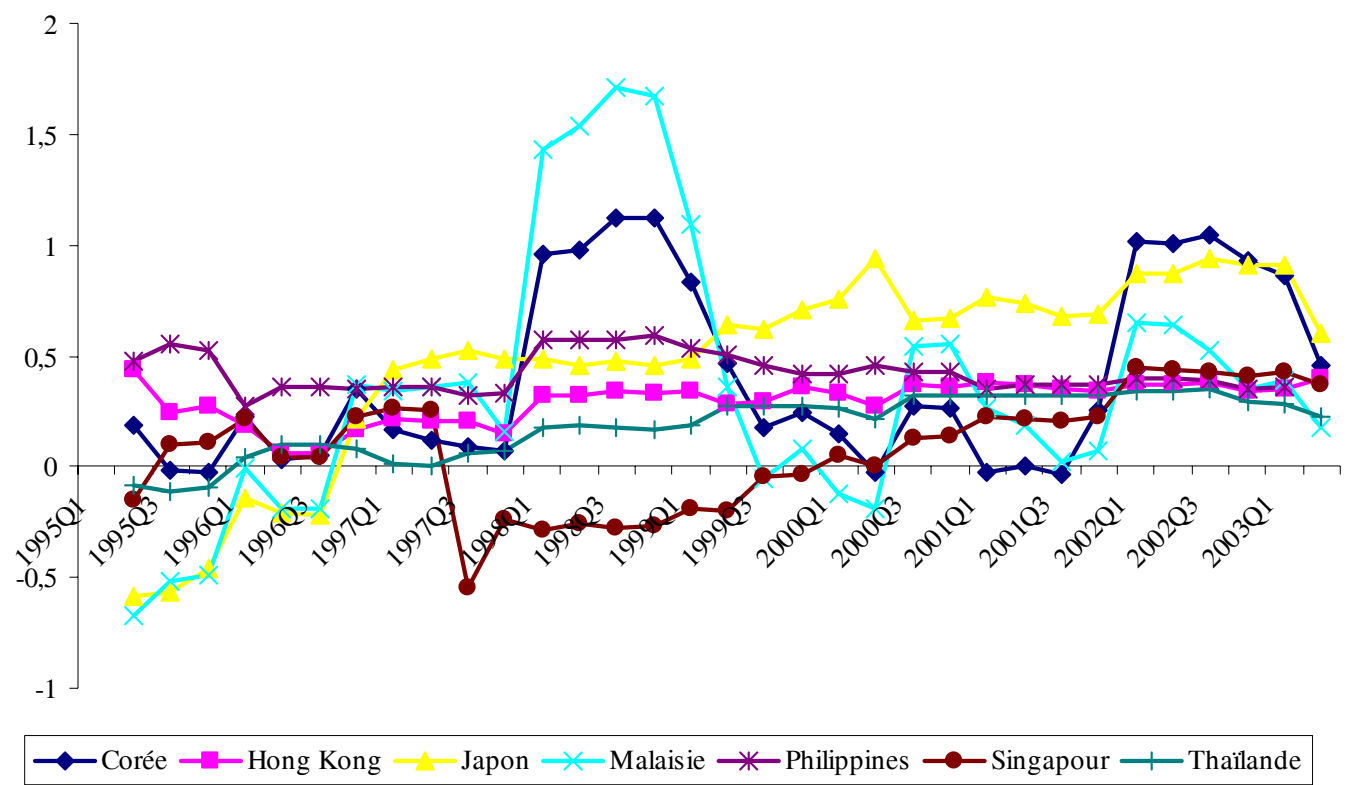

Graphique 9 : évolution de $\beta$ pour la Chine

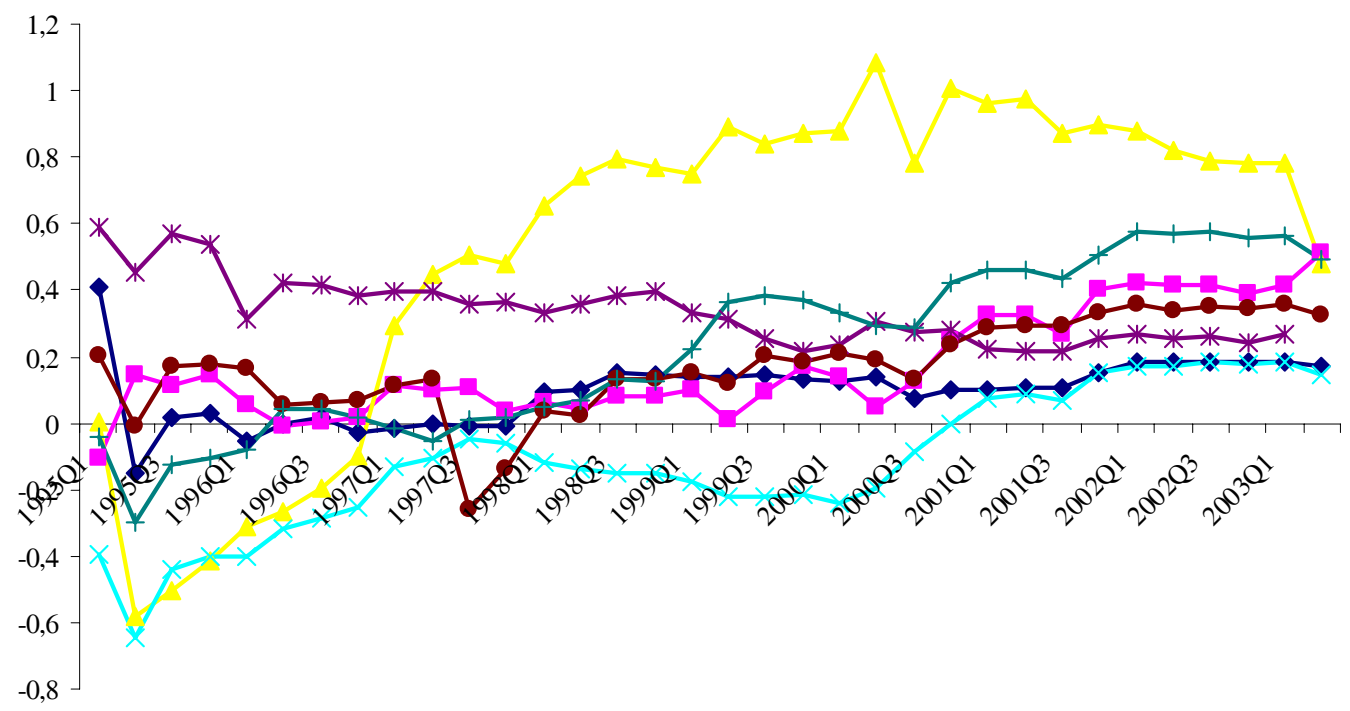

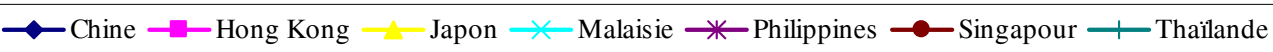

Graphique 10 : évolution de $\beta$ pour la Corée 


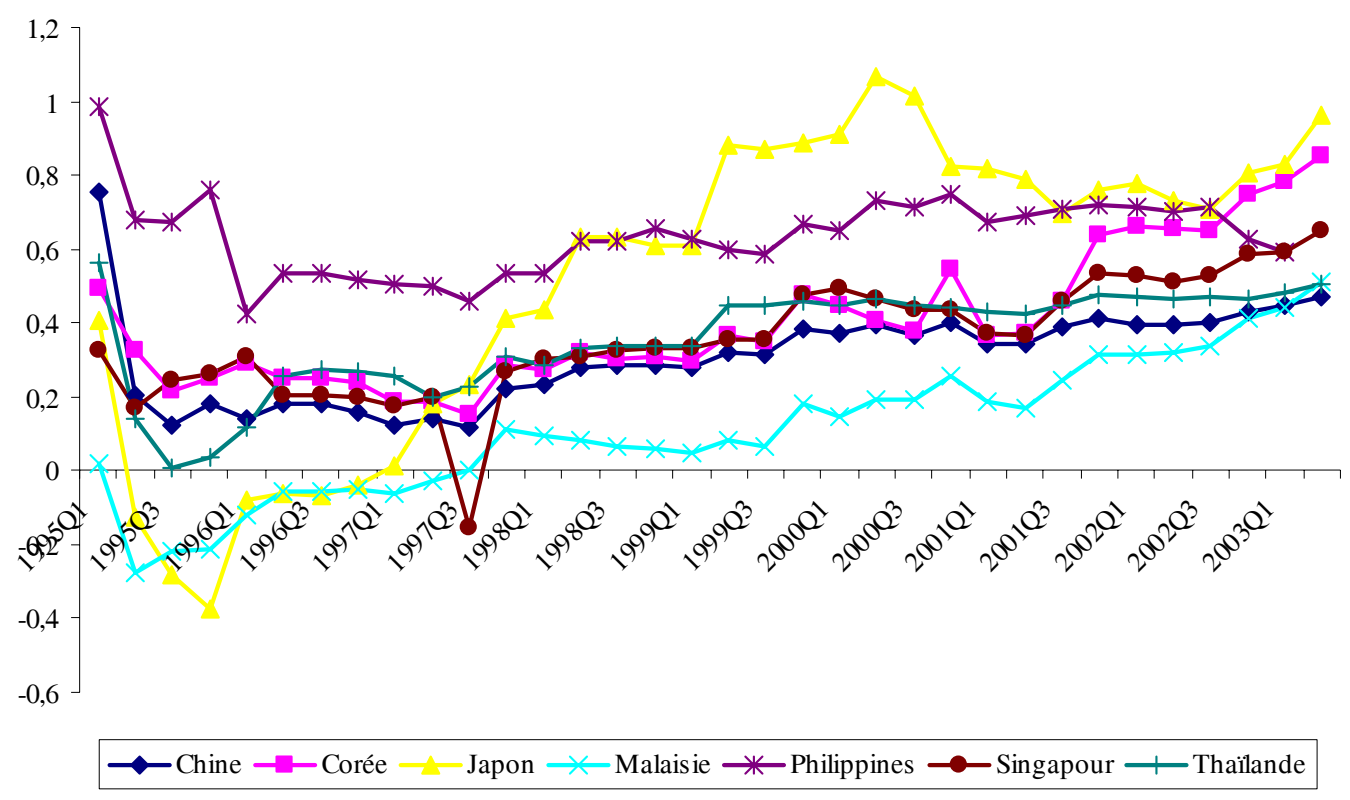

Graphique 11 : évolution de $\beta$ pour Hong Kong

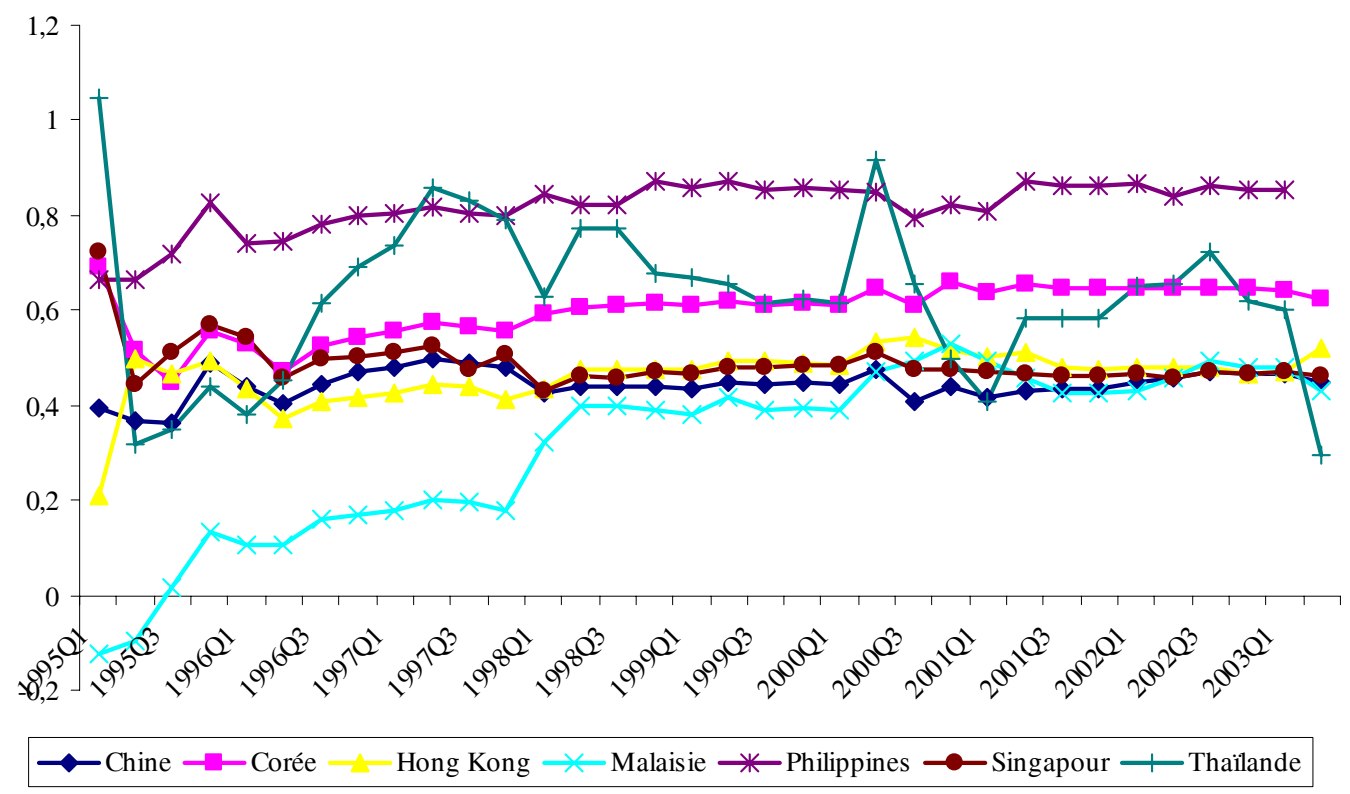

Graphique 12 : évolution de $\beta$ pour le Japon 


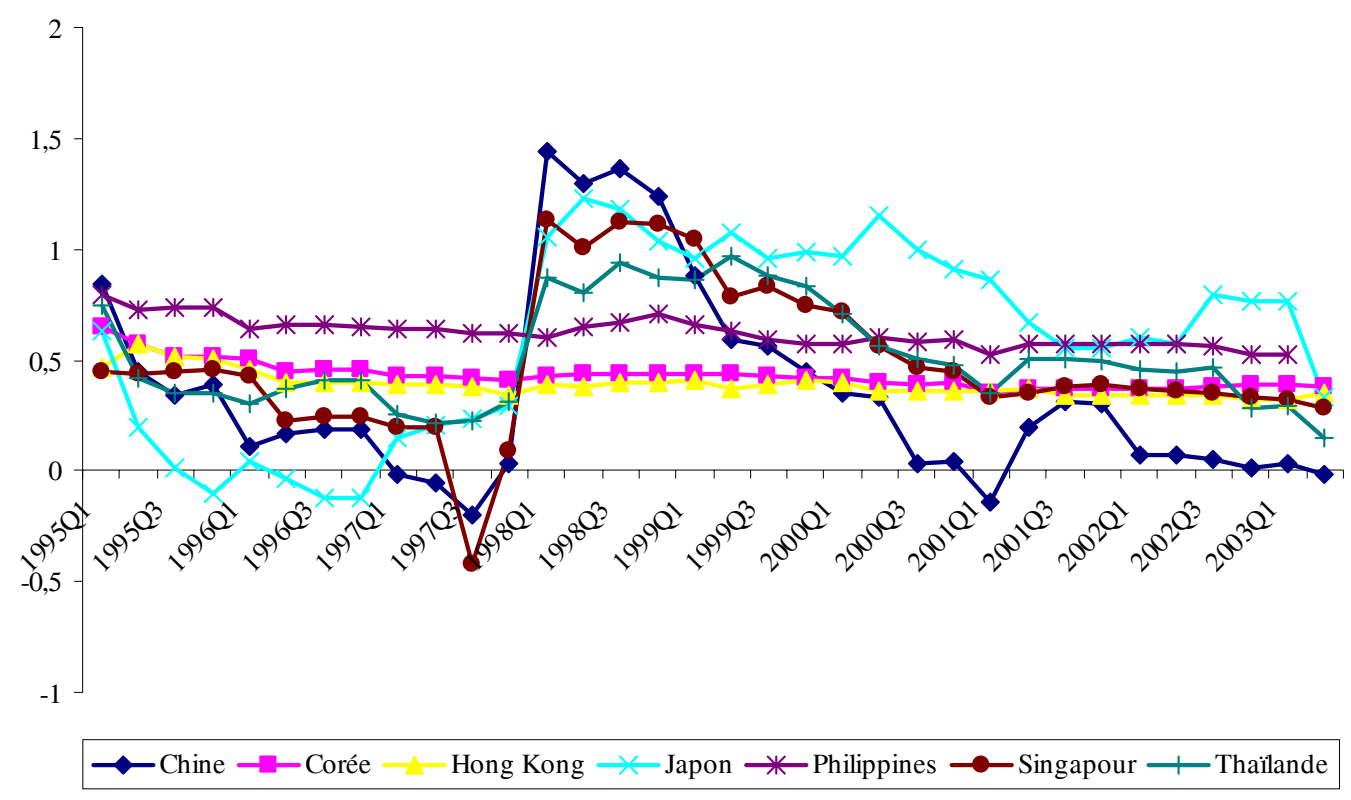

Graphique 13 : évolution de $\beta$ pour la Malaisie

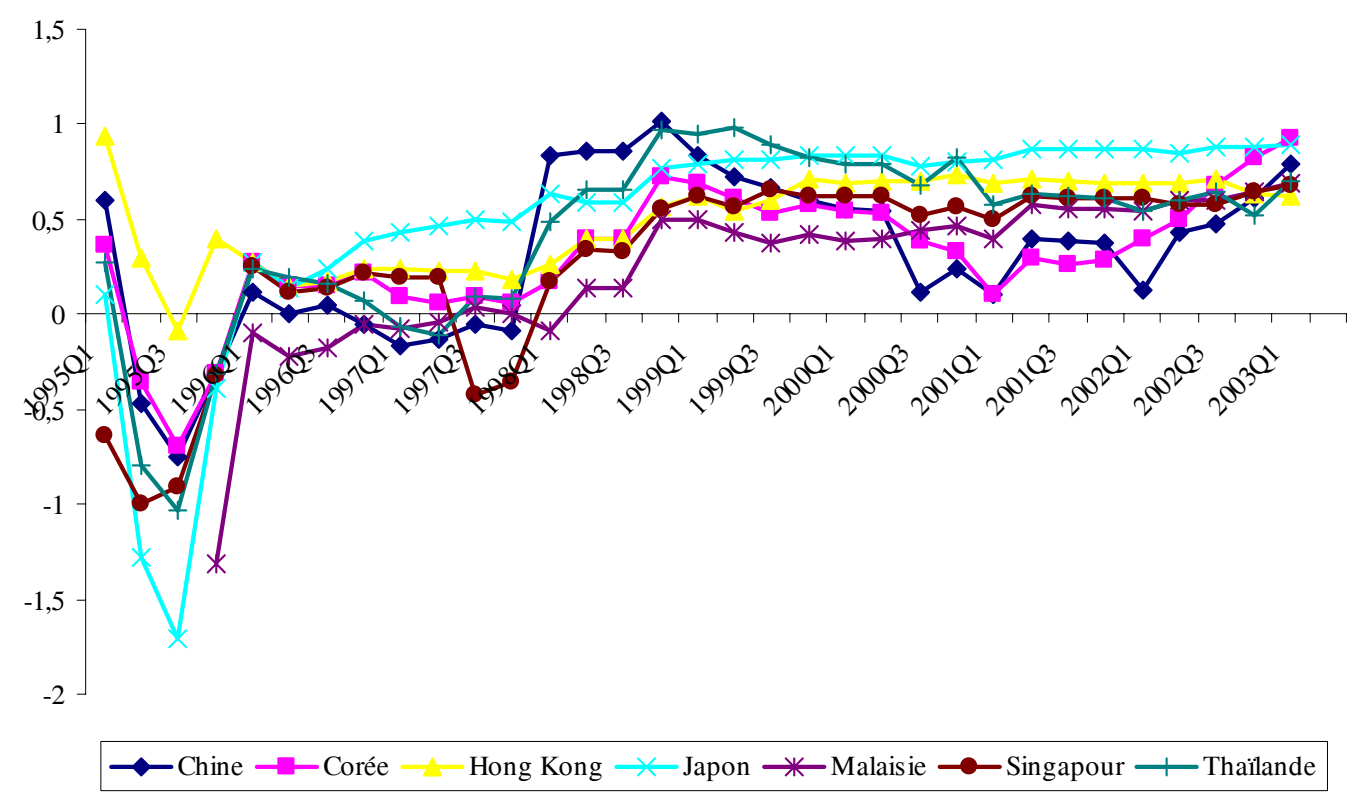

Graphique 14 : évolution de $\beta$ pour les Philippines 


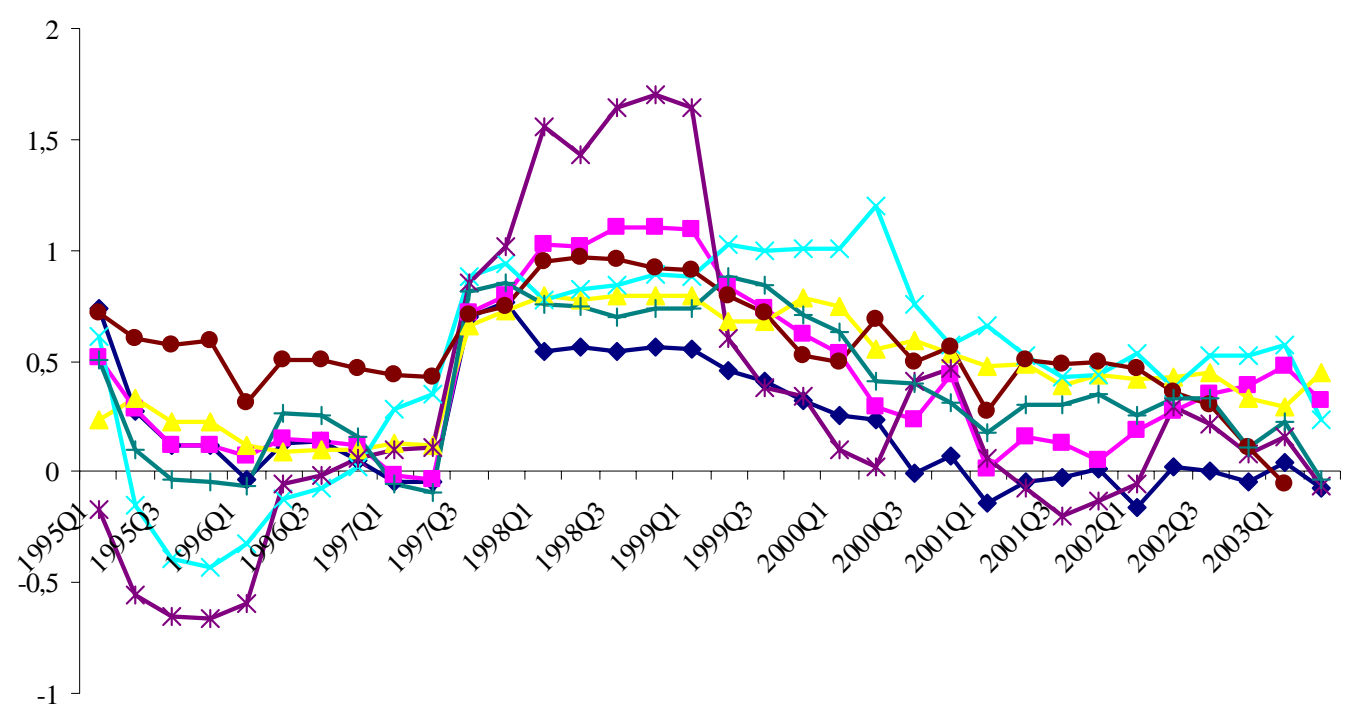

$\neg$ Chine - Corée - Hong Kong $\nsucc$ Japon $\rightarrow$ Malaisie $\longrightarrow$ Philippines $\_$Thailande

Graphique 15 : évolution de $\beta$ pour Singapour

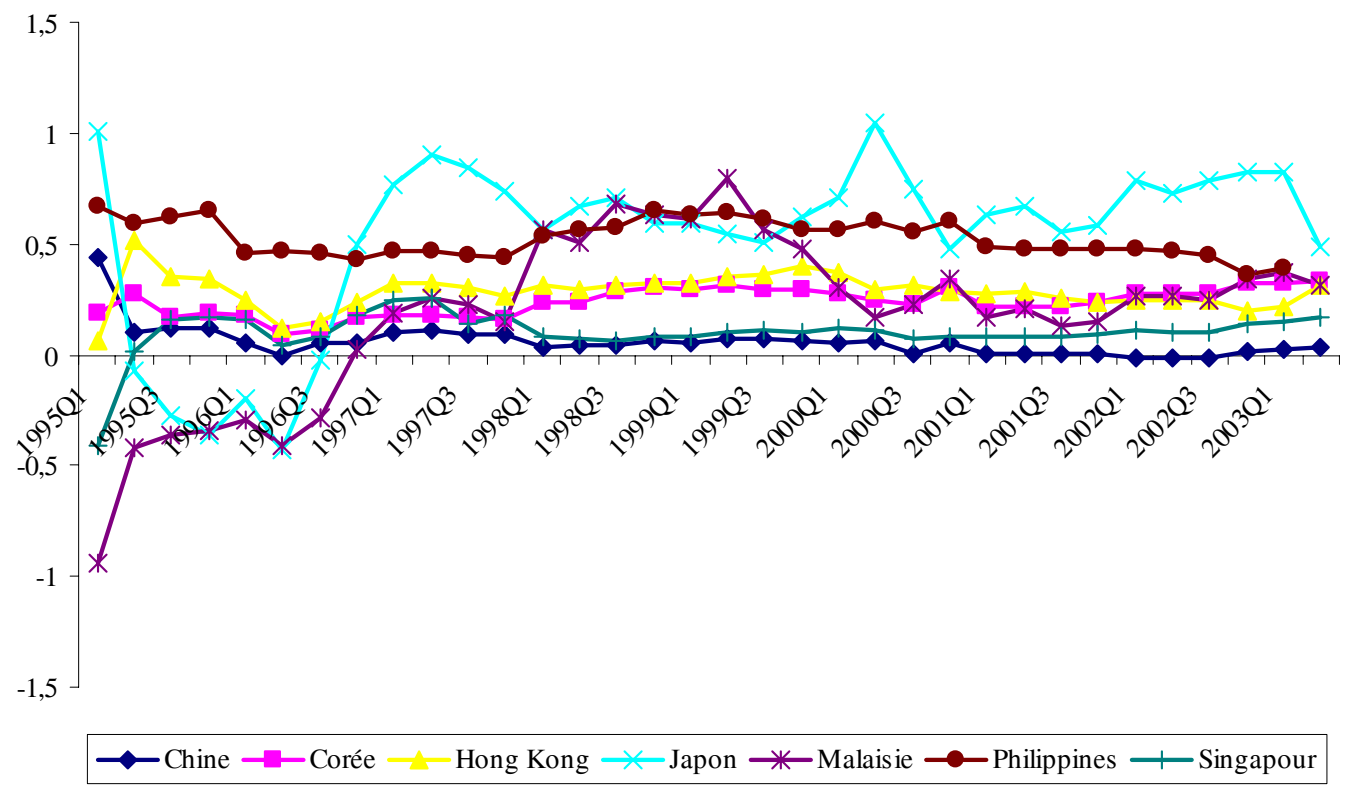

Graphique 16 : évolution de $\beta$ pour la Thaïlande

Pour faciliter et affiner cette analyse graphique, les tableaux 9 à 16 pour les chocs de demande, et 17 à 24 pour les chocs d'offre, détaillés en annexe 3, présentent la moyenne et l'écart-type des coefficients $\alpha$ et $\beta$. Le coefficient $\alpha$ ne doit pas être omis de notre analyse car l'hypothèse de convergence ( $\beta=0)$ est conditionnée au fait que le coefficient $\alpha$ doit être nul. $\alpha$, s'il est correctement estimé, doit également tendre vers 0 puisqu'il représente un bruit blanc et doit ainsi être nul en moyenne. Toutefois, il se peut que $\alpha$ soit différent de 0 . Il peut 
alors exister des questions relatives à la stationnarité du coefficient $\alpha$. L'hypothèse $\alpha=0$, c'est-à-dire où il est un bruit blanc traduit l'hypothèse de stationnarité de ce coefficient. Dans le cas où $\alpha$ ne serait pas stationnaire $(E(\alpha) \neq 0)$, nous pouvons tout de même parler de convergence faible. La faible convergence est définie comme la situation où, simultanément, le coefficient $\alpha$ est différent de 0 mais constant dans le temps, et, dans le même temps, le coefficient $\beta$ tend vers 0 (Babetskii et alii, 2005).

La première ligne de chaque tableau présente les moyennes et les écart-types des coefficients $\alpha$ et $\beta$ pour chaque pays qui diverge ou converge vers un pays en particulier. Les deux dernières lignes constituent des sous périodes que nous distinguons comme avant et après la crise.

Si nous regardons les chocs de demande (tableaux 9 à 16), ils auraient tendance à augmenter vis-à-vis de la Chine, du Japon, de Singapour et de la Thaïlande et à diminuer vis-à-vis de la Corée et de la Malaisie. Hong Kong et les Philippines apparaissent comme des cas particuliers, puisqu'il y a plus de pays dont la convergence augmente par rapport à la période précédente mais dont le coefficient $\beta$ reste élevé.

Par rapport à la moyenne sur la période 1994.1-2003.2, deux situations doivent être distinguées. La première concerne les pays dont les coefficients $\beta$ vis-à-vis d'un pays tiers sont élevés. La seconde, les pays dont les coefficients $\beta$ sont faibles. Pour effectuer la distinction fort/faible, nous choisissons de manière arbitraire le seuil $\beta$ égal à 0,50 .

Pour les chocs de demande, parmi les pays dont les coefficients $\beta$ sont élevés, nous avons la Corée (sauf vis-à-vis de la Thaïlande), Hong Kong, le Japon, la Malaisie (sauf vis-à-vis des Philippines). Pour les pays avec un coefficient $\beta$ faible, nous avons Singapour (sauf vis-à-vis des Philippines et du Japon), les Philippines (sauf vis-à-vis de la Malaisie) et la Thaïlande (sauf vis-à-vis de la Corée). La Chine apparaît entre les deux puisqu'elle a un coefficient $\beta$ faible avec Hong Kong, le Japon, la Malaisie et les Philippines et un coefficient $\beta$ fort avec la Corée, Singapour et la Thaïlande.

Pour les chocs d'offre, les pays avec un coefficient $\beta$ faible sont la Corée (sauf avec le Japon), la Chine, Hong Kong, la Malaisie, Singapour (sauf avec le Japon) et la Thaïlande (sauf avec le Japon et la Malaisie). Les Philippines sont l'unique pays à avoir un coefficient $\beta$ élevé vis-à-vis des pays tiers. Enfin, le Japon est un cas intermédiaire puisqu'il a un coefficient $\beta$ faible avec la Chine, la Corée, Hong Kong et Singapour et fort avec la Malaisie, les Philippines et la Thaïlande. 
Si, à présent, nous effectuons une comparaison des moyennes des coefficients $\beta$ pendant les deux sous-périodes avec celles de la période 1994.1 à 2003.2, nous remarquons, pour les chocs de demande, que seules les Philippines ont un coefficient $\beta$ qui a diminué. La Corée, le Japon ont des coefficients $\beta$ qui ont augmenté. Les cas de Hong Kong, la Malaisie, Singapour et la Chine sont indéterminés puisque certains de leurs coefficients $\beta$ augmentent et d'autres diminuent.

Pour les chocs d'offre, la Chine et les Philippines ont un coefficient $\beta$ qui a diminué durant la période 1999.1-2003.2 alors qu'il augmente par rapport à la moyenne de la période 1994.12003.2 pour la Corée, Hong Kong, le Japon et la Thaïlande. La Malaisie et Singapour sont des cas indéterminés.

Pour résumer, nous pouvons constater deux choses. Premièrement, les chocs macroéconomiques au sein des pays de l'Asean +3 sont relativement asymétriques, il n'y a pas de convergence du paramètre $\beta$ vers 0 , donc il n'y a pas de convergence des chocs entre les pays. Deuxièmement, les coefficients $\beta$ ont tendance à s'écarter de 0 depuis la crise de 1997 . Avant 1997, la relative symétrie des chocs entres les pays de l'Asean+3 pourrait s'expliquer par l'ancrage (de facto ou non) de leur monnaie sur le dollar américain.

Le dollar américain a, sans aucun doute, joué un rôle de vecteur d'intégration entre les pays de l'Asean+3 puisqu'il constituait la monnaie d'ancrage pour la plupart de ces pays. Ceux-ci suivaient ainsi la même politique monétaire que celle des États-Unis et ont eu des cycles qui convergeaient les uns vers les autres (et également vers le cycle américain) grâce au dollar. Le dollar a ainsi joué en Asie le même rôle qu'il a joué en Europe après la Seconde Guerre mondiale, facilitant l'intégration intra-européenne (Mundell, 1973) ${ }^{14}$.

Enfin, nous choisissons de représenter l'évolution de la convergence/divergence entre les pays de la zone étudiée. Les tableaux 5 et 6 présentent ces évolutions pour les chocs d'offre et de demande. Il s'agit de l'augmentation ( $\boldsymbol{\Delta}$ ) ou la diminution $(\boldsymbol{\nabla})$ du coefficient $\beta$ entre la période 1995.1-1998.4 et 1999.1-2003.2. Ces évolutions ont déjà été indiquées dans les tableaux 9 à 24 par ce type de signe.

La véritable convergence (divergence le cas échéant) ne se produit que lorsque les signes $\boldsymbol{\nabla}(\boldsymbol{\Delta} \Delta$ le cas échéant) apparaissent.

\footnotetext{
14 "By keeping their exchange rates fixed to the dollar the European currencies are fixed to one another within exchange rate margins. The monetary integration of the European economies is achieved through the intermediation of the dollar" (Mundell, 1973(a)).
} 
Tableau 5 : convergence ou divergence des pays d'Asie de l'Est entre eux Choc d'offre

\begin{tabular}{|c|c|c|c|c|c|c|c|c|}
\hline $\mathrm{s}$ & Chine & Corée & Hong Kong & Japon & Malaisie & Philippines & Singapour & Thaïlande \\
\hline Chine & - & & & & & & & \\
\hline Corée & $\Delta \boldsymbol{\Delta}$ & - & & & & & & \\
\hline Hong Kong & $\Delta \Delta$ & $\Delta \boldsymbol{\Delta}$ & - & & & & & \\
\hline Japon & $\Delta \Delta$ & $\Delta \boldsymbol{\Delta}$ & $\Delta \boldsymbol{\Delta}$ & - & & & & \\
\hline Malaisie & $\Delta \nabla$ & $\Delta \nabla$ & $\Delta \nabla$ & $\Delta \boldsymbol{\Delta}$ & - & & & \\
\hline Philippines & $\Delta=$ & $\nabla \Delta$ & $\Delta \boldsymbol{\Delta}$ & $\boldsymbol{\Delta} \boldsymbol{\Delta}$ & $\nabla \Delta$ & - & & \\
\hline Singapour & $\Delta \nabla$ & $\Delta \nabla$ & $\Delta \boldsymbol{\Delta}$ & $\nabla \Delta$ & $\Delta \nabla$ & $\Delta \nabla$ & - & \\
\hline Thaïlande & $\Delta \nabla$ & $\Delta \boldsymbol{\Delta}$ & $\Delta \boldsymbol{\Delta}$ & $\nabla \Delta$ & $\Delta \boldsymbol{\Delta}$ & $\Delta \nabla$ & $\Delta \boldsymbol{\Delta}$ & - \\
\hline
\end{tabular}

Notes :

$\boldsymbol{\Delta}$ indique une augmentation du coefficient $\beta$ donc une divergence.

$\boldsymbol{\nabla}$ indique une diminution du coefficient $\beta$ donc une convergence.

Le signe $=$ signifie que le coefficient est identique à celui de la période précédente.

Le premier signe $(\boldsymbol{\Lambda}, \boldsymbol{\nabla})$ signifie l'augmentation, la diminution par rapport à la période précédente du pays $i$ vers le pays $j$.

Le second signe $(\boldsymbol{\Lambda}, \boldsymbol{\nabla})$ signifie l'augmentation, la diminution par rapport à la période précédente du pays $j$ vers le pays $i$. 
Tableau 6 : convergence ou divergence des pays d'Asie de l'Est entre eux

Choc demande

\begin{tabular}{|c|c|c|c|c|c|c|c|c|}
\hline j & Chine & Corée & Hong Kong & Japon & Malaisie & Philippines & Singapour & Thaïlande \\
\hline Chine & - & & & & & & & \\
\hline Corée & $\Delta \nabla$ & - & & & & & & \\
\hline Hong Kong & $\Delta \mathbf{\Delta}$ & $\nabla \nabla$ & - & & & & & \\
\hline Japon & $\Delta \boldsymbol{\Delta}$ & $\nabla \nabla$ & $\Delta \boldsymbol{\Delta}$ & - & & & & \\
\hline Malaisie & $\Delta \boldsymbol{\Delta}$ & $\Delta \boldsymbol{\Delta}$ & $\nabla \nabla$ & $\Delta \nabla$ & - & & & \\
\hline Philippines & $\Delta \mathbf{\Delta}$ & $\nabla \nabla$ & $\nabla \nabla$ & $\Delta \Delta$ & $\nabla \Delta$ & - & & \\
\hline Singapour & $\nabla \nabla$ & $=\boldsymbol{\Lambda}$ & $\Delta \boldsymbol{\Delta}$ & $\Delta \Delta$ & $\nabla \nabla$ & $\nabla \Delta$ & - & \\
\hline Thaïlande & $\nabla \nabla$ & $\Delta \Delta$ & $\nabla \nabla$ & $\nabla \Delta$ & $\nabla \nabla$ & $\nabla \nabla$ & $\Delta \nabla$ & - \\
\hline
\end{tabular}

Notes :

$\boldsymbol{\Delta}$ indique une augmentation du coefficient $\beta$ donc une divergence.

$\boldsymbol{\nabla}$ indique une diminution du coefficient $\beta$ donc une convergence.

Le signe = signifie que le coefficient est identique à celui de la période précédente.

Le premier signe $(\boldsymbol{\Lambda}, \boldsymbol{\nabla})$ signifie l'augmentation, la diminution par rapport à la période précédente du pays $i$ vers le pays $j$.

Le second signe $(\boldsymbol{\Lambda}, \boldsymbol{\nabla})$ signifie l'augmentation, la diminution par rapport à la période précédente du pays $j$ vers le pays $i$. 
Pour les chocs d'offre, il n'existe aucun cas de convergence. Dans les cas où les signes vont dans le même sens, il ne s'agit que de divergence $(\boldsymbol{\Delta} \boldsymbol{\Delta})$.

Pour les chocs de demande, il y a quelques couples de convergence entre :

- la Corée et le Japon, Hong Kong et les Philippines ;

- Hong Kong et les Philippines, la Thaïlande et la Malaisie ;

- la Malaisie et Singapour et la Thaïlande ;

- les Philippines avec la Thaïlande.

Si nous faisons l'hypothèse que ce sont la convergence des chocs de demande qui est le plus important dans une union monétaire plutôt que celle des chocs d'offre, les résultats des tableaux 5 et 6 seraient favorables à une union monétaire dite locale ${ }^{15}$

\section{Conclusion}

Cette étude avait deux objectifs. Le premier était d'introduire une mesure dynamique du degré d'asymétrie des chocs. Pour cela, nous avons utilisé la méthode du filtre de Kalman qui nous a permis de prendre en compte les changements de régime. Ainsi, le problème d'asymétrie structurelle en référence à la critique de Lucas (1976) dont souffre les modèles VAR était surmonté. Le second était d'évaluer à l'aide de cette méthode dynamique l'évolution des chocs auxquels font face les économies d'Asie de l'Est pour mieux comprendre les choix en terme de régime de change.

Nous avons montré que les pays de l'Asie de l'Est présentaient un degré assez élevé d'hétérogénéité aussi bien dans les chocs de demande mais aussi et surtout dans les chocs d'offre et que cette hétérogénéité s'était accentuée au lendemain de la crise financière de 1997. Nos résultats montrent en effet qu'il y a un avant et un après crise. L'avant crise est plutôt marquée par une convergence des chocs qui pourrait s'expliquer, en partie, par l'ancrage, de facto ou non, de ces pays sur le dollar américain. L'après crise marque une divergence des chocs aussi bien de demande que d'offre.

\footnotetext{
${ }^{15}$ L'expression union monétaire locale est reprise par de nombreuses études (Yuen, 2000 ; Ng, 2002 ; Zhang et alii, 2004 ; Guillaumin, 2007). Elle désigne le fait que si une union monétaire devait voir le jour dans la zone Asie de l'Est, elle ne pourrait pas être constituée de l'ensemble des pays de la zone, non seulement pour des raisons économiques mais aussi institutionnelles et politiques, mais davantage par un petit groupe de pays, 3 à 4 maximum, dont les noms peuvent diverger selon les études.
} 
Nous avons également montré qu'il existait une meilleure convergence, relative toutefois, des chocs de demande que d'offre. Ces résultats seraient alors un argument pour des changes fixes du type union monétaire par exemple. Cependant, une union monétaire ne devrait débuter qu'avec quelques pays de l'Asean (Malaisie, Singapour et Thaïlande) dont les relations économiques sont déjà fortes. Cependant, la mise en place d'une telle solution doit faire face à trois problèmes majeurs : la crédibilité du système ${ }^{16}$, la volonté politique et la stabilité du taux de change réel. Par ailleurs, le choix des changes flottants n'est pas forcément meilleurs en cas de chocs asymétriques (Coudert, 2004).

Quatre principales pistes de recherches peuvent être évoquées. En premier, il faut chercher à augmenter le nombre de choc à identifier (Erkel-Rousse, 1997) et essayer de dépasser le cadre initialement proposé par Bayoumi et Eichengreen (1992). Si ce cadre reste pour le moment la référence, il commence à être remis en question (Chari et alii, 2005). Si l'analyse en terme de chocs structurels d'offre et de demande peut apparaître insuffisante, l'identification de davantage de chocs pose quelques problèmes de stabilité et de robustesse, ce qui rend plus délicate l'analyse. Le nombre relativement peu élevé de ce type d'étude pour l'Europe, nous invite à toute la prudence de mise pour l'Asie de l'Est.

En second, la procédure de décomposition de Blanchard et Quah (1989), même si elle est devenue classique, mérite d'être discutée comme le font Cover et alii (2006). La discussion porte sur l'absence de corrélation entre les chocs structurels propre à chaque pays de la zone monétaire étudiée et ceux de la zone monétaire elle-même qui est supposée. Cette source potentielle de biais est déjà évoquée par Blanchard et Quah (1989) puis par Wagonner et Zha (2003) et Bergmann (2005). Par ailleurs, Faust et Leeper (1997) critique vivement l'identification des VAR structurels avec des restrictions de long terme car d'une période à une autre, les effets d'un choc peuvent plus ou moins augmenter en fonction des interactions entre les différentes variables du VAR. Toutefois, Christiano et alii (2005) relativisent ces critiques.

En troisième, si les cadres théorique et économétrique peuvent être améliorés, il faudra continuer à aller au delà des modèles VAR pour corriger la question des asymétries structurelles en utilisant des méthodes dynamiques. Le filtre de Kalman est une première solution. Il faudrait étudier si les modèles de corrélation dynamique conditionnelle issue des

\footnotetext{
${ }^{16}$ La coordination augmente le bien être mais chaque participant a intérêt à dévier pour augmenter ses propres gains.
} 
travaux sur les modèles GARCH multivariés essentiellement appliqués aux séries financières pourraient faire l'objet d'application pour l'étude des chocs macroéconomiques.

Enfin, pour mieux comprendre le choix du régime de change dans ces pays, il serait intéressant et nécessaire d'analyser le degré d'intégration financière et les comportements des politiques monétaires ainsi que leur transmission d'un pays à un autre.

\section{Bibliographie}

Babetskii, I., Boone, L. \& Maurel, M., 2002, « Exchange rate regimes and shocks asymmetry: the case of the accession countries », CEPR discussion paper 3408.

Babetskii, I., Boone, L. \& Maurel, M., 2005, « Exchange rate regimes and shocks asymmetry: the case of the accession countries », Journal of Comparative Economics, vol. 32, $\mathrm{n}^{\circ}$ 2, pp. 212-229.

Bamba, L., 2004, « Analyse du processus de convergence dans la zone UEMOA », Research Paper 2004/18, WIDER.

Bayoumi, T. \& Eichengreen, B., 1992, «Shocking Aspects of European Monetary Unification », NBER working paper 3949.

Bayoumi, T. \& Eichengreen, B., 1996, «Operationalizing the theory of optimum currency areas », CEPR discussion paper 1425.

Bénassy-Quéré, A., 1996, "Régimes et politiques de change en Asie», Economie Internationale, $\mathrm{n}^{\circ}$ 96, pp. 55-79.

Bénassy-Quéré, A. \& Coeuré, B., 2000, «L'avenir des “petites" monnaies : solutions régionales contre solutions en coin », Revue d'Economie Politique, 110 (3), pp. 345-376.

Bénassy-Quéré, A., Coeuré, B., Jacquet, P. \& Pisani-Ferry, J., 2004, Politique économique, De Boeck Université.

Bergmann, M., 2005, « Do long-run restrictions identify supply and demand disturbances ? », working paper, université de Copenhague.

Blanchard, O. \& Quah, D., 1989, « The Dynamics Effects of Aggregate Demand and Supply Disturbances », American Economic Review, vol. 79, $\mathrm{n}^{\circ}$ 4, pp. 655-673.

Boone, L., 1997, «Symétrie des chocs en Union européenne : une analyse dynamique », Economie Internationale, $\mathrm{n}^{\circ}$ 70, pp. 7-34.

Chow, H.-K. \& Kim, Y., 2003, « A Common Currency Peg in East Asia ? Perspectives from Western Europe », Journal of Macroeconomics, vol. 25, n 3, pp. 331-350. 
Christiano, L., Eichenbaum, M. \& Vigfusson, R., 2006, « Assessing structural VARs », NBER working paper 12353.

Clarida, R. \& Galì, J., 1994, « Sources of Real Exchange Rate Fluctuations : How Important are Nominal Shocks? », NBER working paper 4658.

Cortihnas, C., 2006, « Asymmetry of shocks and convergence in selected Asean countries: a dynamic analysis », NIPE WP 3, Universidade do Minho.

Coudert, V., 2004, «Régimes de change et crises: les taux de change fixes sont-ils injustement suspectés ? », in Boyer, R., Dehove, M. \& Plihon, D., Les crises financières, Conseil d'Analyse Economique.

Cover, J., Enders, W. \& Hueng, C., 2006, « Using the aggregate demand-aggregate supply model to identify structural demand-side and supply-side shocks: results using a bivariate VAR », Journal of Money, Credit and Banking, vol. 38, n³, pp. 777-790.

De Grauwe, P., 1999, Economie de l'intégration monétaire, De Boeck Université.

Dubert, M. \& Coudert, V., 2004, «Does exchange rate regime explain differences in economic results for Asian countries », CEPII working paper 2004/05.

Erkel-Rousse, H., 1997, « Degré de flexibilité des marches du travail, ajustement à des chocs asymétriques et union monétaire européenne », Economie et Prévision, 128, pp. 79-100.

Fève, P., 2006, «La modélisation macro-économétrique dynamique », Revue d'Economie Politique, 116 (2), pp. 147-197.

Fontagné, L. \& Freudenberg, M., 1999, «Endogenous Symmetry of Shocks in a Monetary Union », Open Economic Review, 10, pp. 263-287.

Frankel, J., 2000, « No single currency regime is right for all countries or at all times », NBER working paper 7338.

Guérin, J. \& Sa, S., 2006, «Les progress de l'intégration monétaire et financière en Asie », Revue de la stabilité financière, $\mathrm{n}^{\circ}$ 8, pp. 117-135, Banque de France.

Guillaumin, C., 2007, «L'Asie de l'Est remplit-elle les conditions d'une zone monétaire optimale? L'analyse des chocs macroéconomiques », RIEF 2007, soumis à la Revue Economique.

Haldane, A. G. \& S. G. Hall, 1991, «Sterlings'Relationship with the Dollar and the Deutschmark : 1986-1989 », Economic Journal, vol. 101, n 406, pp. 436-443.

Hall, S. G., D. Robertson \& M. Wickens, 1992, Measuring Convergence of the E. C. Economics, The Manchester School, LX Supplement, vol. 60, pp. 99-111.

Hamilton, J., 1994, Times series analysis, Princeton. 
Hénin, P.-Y., 2003, «L'évaluation économétrique des politiques économiques, du "message des innovations" à la "leçon des interventions": une introduction méthodologique », mimeo, CEPREMAP.

Investir, 2007(a), $\mathrm{n}^{\circ}$ 1726, février.

Investir, 2007(b), $\mathrm{n}^{\circ}$ 1742, mai.

Johansen, S., 1988, « Statistical Analysis of Cointegration Vectors », Journal of Economic Dynamics and Control, vol. 12, $\mathrm{n}^{\circ} 2-3$, pp. 231-254.

Kenen, P., 1969, « The Theory of Optimum Currency Areas: an Eclectic View» in Mundell, R. \& Swoboda, A., Monetary Problems of the International Economy.

Lardic, S. \& Mignon, V., 2002, Econométrie des series temporelles macroéconomiques et financières, Economica.

Les Echos, numéros du 23 avril 2007 et du 9 mai 2007.

Lee, K.-S., «A common currency for China, Japan and Korea ?», 24èmes journées internationals d'économie monétaire et bancaire, GDR Européen Monnaie, Banque, Finance, Rennes.

Levy-Yeyati, E. \& Sturzenegger, F., 2005, «Classifying exchange rate regimes: deeds vs. words », European Economic Review, vol. 49, n 6, pp. 1603-1635.

Lucas, R., 1976, «Econometric policy evaluation: a critique », Canergie Rochester Conference Series on Public Policy, pp. 19-46.

McKinnon, R., 1963, « Optimum Currency Areas », American Economic Review, vol. 53, n 4, pp. 717-725.

Mundell, R., 1961, «A Theory of Optimum Currency Areas », American Economic Review, vol. $51, \mathrm{n}^{\circ} 4$, pp. 657-665.

Mundell, R., 1973(a), «Plan for European Currency », in Johnson, H. G. \& Swoboda, A., The Economics of Common Currencies.

Mundell, R., 1973(b), « Uncommon Arguments for Common Currencies », in Johnson, H. G. \& Swoboda, A., The Economics of Common Currencies.

$\mathrm{Ng}, \mathrm{T} ., 2002$, «Should the Southeast Asian Countries form a Currency Union?», The Developing Economies, XL-2, pp. 113-134.

Racicot, F. \& Théorêt, R., 2005, «Quelques applications du filter de Kalman en finance: estimation et prevision de la volatilité stochastique et du rapport cours/bénéfices », cahiers de recherche 15-2005, université du Québec.

Reinhart, C. \& Rogoff, K., «The modern history of exchange rate arrangements : a reinterpretation », NBER working paper 8963. 
Waggonner, D. \& Zha, T., 2003, « Likelihood preserving normalization in multiple equation models », Journal of Econometrics, vol. 114, n 2, pp. 329-347.

Williamson, J., 1999, «The Case for a Common Basket Peg for East Asian Countries », in Collignon, S., Pisani-Ferry, J. \& Park, C. Y., Exchange rate policies in emerging Asian countries, Routledge.

$\mathrm{Xu}, \mathrm{X} ., 2006$, «A currency union for Hong Kong and Mainland China? », Journal of International Money and Finance, vol. 25, n 6, pp. 894-911.

Yuen, H., 2000, «Is Asia an Optimum Currency Area? "Shocking” Aspects of Output Fluctuations in East Asia », national university of Singapore.

Zhang, Z., Sato, K. \& McAleer, M., 2004, «Is a monetary union feasible for East Asia? », Applied Economics, vol. 36, n 10, pp. 1031-1043.

\section{Annexe 1 \\ Description des données et résultats des tests de stationnarité et de cointégration}

Pour les sections 2 et 3, les données utilisées sont trimestrielles, sur la période 1992.1 à 2003.2. Les PIB (la production industrielle le cas échéant) et les indices des prix [à la consommation] sont issus des Statistiques Financières Internationales du Fonds Monétaire International. Pour la Chine, les données pour le PIB sont issues de la base de données Datastream. Les séries de PIB (de production industrielle le cas échéant) sont désaisonnalisées au préalable. L'échantillon comprend les pays d'Asie de l'Est suivants : la Chine, la Corée du Sud, Hong Kong, l'Indonésie, la Malaisie, le Japon, Singapour, les Philippines et la Thaïlande.

Pour des raisons diverses (manque de données, économie en guerre ou en reconstruction, pays qui commence à s'ouvrir au reste du monde), nous éliminons de l'Asean +3 Brunei, le Vietnam, Myanmar, le Laos et le Cambodge.

Avant d'estimer les différents VAR dont nous aurons besoin, nous devons étudier la stationnarité des variables que nous utilisons. Le tableau 7 présente les résultats des tests ADF pour le PIB réel et l'indice des prix à la consommation. 
Tableau 7 : tests ADF de racines unitaires : 1992.1 à 2003.2

PIB réel ${ }^{1}$

Indice des prix

à la consommation

\begin{tabular}{cccccc}
\hline \hline Pays & Niveau & Diff. $1^{\text {ere }}$ & Niveau & Diff. 1 $1^{\text {ere }}$ & Diff. $2^{\text {de }}$ \\
\hline Chine & $\mathrm{I}(1)^{* *}$ & $\mathrm{I}(0)^{* *}$ & $\mathrm{I}(2)^{* *}$ & $\mathrm{I}(1)^{* *}$ & $\mathrm{I}(0)^{* *}$ \\
Corée & $\mathrm{I}(1)^{* *}$ & $\mathrm{I}(0)^{* *}$ & $\mathrm{I}(1)^{* *}$ & $\mathrm{I}(0)^{* *}$ & \\
Hong Kong & $\mathrm{I}(1)^{* *}$ & $\mathrm{I}(0)^{* *}$ & $\mathrm{I}(2)^{* *}$ & $\mathrm{I}(1)^{* *}$ & $\mathrm{I}(0)^{* *}$ \\
Indonésie & $\mathrm{I}(1)^{* *}$ & $\mathrm{I}(0)^{* *}$ & $\mathrm{I}(1)^{* *}$ & $\mathrm{I}(0)^{* *}$ & \\
Japon & $\mathrm{I}(1)^{* *}$ & $\mathrm{I}(0)^{* *}$ & $\mathrm{I}(2)^{* *}$ & $\mathrm{I}(1)^{* *}$ & $\mathrm{I}(0)^{* *}$ \\
Malaisie & $\mathrm{I}(1)^{* *}$ & $\mathrm{I}(0)^{* *}$ & $\mathrm{I}(2)^{* *}$ & $\mathrm{I}(1)^{* *}$ & $\mathrm{I}(0)^{* *}$ \\
Philippines & $\mathrm{I}(1)^{* *}$ & $\mathrm{I}(0)^{*}$ & $\mathrm{I}(1)^{* * *}$ & $\mathrm{I}(0)^{* *}$ & \\
Singapour & $\mathrm{I}(1)^{* *}$ & $\mathrm{I}(0)^{* *}$ & $\mathrm{I}(1)^{* *}$ & $\mathrm{I}(0)^{* *}$ & \\
Thaïlande & $\mathrm{I}(1)^{* *}$ & $\mathrm{I}(0)^{* *}$ & $\mathrm{I}(1)^{* *}$ & $\mathrm{I}(0)^{* *}$ & \\
États-Unis & $\mathrm{I}(1)^{* *}$ & $\mathrm{I}(0)^{* *}$ & $\mathrm{I}(2)^{* *}$ & $\mathrm{I}(1)^{* *}$ & $\mathrm{I}(0)^{* *}$ \\
\hline \hline
\end{tabular}

${ }^{1}$ production industrielle le cas échéant.

Les tests de stationnarité sont menés à partir du logarithme de la variable étudiée.

Diff. $1^{\text {ère }}$ et Diff. $2^{\text {de }}$ sont les différences première et seconde du logarithme de la variable.

$(* * *)$ à $1 \%,(* *)$ à $5 \%$ et $(*)$ à $10 \%$. 
Afin de spécifier le modèle dont nous avons besoin, VAR ou VECM, nous testons, pour chaque pays, l'existence d'une relation de cointégration entre PIB réel et indice des prix [à la consommation] avec l'approche de Johansen (1988). Le tableau 8 présente les résultats :

\begin{tabular}{ccccc}
\hline \hline & Tableau $8:$ test de cointégration : 1992.1 à 2003.2 & \\
\hline \hline Pays & $\lambda_{\text {Trace }}$ & $\lambda_{5 \%}$ & $\lambda_{1 \%}$ & Conclusion \\
\hline Chine & 27,92 & 15,41 & 20,04 & $H_{0}$ acceptée \\
Corée & 16,64 & 15,41 & 20,04 & $H_{0}$ rejetée $^{* * *}$ \\
Hong Kong & 12,59 & 15,41 & 20,04 & $H_{0}$ rejetée $^{* *}$ \\
Indonésie & 16,07 & 15,41 & 20,04 & $H_{0}$ rejetée $^{* * *}$ \\
Japon & 24,56 & 15,41 & 20,04 & $H_{0}$ acceptée \\
Malaisie & 37,39 & 15,41 & 20,04 & $H_{0}$ acceptée \\
Philippines & 12,14 & 15,41 & 20,04 & $H_{0}$ rejetée $^{* *}$ \\
Singapour & 24,14 & 15,41 & 20,04 & $H_{0}$ acceptée \\
Thaïlande & 28,68 & 15,41 & 20,04 & $H_{0}$ acceptée \\
États-Unis & 32,46 & 15,41 & 20,04 & $H_{0}$ acceptée \\
\hline \hline
\end{tabular}

$H_{0}: r=q$ (avec $q$ le nombre de relation de cointégration, ici 1).

$(* * *)$ à $1 \%$ et $(* *)$ à $5 \%$.

\section{Annexe 2}

\section{Description du filtre de Kalman}

La présentation du filtre de Kalman que nous retenons est proche de celles de Hamilton (1994) et Racicot et Théorêt (2005). Cette présentation se déroule en deux étapes : la spécification et l'estimation.

\section{Spécification}

La première spécification concerne l'équation de mesure ou d'observation. Elle décrit une relation entre une variable observée et une variable décrivant l'état de la nature du système :

$$
Y_{t}=A Z_{t}+H X_{t}+\omega_{t}
$$


$\omega_{t}$ est supposé normale, $E\left(\omega_{t}\right)=0$ et $\operatorname{Var}\left(\omega_{t}\right)=R$. Nous observons la variable $X_{t}$ mais pas la dynamique de la trajectoire. $Z_{t}$ est un vecteur de variables exogènes ou prédéterminées, $X_{t}$ est la matrice des observations et $H$ le vecteur d'état des variables inobservables.

La seconde spécification concerne l'équation d'état ou de transition. Cette équation décrit l'évolution des variables inobservables. Elle s'écrit de la manière suivante :

$$
X_{t+1}=F X_{t}+v_{t+1}
$$

$F$ est la matrice de transition et $v$ est la matrice des innovations de l'équation d'état. Ainsi, $E\left(v_{t}\right)=0$ et $\operatorname{Var}\left(v_{t}\right)=Q$.

$\omega_{t}$ et $v_{t}$ sont supposées non corrélées $\left(E\left(w_{t} v_{t}\right)=0\right.$ quel que soit $t$ et $X_{t}$ est non corrélé avec $\omega_{t}$ et indépendant du terme $v_{t}$.

Les équations (17) et (18) représentent un modèle espace-temps que nous pouvons estimer à l'aide du filtre de Kalman.

\section{Estimation}

Pour estimer ce système, nous devons procéder en trois étapes : étape de la prévision, étape de la révision et l'estimation des paramètres.

\section{(i) étape de la prévision}

Nous pouvons calculer la prévision en $t-1$ de $X_{t}$, notée $X_{t / t-1}$, qui est donc l'espérance conditionnelle à toute l'information disponible à la date $t-1$ et la prévision de sa matrice de variance-covariance $P_{t}$, notée $P_{t / t-1}$. Ces prévisions s'écrivent :

$$
\begin{aligned}
& X_{t / t-1}=E\left(F X_{t}\right)=F X_{t-1} \\
& P_{t / t-1}=F_{t} P_{t-1} F_{t}^{\prime}+Q_{t}
\end{aligned}
$$

(ii) étape de la révision

Grâce à l'équation (19), il est possible de prévoir une valeur pour la variable $Y_{t / t-1}$ et de la comparer à la valeur observée $Y_{t}$. La différence entre la valeur observée et la prévision de la valeur constitue l'erreur de prévision. Notons cette erreur $\varepsilon_{t}$. Dès lors :

$$
\varepsilon_{t}=Y_{t}-Y_{t / t-1}=Y_{t}-A Z_{t}-H X_{t / t-1}
$$

Car

$$
Y_{t / t-1}=A Z_{t}+H X_{t / t-1}
$$


La matrice de variance de $\varepsilon_{t}$, notée $G_{t}$, s'écrit :

$$
G_{t}=H_{t}^{\prime} P_{t / t-1} H_{t}+R_{t}
$$

Dès lors, lorsque de nouvelles informations arrivent à la date $t$, il est possible de mettre à jour les équations de prévision (équations (19) et (20)) grâce à $\varepsilon_{t}$ et $G_{t}$. Les équations de mises à jour s'écrivent :

$$
\begin{aligned}
& X_{t}=X_{t / t-1}-\frac{P_{t / t-1} H_{t}^{\prime} H_{t} \varepsilon_{t}}{G_{t}} \\
& P_{t}=P_{t / t-1}-\frac{P_{t / t-1} H_{t}^{\prime} H_{t} P_{t / t-1}}{G_{t}}
\end{aligned}
$$

Le filtre avance ainsi pas à pas et permet d'intégrer l'information dans l'estimation de l'équation de mesure au fur et à mesure qu'elle devient disponible.

(iii) estimation des paramètres

Pour estimer les paramètres, nous avons recours au maximum de vraisemblances :

$$
l=-\frac{1}{2} \sum_{t=1}^{T} \log \left|G_{t}\right|-\frac{1}{2} \sum_{t=1}^{T} v_{t} G_{t}^{-1} v_{t}
$$

\section{Annexe 3}

Convergence des pays de l'Asean+3 ; statistiques des paramètres $\alpha$ et $\beta$ 
Tableau 9: convergence des chocs de demande vers la Chine

\begin{tabular}{|c|c|c|c|c|c|c|c|c|c|c|c|c|c|c|}
\hline \multirow[b]{2}{*}{$94.1-2003.2$} & \multicolumn{2}{|c|}{ Corée } & \multicolumn{2}{|c|}{ HK } & \multicolumn{2}{|c|}{ Jap } & \multicolumn{2}{|c|}{ Mal } & \multicolumn{2}{|c|}{ Phil } & \multicolumn{2}{|c|}{ Sing } & \multicolumn{2}{|c|}{ Thaï } \\
\hline & $\alpha$ & $\beta$ & $\alpha$ & $\beta$ & $\alpha$ & $\beta$ & $\alpha$ & $\beta$ & $\alpha$ & $\beta$ & $\alpha$ & $\beta$ & $\alpha$ & $\beta$ \\
\hline $\begin{array}{l}\text { Moy } \\
\text { ET }\end{array}$ & $\begin{array}{c}0,03 \\
(1,21)\end{array}$ & $\begin{array}{c}0,87 \\
(0,29)\end{array}$ & $\begin{array}{c}0,03 \\
(0,13)\end{array}$ & $\begin{array}{c}0,93 \\
(0,19)\end{array}$ & $\begin{array}{c}0,30 \\
(0,63)\end{array}$ & $\begin{array}{c}0,75 \\
(0,15)\end{array}$ & $\begin{array}{l}-0,04 \\
(0,68)\end{array}$ & $\begin{array}{c}0,85 \\
(0,14)\end{array}$ & $\begin{array}{l}-0,20 \\
(0,29)\end{array}$ & $\begin{array}{c}0,84 \\
(0,15)\end{array}$ & $\begin{array}{c}0,59 \\
(7,68)\end{array}$ & $\begin{array}{c}0,49 \\
(0,42)\end{array}$ & $\begin{array}{c}0,07 \\
(0,74)\end{array}$ & $\begin{array}{c}1,29 \\
(0,25)\end{array}$ \\
\hline $95.1-98.4$ & $\alpha$ & $\beta$ & $\alpha$ & $\beta$ & $\alpha$ & $\beta$ & $\alpha$ & $\beta$ & $\alpha$ & $\beta$ & $\alpha$ & $\beta$ & $\alpha$ & $\beta$ \\
\hline $\begin{array}{l}\text { Moy } \\
\text { ET }\end{array}$ & $\begin{array}{l}-0,65 \\
(1,35)\end{array}$ & $\begin{array}{c}0,86 \\
(0,32)\end{array}$ & $\begin{array}{c}0,04 \\
(0,10)\end{array}$ & $\begin{array}{c}0,90 \\
(0,11)\end{array}$ & $\begin{array}{c}0,09 \\
(0,51)\end{array}$ & $\begin{array}{c}0,71 \\
(0,12)\end{array}$ & $\begin{array}{l}-0,45 \\
(0,65)\end{array}$ & $\begin{array}{c}0,86 \\
(0,06)\end{array}$ & $\begin{array}{l}-0,45 \\
(0,21)\end{array}$ & $\begin{array}{c}0,85 \\
(0,07)\end{array}$ & $\begin{array}{c}0,79 \\
(10,71)\end{array}$ & $\begin{array}{c}0,58 \\
(0,12)\end{array}$ & $\begin{array}{l}-0,04 \\
(0,97)\end{array}$ & $\begin{array}{c}1,45 \\
(0,09)\end{array}$ \\
\hline $99.1-2003.2$ & $\alpha$ & $\beta$ & $\alpha$ & $\beta$ & $\alpha$ & $\beta$ & $\alpha$ & $\beta$ & $\alpha$ & $\beta$ & $\alpha$ & $\beta$ & $\alpha$ & $\beta$ \\
\hline $\begin{array}{c}\text { Moy } \\
\text { ET }\end{array}$ & $\begin{array}{c}0,64 \\
(0,75)\end{array}$ & $\begin{array}{r}0,90 \boldsymbol{\Delta} \\
(0,15)\end{array}$ & $\begin{array}{c}0,06 \\
(0,05)\end{array}$ & $\begin{array}{r}1,02 \mathbf{\Delta} \\
(0,01)\end{array}$ & $\begin{array}{c}0,29 \\
(0,42)\end{array}$ & $\begin{array}{r}0,83 \mathbf{\Delta} \\
(0,03)\end{array}$ & $\begin{array}{c}0,27 \\
(0,54)\end{array}$ & $\begin{array}{r}0,90 \boldsymbol{\Delta} \\
(0,02)\end{array}$ & $\begin{array}{l}-0,01 \\
(0,13)\end{array}$ & $\begin{array}{r}0,89 \boldsymbol{\Delta} \\
(0,02)\end{array}$ & $\begin{array}{c}0,91 \\
(4,91)\end{array}$ & $\begin{array}{r}0,38 \boldsymbol{\nabla} \\
(0,05)\end{array}$ & $\begin{array}{c}0,03 \\
(0,37)\end{array}$ & $\begin{array}{c}1,18 \boldsymbol{\nabla} \\
(0,07)\end{array}$ \\
\hline
\end{tabular}

Source : calculs de l'auteur.

$\boldsymbol{\Delta}$ : augmentation par rapport à la période précédente (divergence)

$\boldsymbol{\nabla}$ : diminution par rapport à la période précédente (convergence).

HK : Hong Kong, Jap : Japon, Mal : Malaisie, Phil : Philippines, Sing : Singapour, Thaï : Thaïlande.

Moy : moyenne ; ET : écart-type. 
Tableau 10 : convergence des chocs de demande vers la Corée du Sud

\begin{tabular}{|c|c|c|c|c|c|c|c|c|c|c|c|c|c|c|}
\hline \multirow[b]{2}{*}{$94.1-2003.2$} & \multicolumn{2}{|c|}{ Chine } & \multicolumn{2}{|c|}{$\mathrm{HK}$} & \multicolumn{2}{|c|}{ Jap } & \multicolumn{2}{|c|}{ Mal } & \multicolumn{2}{|c|}{ Phil } & \multicolumn{2}{|c|}{ Sing } & \multicolumn{2}{|c|}{ Thaï } \\
\hline & $\alpha$ & $\beta$ & $\alpha$ & $\beta$ & $\alpha$ & $\beta$ & $\alpha$ & $\beta$ & $\alpha$ & $\beta$ & $\alpha$ & $\beta$ & $\alpha$ & $\beta$ \\
\hline $\begin{array}{c}\text { Moy } \\
\text { ET }\end{array}$ & $\begin{array}{c}0,01 \\
(0,37)\end{array}$ & $\begin{array}{c}1,22 \\
(1,29)\end{array}$ & $\begin{array}{l}-0,11 \\
(0,16)\end{array}$ & $\begin{array}{c}0,94 \\
(0,33)\end{array}$ & $\begin{array}{c}0,06 \\
(0,17)\end{array}$ & $\begin{array}{c}0,61 \\
(0,37)\end{array}$ & $\begin{array}{l}-0,16 \\
(0,34)\end{array}$ & $\begin{array}{c}0,52 \\
(0,16)\end{array}$ & $\begin{array}{l}-0,22 \\
(0,24)\end{array}$ & $\begin{array}{c}0,85 \\
(0,48)\end{array}$ & $\begin{array}{l}-1,15 \\
(2,74)\end{array}$ & $\begin{array}{c}0,49 \\
(1,50)\end{array}$ & $\begin{array}{c}0,00 \\
(0,50)\end{array}$ & $\begin{array}{c}0,33 \\
(0,37)\end{array}$ \\
\hline $95.1-98.4$ & $\alpha$ & $\beta$ & $\alpha$ & $\beta$ & $\alpha$ & $\beta$ & $\alpha$ & $\beta$ & $\alpha$ & $\beta$ & $\alpha$ & $\beta$ & $\alpha$ & $\beta$ \\
\hline $\begin{array}{c}\text { Moy } \\
\text { ET }\end{array}$ & $\begin{array}{c}0,01 \\
(0,53)\end{array}$ & $\begin{array}{c}1,62 \\
(1,72)\end{array}$ & $\begin{array}{l}-0,13 \\
(0,08)\end{array}$ & $\begin{array}{c}1,16 \\
(0,11)\end{array}$ & $\begin{array}{c}0,06 \\
(0,14)\end{array}$ & $\begin{array}{c}0,94 \\
(0,10)\end{array}$ & $\begin{array}{l}-0,35 \\
(0,19)\end{array}$ & $\begin{array}{c}0,47 \\
(0,23)\end{array}$ & $\begin{array}{l}-0,40 \\
(0,22)\end{array}$ & $\begin{array}{c}1,03 \\
(0,47)\end{array}$ & $\begin{array}{c}-0,54 \\
(1,67)\end{array}$ & $\begin{array}{c}0,23 \\
(0,37)\end{array}$ & $\begin{array}{c}0,21 \\
(0,55)\end{array}$ & $\begin{array}{c}0,09 \\
(0,39)\end{array}$ \\
\hline $99.1-2003.2$ & $\alpha$ & $\beta$ & $\alpha$ & $\beta$ & $\alpha$ & $\beta$ & $\alpha$ & $\beta$ & $\alpha$ & $\beta$ & $\alpha$ & $\beta$ & $\alpha$ & $\beta$ \\
\hline $\begin{array}{c}\text { Moy } \\
\text { ET }\end{array}$ & $\begin{array}{c}0,05 \\
(0,08)\end{array}$ & $\begin{array}{r}0,80 \nabla \nabla \\
(0,11)\end{array}$ & $\begin{array}{c}-0,06 \\
(0,03)\end{array}$ & $\begin{array}{r}0,68 \boldsymbol{\nabla} \\
(0,26)\end{array}$ & $\begin{array}{c}0,00 \\
(0,07)\end{array}$ & $\begin{array}{r}0,37 \boldsymbol{\nabla} \\
(0,29)\end{array}$ & $\begin{array}{c}-0,02 \\
(0,36)\end{array}$ & $\begin{array}{r}0,55 \mathbf{\Delta} \\
(0,01)\end{array}$ & $\begin{array}{c}-0,11 \\
(0,09)\end{array}$ & $\begin{array}{r}0,62 \boldsymbol{\nabla} \\
(0,26)\end{array}$ & $\begin{array}{c}0,16 \\
(0,62)\end{array}$ & $\begin{array}{c}0,23 \\
(0,08)\end{array}$ & $\begin{array}{l}-0,18 \\
(0,13)\end{array}$ & $\begin{array}{r}0,54 \boldsymbol{\Delta} \\
(0,06)\end{array}$ \\
\hline
\end{tabular}

Source : calculs de l'auteur.

$\boldsymbol{\Delta}$ : augmentation par rapport à la période précédente (divergence).

$\boldsymbol{\nabla}$ : diminution par rapport à la période précédente (convergence).

HK : Hong Kong, Jap : Japon, Mal : Malaisie, Phil : Philippines, Sing : Singapour, Thaï : Thaïlande.

Moy : moyenne ; ET : écart-type. 
Tableau 11 : convergence des chocs de demande vers Hong Kong

\begin{tabular}{|c|c|c|c|c|c|c|c|c|c|c|c|c|c|c|}
\hline \multirow[b]{2}{*}{$94.1-2003.2$} & \multicolumn{2}{|c|}{ Chine } & \multicolumn{2}{|c|}{ Corée } & \multicolumn{2}{|c|}{ Jap } & \multicolumn{2}{|c|}{ Mal } & \multicolumn{2}{|c|}{ Phil } & \multicolumn{2}{|c|}{ Sing } & \multicolumn{2}{|c|}{ Thaï } \\
\hline & $\alpha$ & $\beta$ & $\alpha$ & $\beta$ & $\alpha$ & $\beta$ & $\alpha$ & $\beta$ & $\alpha$ & $\beta$ & $\alpha$ & $\beta$ & $\alpha$ & $\beta$ \\
\hline $\begin{array}{c}\text { Moy } \\
\text { ET }\end{array}$ & $\begin{array}{c}0,04 \\
(0,86)\end{array}$ & $\begin{array}{c}0,19 \\
(1,78)\end{array}$ & $\begin{array}{l}-0,10 \\
(0,63)\end{array}$ & $\begin{array}{c}1,25 \\
(0,69)\end{array}$ & $\begin{array}{c}0,18 \\
(0,21)\end{array}$ & $\begin{array}{c}0,40 \\
(1,11)\end{array}$ & $\begin{array}{c}0,00 \\
(0,53)\end{array}$ & $\begin{array}{c}1,00 \\
(1,06)\end{array}$ & $\begin{array}{l}-0,17 \\
(0,27)\end{array}$ & $\begin{array}{c}0,63 \\
(0,18)\end{array}$ & $\begin{array}{l}-1,03 \\
(2,30)\end{array}$ & $\begin{array}{l}-0,39 \\
(5,10)\end{array}$ & $\begin{array}{l}-0,22 \\
(0,60)\end{array}$ & $\begin{array}{c}0,95 \\
(0,36)\end{array}$ \\
\hline $95.1-98.4$ & $\alpha$ & $\beta$ & $\alpha$ & $\beta$ & $\alpha$ & $\beta$ & $\alpha$ & $\beta$ & $\alpha$ & $\beta$ & $\alpha$ & $\beta$ & $\alpha$ & $\beta$ \\
\hline $\begin{array}{l}\text { Moy } \\
\text { ET }\end{array}$ & $\begin{array}{l}-0,23 \\
(0,46)\end{array}$ & $\begin{array}{l}-0,35 \\
(1,03)\end{array}$ & $\begin{array}{l}-0,42 \\
(0,62)\end{array}$ & $\begin{array}{c}1,31 \\
(0,11)\end{array}$ & $\begin{array}{c}0,08 \\
(0,08)\end{array}$ & $\begin{array}{l}-0,07 \\
(0,98)\end{array}$ & $\begin{array}{l}-0,32 \\
(0,48)\end{array}$ & $\begin{array}{c}1,23 \\
(0,08)\end{array}$ & $\begin{array}{l}-0,36 \\
(0,19)\end{array}$ & $\begin{array}{c}0,63 \\
(0,12)\end{array}$ & $\begin{array}{l}-0,70 \\
(1,50)\end{array}$ & $\begin{array}{c}0,50 \\
(0,67)\end{array}$ & $\begin{array}{l}-0,19 \\
(0,74)\end{array}$ & $\begin{array}{c}1,26 \\
(0,28)\end{array}$ \\
\hline $99.1-2003.2$ & $\alpha$ & $\beta$ & $\alpha$ & $\beta$ & $\alpha$ & $\beta$ & $\alpha$ & $\beta$ & $\alpha$ & $\beta$ & $\alpha$ & $\beta$ & $\alpha$ & $\beta$ \\
\hline $\begin{array}{c}\text { Moy } \\
\text { ET } \\
\end{array}$ & $\begin{array}{c}0,03 \\
(0,10) \\
\end{array}$ & $\begin{array}{r}1,16 \boldsymbol{\Delta} \\
(0,12) \\
\end{array}$ & $\begin{array}{c}0,38 \\
(0,35) \\
\end{array}$ & $\begin{array}{r}0,98 \boldsymbol{\nabla} \\
(0,13) \\
\end{array}$ & $\begin{array}{c}0,21 \\
(0,09) \\
\end{array}$ & $\begin{array}{r}0,54 \mathbf{\Delta} \\
(0,79) \\
\end{array}$ & $\begin{array}{c}0,16 \\
(0,43) \\
\end{array}$ & $\begin{array}{r}0,95 \nabla \\
(0,07) \\
\end{array}$ & $\begin{array}{c}0,00 \\
(0,10) \\
\end{array}$ & $\begin{array}{r}0,54 \boldsymbol{\nabla} \\
(0,08) \\
\end{array}$ & $\begin{array}{c}0,35 \\
(0,70) \\
\end{array}$ & $\begin{array}{r}1,12 \mathbf{\Delta} \\
(0,18) \\
\end{array}$ & $\begin{array}{l}-0,11 \\
(0,26) \\
\end{array}$ & $\begin{array}{r}0,64 \nabla \\
(0,11) \\
\end{array}$ \\
\hline
\end{tabular}

Source : calculs de l'auteur.

$\boldsymbol{\Delta}$ : augmentation par rapport à la période précédente (divergence).

$\boldsymbol{\nabla}$ : diminution par rapport à la période précédente (convergence).

Jap : Japon, Mal : Malaisie, Phil : Philippines, Sing : Singapour, Thaï : Thaïlande.

Moy : moyenne ; ET : écart-type. 
Tableau 12 : convergence des chocs de demande vers le Japon

\begin{tabular}{|c|c|c|c|c|c|c|c|c|c|c|c|c|c|c|}
\hline \multirow[b]{2}{*}{$94.1-2003.2$} & \multicolumn{2}{|c|}{ Chine } & \multicolumn{2}{|c|}{ Corée } & \multicolumn{2}{|c|}{$\mathrm{HK}$} & \multicolumn{2}{|c|}{ Mal } & \multicolumn{2}{|c|}{ Phil } & \multicolumn{2}{|c|}{ Sing } & \multicolumn{2}{|c|}{ Thaï } \\
\hline & $\alpha$ & $\beta$ & $\alpha$ & $\beta$ & $\alpha$ & $\beta$ & $\alpha$ & $\beta$ & $\alpha$ & $\beta$ & $\alpha$ & $\beta$ & $\alpha$ & $\beta$ \\
\hline $\begin{array}{c}\text { Moy } \\
\text { ET }\end{array}$ & $\begin{array}{l}-0,36 \\
(0,65)\end{array}$ & $\begin{array}{c}0,04 \\
(0,43)\end{array}$ & $\begin{array}{l}-0,08 \\
(0,49)\end{array}$ & $\begin{array}{c}0,75 \\
(0,37)\end{array}$ & $\begin{array}{l}-0,09 \\
(0,17)\end{array}$ & $\begin{array}{c}0,75 \\
(0,58)\end{array}$ & $\begin{array}{l}-0,07 \\
(0,45)\end{array}$ & $\begin{array}{c}0,60 \\
(0,34)\end{array}$ & $\begin{array}{l}-0,24 \\
(0,30)\end{array}$ & $\begin{array}{c}0,63 \\
(0,22)\end{array}$ & $\begin{array}{l}-0,20 \\
(1,08)\end{array}$ & $\begin{array}{c}1,47 \\
(0,51)\end{array}$ & $\begin{array}{c}-0,08 \\
(1,29)\end{array}$ & $\begin{array}{c}1,34 \\
(0,43)\end{array}$ \\
\hline $95.1-98.4$ & $\alpha$ & $\beta$ & $\alpha$ & $\beta$ & $\alpha$ & $\beta$ & $\alpha$ & $\beta$ & $\alpha$ & $\beta$ & $\alpha$ & $\beta$ & $\alpha$ & $\beta$ \\
\hline $\begin{array}{l}\text { Moy } \\
\text { ET }\end{array}$ & $\begin{array}{l}-0,53 \\
(0,72\end{array}$ & $\begin{array}{l}-0,32 \\
(0,32)\end{array}$ & $\begin{array}{l}-0,35 \\
(0,51)\end{array}$ & $\begin{array}{c}0,95 \\
(0,05)\end{array}$ & $\begin{array}{l}-0,14 \\
(0,09)\end{array}$ & $\begin{array}{c}0,69 \\
(0,52)\end{array}$ & $\begin{array}{l}-0,34 \\
(0,44)\end{array}$ & $\begin{array}{c}0,63 \\
(0,17)\end{array}$ & $\begin{array}{l}-0,48 \\
(0,24)\end{array}$ & $\begin{array}{c}0,60 \\
(0,21)\end{array}$ & $\begin{array}{l}-0,23 \\
(0,94)\end{array}$ & $\begin{array}{c}1,18 \\
(0,59)\end{array}$ & $\begin{array}{l}-0,65 \\
(1,63)\end{array}$ & $\begin{array}{c}1,45 \\
(0,25)\end{array}$ \\
\hline $99.1-2003.2$ & $\alpha$ & $\beta$ & $\alpha$ & $\beta$ & $\alpha$ & $\beta$ & $\alpha$ & $\beta$ & $\alpha$ & $\beta$ & $\alpha$ & $\beta$ & $\alpha$ & $\beta$ \\
\hline $\begin{array}{c}\text { Moy } \\
\text { ET }\end{array}$ & $\begin{array}{c}-0,08 \\
(0,11)\end{array}$ & $\begin{array}{r}0,34 \boldsymbol{\Delta} \\
(0,15)\end{array}$ & $\begin{array}{c}0,25 \\
(0,25)\end{array}$ & $\begin{array}{r}0,77 \boldsymbol{\nabla} \\
(0,07)\end{array}$ & $\begin{array}{c}0,01 \\
(0,03)\end{array}$ & $\begin{array}{r}0,76 \boldsymbol{\Delta} \\
(0,69)\end{array}$ & $\begin{array}{c}0,11 \\
(0,37)\end{array}$ & $\begin{array}{r}0,76 \mathbf{\Delta} \\
(0,06)\end{array}$ & $\begin{array}{c}-0,07 \\
(0,09)\end{array}$ & $\begin{array}{r}0,66 \boldsymbol{\Delta} \\
(0,20)\end{array}$ & $\begin{array}{c}0,40 \\
(0,38)\end{array}$ & $\begin{array}{r}1,52 \mathbf{\Delta} \\
(0,20)\end{array}$ & $\begin{array}{c}0,46 \\
(0,68)\end{array}$ & $\begin{array}{l}1,41 \boldsymbol{\nabla} \\
(0,27)\end{array}$ \\
\hline
\end{tabular}

Source : calculs de l'auteur.

$\boldsymbol{\Delta}$ : augmentation par rapport à la période précédente (divergence).

$\boldsymbol{\nabla}$ : diminution par rapport à la période précédente (convergence).

HK : Hong Kong, Mal : Malaisie, Phil : Philippines, Sing : Singapour, Thaï : Thaïlande.

Moy : moyenne ; ET : écart-type. 
Tableau 13 : convergence des chocs de demande vers la Malaisie

\begin{tabular}{|c|c|c|c|c|c|c|c|c|c|c|c|c|c|c|}
\hline \multirow[b]{2}{*}{$94.1-2003.2$} & \multicolumn{2}{|c|}{ Chine } & \multicolumn{2}{|c|}{ Corée } & \multicolumn{2}{|c|}{$\mathrm{HK}$} & \multicolumn{2}{|c|}{ Jap } & \multicolumn{2}{|c|}{ Phil } & \multicolumn{2}{|c|}{ Sing } & \multicolumn{2}{|c|}{ Thaï } \\
\hline & $\alpha$ & $\beta$ & $\alpha$ & $\beta$ & $\alpha$ & $\beta$ & $\alpha$ & $\beta$ & $\alpha$ & $\beta$ & $\alpha$ & $\beta$ & $\alpha$ & $\beta$ \\
\hline $\begin{array}{c}\text { Moy } \\
\text { ET }\end{array}$ & $\begin{array}{c}0,06 \\
(0,43)\end{array}$ & $\begin{array}{l}-0,22 \\
(0,83)\end{array}$ & $\begin{array}{c}0,16 \\
(0,40)\end{array}$ & $\begin{array}{c}0,51 \\
(0,71)\end{array}$ & $\begin{array}{l}-0,08 \\
(0,12)\end{array}$ & $\begin{array}{c}0,98 \\
(0,22)\end{array}$ & $\begin{array}{c}0,07 \\
(0,12)\end{array}$ & $\begin{array}{c}0,51 \\
(0,21)\end{array}$ & $\begin{array}{l}-0,29 \\
(0,24)\end{array}$ & $\begin{array}{c}0,25 \\
(0,43)\end{array}$ & $\begin{array}{l}-1,54 \\
(2,62)\end{array}$ & $\begin{array}{c}0,30 \\
(1,42)\end{array}$ & $\begin{array}{l}-0,07 \\
(0,41)\end{array}$ & $\begin{array}{r}0,70 \\
(0,38)\end{array}$ \\
\hline $95.1-98.4$ & $\alpha$ & $\beta$ & $\alpha$ & $\beta$ & $\alpha$ & $\beta$ & $\alpha$ & $\beta$ & $\alpha$ & $\beta$ & $\alpha$ & $\beta$ & $\alpha$ & $\beta$ \\
\hline $\begin{array}{l}\text { Moy } \\
\text { ET }\end{array}$ & $\begin{array}{c}0,04 \\
(0,55)\end{array}$ & $\begin{array}{c}-0,77 \\
(0,81)\end{array}$ & $\begin{array}{c}0,10 \\
(0,20)\end{array}$ & $\begin{array}{c}0,29 \\
(0,85)\end{array}$ & $\begin{array}{l}-0,10 \\
(0,06)\end{array}$ & $\begin{array}{c}1,11 \\
(0,05)\end{array}$ & $\begin{array}{c}0,05 \\
(0,12)\end{array}$ & $\begin{array}{c}0,56 \\
(0,21)\end{array}$ & $\begin{array}{l}-0,47 \\
(0,22)\end{array}$ & $\begin{array}{c}0,33 \\
(0,55)\end{array}$ & $\begin{array}{l}-1,18 \\
(1,21)\end{array}$ & $\begin{array}{c}0,90 \\
(0,65)\end{array}$ & $\begin{array}{c}0,02 \\
(0,47)\end{array}$ & $\begin{array}{c}0,96 \\
(0,39)\end{array}$ \\
\hline $99.1-2003.2$ & $\alpha$ & $\beta$ & $\alpha$ & $\beta$ & $\alpha$ & $\beta$ & $\alpha$ & $\beta$ & $\alpha$ & $\beta$ & $\alpha$ & $\beta$ & $\alpha$ & $\beta$ \\
\hline $\begin{array}{c}\text { Moy } \\
\text { ET }\end{array}$ & $\begin{array}{c}0,16 \\
(0,15) \\
\end{array}$ & $\begin{array}{r}0,35 \mathbf{\Delta} \\
(0,17) \\
\end{array}$ & $\begin{array}{c}0,45 \\
(0,28) \\
\end{array}$ & $\begin{array}{r}0,55 \mathbf{\Delta} \\
(0,48) \\
\end{array}$ & $\begin{array}{c}0,00 \\
(0,04) \\
\end{array}$ & $\begin{array}{r}0,84 \boldsymbol{\nabla} \\
(0,12) \\
\end{array}$ & $\begin{array}{c}0,11 \\
(0,08) \\
\end{array}$ & $\begin{array}{r}0,47 \boldsymbol{\nabla} \\
(0,22) \\
\end{array}$ & $\begin{array}{l}-0,11 \\
(0,06) \\
\end{array}$ & $\begin{array}{r}0,11 \boldsymbol{\nabla} \\
(0,23) \\
\end{array}$ & $\begin{array}{l}-0,06 \\
(0,32) \\
\end{array}$ & $\begin{array}{r}-0,04 \nabla \\
(0,13) \\
\end{array}$ & $\begin{array}{l}-0,10 \\
(0,10) \\
\end{array}$ & $\begin{array}{r}0,44 \\
(0,06) \\
\end{array}$ \\
\hline
\end{tabular}

Source : calculs de l'auteur.

$\boldsymbol{\Delta}$ : augmentation par rapport à la période précédente (divergence).

$\boldsymbol{\nabla}$ : diminution par rapport à la période précédente (convergence).

HK : Hong Kong, Jap : Japon, Phil : Philippines, Sing : Singapour, Thaï : Thaillande.

Moy : moyenne ; ET : écart-type. 
Tableau 14 : convergence des chocs de demande vers les Philippines

\begin{tabular}{|c|c|c|c|c|c|c|c|c|c|c|c|c|c|c|}
\hline \multirow[b]{2}{*}{$94.1-2003.2$} & \multicolumn{2}{|c|}{ Chine } & \multicolumn{2}{|c|}{ Corée } & \multicolumn{2}{|c|}{ HK } & \multicolumn{2}{|c|}{ Jap } & \multicolumn{2}{|c|}{ Mal } & \multicolumn{2}{|c|}{ Sing } & \multicolumn{2}{|c|}{ Thaï } \\
\hline & $\overline{\alpha \alpha}$ & $\bar{\beta}$ & $\overline{\alpha \alpha}$ & $\bar{\beta}$ & $\bar{\alpha}$ & $\bar{\beta}$ & $\overline{\alpha \alpha}$ & $\bar{\beta}$ & $\overline{\alpha \alpha}$ & $\bar{\beta}$ & $\overline{\alpha \alpha}$ & $\bar{\beta}$ & $\bar{\alpha} \alpha$ & $\bar{\beta}$ \\
\hline $\begin{array}{c}\text { Moy } \\
\text { ET }\end{array}$ & $\begin{array}{c}0,08 \\
(0,34)\end{array}$ & $\begin{array}{c}0,13 \\
(0,91)\end{array}$ & $\begin{array}{c}0,01 \\
(0,58)\end{array}$ & $\begin{array}{c}1,07 \\
(0,43)\end{array}$ & $\begin{array}{l}-0,15 \\
(0,38)\end{array}$ & $\begin{array}{c}1,01 \\
(1,57)\end{array}$ & $\begin{array}{c}0,25 \\
(0,39)\end{array}$ & $\begin{array}{c}1,01 \\
(2,81)\end{array}$ & $\begin{array}{c}0,06 \\
(0,73)\end{array}$ & $\begin{array}{c}0,27 \\
(1,74)\end{array}$ & $\begin{array}{c}0,37 \\
(1,13)\end{array}$ & $\begin{array}{c}-0,80 \\
(4,02)\end{array}$ & $\begin{array}{l}-0,12 \\
(0,74)\end{array}$ & $\begin{array}{c}1,83 \\
(3,11)\end{array}$ \\
\hline $95.1-98.4$ & $\alpha$ & $\beta$ & $\alpha$ & $\beta$ & $\alpha$ & $\beta$ & $\alpha$ & $\beta$ & $\alpha$ & $\beta$ & $\alpha$ & $\beta$ & $\alpha$ & $\beta$ \\
\hline $\begin{array}{c}\text { Moy } \\
\text { ET }\end{array}$ & $\begin{array}{c}0,20 \\
(0,46)\end{array}$ & $\begin{array}{l}-0,09 \\
(1,14)\end{array}$ & $\begin{array}{l}-0,31 \\
(0,62)\end{array}$ & $\begin{array}{c}1,12 \\
(0,38)\end{array}$ & $\begin{array}{l}-0,11 \\
(0,13)\end{array}$ & $\begin{array}{c}0,76 \\
(0,15)\end{array}$ & $\begin{array}{c}0,31 \\
(0,32)\end{array}$ & $\begin{array}{c}0,43 \\
(0,50)\end{array}$ & $\begin{array}{l}-0,26 \\
(0,85)\end{array}$ & $\begin{array}{c}0,50 \\
(0,34)\end{array}$ & $\begin{array}{c}0,78 \\
(1,05)\end{array}$ & $\begin{array}{c}-0,33 \\
(0,97)\end{array}$ & $\begin{array}{l}-0,23 \\
(0,95)\end{array}$ & $\begin{array}{c}1,66 \\
(0,88)\end{array}$ \\
\hline $99.1-2003.2$ & $\alpha$ & $\beta$ & $\alpha$ & $\beta$ & $\alpha$ & $\beta$ & $\alpha$ & $\beta$ & $\alpha$ & $\beta$ & $\alpha$ & $\beta$ & $\alpha$ & $\beta$ \\
\hline $\begin{array}{l}\text { Moy } \\
\text { ET }\end{array}$ & $\begin{array}{c}0,02 \\
(0,11)\end{array}$ & $\begin{array}{r}0,49 \boldsymbol{\Delta} \\
(0,08)\end{array}$ & $\begin{array}{c}0,37 \\
(0,34)\end{array}$ & $\begin{array}{r}0,94 \boldsymbol{\nabla} \\
(0,07)\end{array}$ & $\begin{array}{l}-0,01 \\
(0,03)\end{array}$ & $\begin{array}{r}0,74 \boldsymbol{\nabla} \\
(0,04)\end{array}$ & $\begin{array}{c}0,13 \\
(0,10)\end{array}$ & $\begin{array}{r}0,75 \mathbf{\Delta} \\
(0,09)\end{array}$ & $\begin{array}{c}0,22 \\
(0,43)\end{array}$ & $\begin{array}{r}0,71 \mathbf{\Delta} \\
(0,02)\end{array}$ & $\begin{array}{c}0,41 \\
(0,26)\end{array}$ & $\begin{array}{c}-0,22 \boldsymbol{\nabla} \\
(0,12)\end{array}$ & $\begin{array}{l}-0,03 \\
(0,32)\end{array}$ & $\begin{array}{r}0,94 \boldsymbol{\nabla} \\
(0,11)\end{array}$ \\
\hline
\end{tabular}

Source : calculs de l'auteur.

$\boldsymbol{\Delta}$ : augmentation par rapport à la période précédente (divergence).

$\boldsymbol{\nabla}$ : diminution par rapport à la période précédente (convergence).

HK : Hong Kong, Jap : Japon, Mal : Malaisie, Sing : Singapour, Thaï : Thaïlande.

Moy : moyenne ; ET : écart-type. 
Tableau 15 : convergence des chocs de demande vers Singapour

\begin{tabular}{|c|c|c|c|c|c|c|c|c|c|c|c|c|c|c|}
\hline \multirow[b]{2}{*}{$94.1-2003.2$} & \multicolumn{2}{|c|}{ Chine } & \multicolumn{2}{|c|}{ Corée } & \multicolumn{2}{|c|}{$\mathrm{HK}$} & \multicolumn{2}{|c|}{ Jap } & \multicolumn{2}{|c|}{ Mal } & \multicolumn{2}{|c|}{ Phil } & \multicolumn{2}{|c|}{ Thaï } \\
\hline & $\alpha$ & $\beta$ & $\alpha$ & $\beta$ & $\alpha$ & $\beta$ & $\alpha$ & $\beta$ & $\alpha$ & $\beta$ & $\alpha$ & $\beta$ & $\alpha$ & $\beta$ \\
\hline $\begin{array}{c}\text { Moy } \\
\text { ET }\end{array}$ & $\begin{array}{l}-0,17 \\
(0,64)\end{array}$ & $\begin{array}{c}0,83 \\
(0,19)\end{array}$ & $\begin{array}{c}0,05 \\
(0,55)\end{array}$ & $\begin{array}{c}0,96 \\
(0,24)\end{array}$ & $\begin{array}{l}-0,03 \\
(0,20)\end{array}$ & $\begin{array}{c}0,99 \\
(0,03)\end{array}$ & $\begin{array}{l}-0,06 \\
(0,56)\end{array}$ & $\begin{array}{c}1,07 \\
(0,21)\end{array}$ & $\begin{array}{c}0,03 \\
(0,46)\end{array}$ & $\begin{array}{c}0,99 \\
(0,05)\end{array}$ & $\begin{array}{l}-0,25 \\
(0,30)\end{array}$ & $\begin{array}{c}0,95 \\
(0,07)\end{array}$ & $\begin{array}{l}-0,11 \\
(0,60)\end{array}$ & $\begin{array}{c}0,97 \\
(0,23)\end{array}$ \\
\hline $95.1-98.4$ & $\alpha$ & $\beta$ & $\alpha$ & $\beta$ & $\alpha$ & $\beta$ & $\alpha$ & $\beta$ & $\alpha$ & $\beta$ & $\alpha$ & $\beta$ & $\alpha$ & $\beta$ \\
\hline $\begin{array}{l}\text { Moy } \\
\text { ET }\end{array}$ & $\begin{array}{l}-0,23 \\
(0,49)\end{array}$ & $\begin{array}{c}0,85 \\
(0,10)\end{array}$ & $\begin{array}{l}-0,04 \\
(0,07)\end{array}$ & $\begin{array}{c}0,80 \\
(0,25)\end{array}$ & $\begin{array}{l}-0,05 \\
(0,07)\end{array}$ & $\begin{array}{c}0,98 \\
(0,02)\end{array}$ & $\begin{array}{c}0,06 \\
(0,14)\end{array}$ & $\begin{array}{c}0,99 \\
(0,03)\end{array}$ & $\begin{array}{l}-0,05 \\
(0,16)\end{array}$ & $\begin{array}{c}1,00 \\
(0,01)\end{array}$ & $\begin{array}{l}-0,47 \\
(0,19)\end{array}$ & $\begin{array}{c}0,92 \\
(0,06)\end{array}$ & $\begin{array}{c}0,05 \\
(0,59)\end{array}$ & $\begin{array}{c}0,92 \\
(0,12)\end{array}$ \\
\hline $99.1-2003.2$ & $\alpha$ & $\beta$ & $\alpha$ & $\beta$ & $\alpha$ & $\beta$ & $\alpha$ & $\beta$ & $\alpha$ & $\beta$ & $\alpha$ & $\beta$ & $\alpha$ & $\beta$ \\
\hline $\begin{array}{c}\text { Moy } \\
\text { ET }\end{array}$ & $\begin{array}{c}0,03 \\
(0,07)\end{array}$ & $\begin{array}{r}0,77 \boldsymbol{\nabla} \\
(0,13)\end{array}$ & $\begin{array}{c}0,42 \\
(0,16)\end{array}$ & $\begin{array}{r}1,07 \mathbf{\Delta} \\
(0,18)\end{array}$ & $\begin{array}{c}0,00 \\
(0,05)\end{array}$ & $\begin{array}{r}1,00 \mathbf{\Delta} \\
(0,00)\end{array}$ & $\begin{array}{c}0,12 \\
(0,10)\end{array}$ & $\begin{array}{r}1,02 \mathbf{\Delta} \\
(0,00)\end{array}$ & $\begin{array}{c}-0,09 \\
(0,11)\end{array}$ & $\begin{array}{r}0,98 \boldsymbol{\nabla} \\
(0,00)\end{array}$ & $\begin{array}{c}-0,03 \\
(0,13)\end{array}$ & $\begin{array}{r}0,96 \boldsymbol{\Lambda} \\
(0,00)\end{array}$ & $\begin{array}{c}-0,15 \\
(0,20)\end{array}$ & $\begin{array}{c}1,04 \boldsymbol{\Delta} \\
(0,00)\end{array}$ \\
\hline
\end{tabular}

Source : calculs de l'auteur.

$\boldsymbol{\Delta}$ : augmentation par rapport à la période précédente (divergence).

$\boldsymbol{\nabla}$ : diminution par rapport à la période précédente (convergence).

HK : Hong Kong, Jap : Japon, Mal : Malaisie, Phil : Philippines, Thaï : Thaïlande.

Moy : moyenne ; ET : écart-type. 
Tableau 16 : convergence des chocs de demande vers la Thailande

\begin{tabular}{|c|c|c|c|c|c|c|c|c|c|c|c|c|c|c|}
\hline & \multicolumn{2}{|c|}{ Chine } & \multicolumn{2}{|c|}{ Corée } & \multicolumn{2}{|c|}{ HK } & \multicolumn{2}{|c|}{ Jap } & \multicolumn{2}{|c|}{ Mal } & \multicolumn{2}{|c|}{ Phil } & \multicolumn{2}{|c|}{ Sing } \\
\hline $94.1-2003.2$ & $\alpha$ & $\beta$ & $\alpha$ & $\beta$ & $\alpha$ & $\beta$ & $\alpha$ & $\beta$ & $\alpha$ & $\beta$ & $\alpha$ & $\beta$ & $\alpha$ & $\beta$ \\
\hline $\begin{array}{c}\text { Moy } \\
\text { ET }\end{array}$ & $\begin{array}{c}0,15 \\
(0,54)\end{array}$ & $\begin{array}{c}1,60 \\
(0,45)\end{array}$ & $\begin{array}{c}1,33 \\
(6,85)\end{array}$ & $\begin{array}{c}0,14 \\
(4,94)\end{array}$ & $\begin{array}{l}-1,36 \\
(8,09)\end{array}$ & $\begin{array}{c}1,88 \\
(5,54)\end{array}$ & $\begin{array}{c}0,45 \\
(2,24)\end{array}$ & $\begin{array}{c}0,81 \\
(1,64)\end{array}$ & $\begin{array}{c}1,87 \\
(12,48)\end{array}$ & $\begin{array}{c}-0,51 \\
(8,63)\end{array}$ & $\begin{array}{c}-0,17 \\
(0,29)\end{array}$ & $\begin{array}{c}1,02 \\
(0,15)\end{array}$ & $\begin{array}{c}0,05 \\
(3,34)\end{array}$ & $\begin{array}{c}0,46 \\
(3,14)\end{array}$ \\
\hline $95.1-98.4$ & $\alpha$ & $\beta$ & $\alpha$ & $\beta$ & $\alpha$ & $\beta$ & $\alpha$ & $\beta$ & $\alpha$ & $\beta$ & $\alpha$ & $\beta$ & $\alpha$ & $\beta$ \\
\hline $\begin{array}{l}\text { Moy } \\
\text { ET }\end{array}$ & $\begin{array}{c}0,32 \\
(0,65)\end{array}$ & $\begin{array}{c}1,89 \\
(0,36)\end{array}$ & $\begin{array}{c}-0,09 \\
(0,15)\end{array}$ & $\begin{array}{c}0,79 \\
(0,50)\end{array}$ & $\begin{array}{c}-0,11 \\
(0,08)\end{array}$ & $\begin{array}{c}1,04 \\
(0,06)\end{array}$ & $\begin{array}{c}-0,14 \\
(0,35)\end{array}$ & $\begin{array}{c}1,12 \\
(0,06)\end{array}$ & $\begin{array}{l}-0,17 \\
(0,13)\end{array}$ & $\begin{array}{c}1,01 \\
(0,12)\end{array}$ & $\begin{array}{c}-0,40 \\
(0,25)\end{array}$ & $\begin{array}{c}1,12 \\
(0,13)\end{array}$ & $\begin{array}{c}-0,53 \\
(0,51)\end{array}$ & $\begin{array}{c}1,22 \\
(2,44)\end{array}$ \\
\hline $99.1-2003.2$ & $\alpha$ & $\beta$ & $\alpha$ & $\beta$ & $\alpha$ & $\beta$ & $\alpha$ & $\beta$ & $\alpha$ & $\beta$ & $\alpha$ & $\beta$ & $\alpha$ & $\beta$ \\
\hline $\begin{array}{c}\text { Moy } \\
\text { ET }\end{array}$ & $\begin{array}{c}-0,04 \\
(0,13)\end{array}$ & $\begin{array}{r}1,34 \boldsymbol{\nabla} \\
(0,21)\end{array}$ & $\begin{array}{c}0,60 \\
(0,62)\end{array}$ & $\begin{array}{r}1,16 \mathbf{\Delta} \\
(0,68)\end{array}$ & $\begin{array}{c}0,03 \\
(0,05) \\
\end{array}$ & $\begin{array}{r}0,92 \boldsymbol{\nabla} \\
(0,02)\end{array}$ & $\begin{array}{c}0,29 \\
(0,44)\end{array}$ & $\begin{array}{c}1,14 \boldsymbol{\Lambda} \\
(0,04)\end{array}$ & $\begin{array}{l}-0,15 \\
(0,13)\end{array}$ & $\begin{array}{r}0,85 \boldsymbol{\nabla} \\
(0,01)\end{array}$ & $\begin{array}{c}-0,02 \\
(0,13)\end{array}$ & $\begin{array}{r}0,93 \boldsymbol{\nabla} \\
(0,07)\end{array}$ & $\begin{array}{c}-0,24 \\
(0,57)\end{array}$ & $\begin{array}{r}0,49 \boldsymbol{\nabla} \\
(2,10)\end{array}$ \\
\hline
\end{tabular}

Source : calculs de l'auteur.

$\boldsymbol{\Delta}$ : augmentation par rapport à la période précédente (divergence)

$\boldsymbol{\nabla}$ : diminution par rapport à la période précédente (convergence)

HK : Hong Kong, Jap : Japon, Mal : Malaisie, Phil : Philippines, Sing : Singapour

Moy : moyenne ; ET : écart-type. 
Tableau 17 : convergence des chocs d'offre vers la Chine

\begin{tabular}{|c|c|c|c|c|c|c|c|c|c|c|c|c|c|c|}
\hline \multirow[b]{2}{*}{$94.1-2003.2$} & \multicolumn{2}{|c|}{ Corée } & \multicolumn{2}{|c|}{ HK } & \multicolumn{2}{|c|}{ Jap } & \multicolumn{2}{|c|}{ Mal } & \multicolumn{2}{|c|}{ Phil } & \multicolumn{2}{|c|}{ Sing } & \multicolumn{2}{|c|}{ Thaï } \\
\hline & $\alpha$ & $\beta$ & $\alpha$ & $\beta$ & $\alpha$ & $\beta$ & $\alpha$ & $\beta$ & $\alpha$ & $\beta$ & $\alpha$ & $\beta$ & $\alpha$ & $\beta$ \\
\hline $\begin{array}{c}\text { Moy } \\
\text { ET }\end{array}$ & $\begin{array}{l}-0,12 \\
(0,24)\end{array}$ & $\begin{array}{c}0,33 \\
(0,48)\end{array}$ & $\begin{array}{l}-0,01 \\
(0,11)\end{array}$ & $\begin{array}{c}0,19 \\
(0,43)\end{array}$ & $\begin{array}{c}0,03 \\
(0,19)\end{array}$ & $\begin{array}{c}0,39 \\
(0,49)\end{array}$ & $\begin{array}{l}-0,22 \\
(0,44)\end{array}$ & $\begin{array}{c}0,02 \\
(1,41)\end{array}$ & $\begin{array}{c}0,06 \\
(0,30)\end{array}$ & $\begin{array}{c}0,38 \\
(0,16)\end{array}$ & $\begin{array}{c}0,04 \\
(0,16)\end{array}$ & $\begin{array}{c}-0,09 \\
(0,70)\end{array}$ & $\begin{array}{l}-0,01 \\
(0,10)\end{array}$ & $\begin{array}{c}0,13 \\
(0,26\end{array}$ \\
\hline $95.1-98.4$ & $\alpha$ & $\beta$ & $\alpha$ & $\beta$ & $\alpha$ & $\beta$ & $\alpha$ & $\beta$ & $\alpha$ & $\beta$ & $\alpha$ & $\beta$ & $\alpha$ & $\beta$ \\
\hline $\begin{array}{c}\text { Moy } \\
\text { ET }\end{array}$ & $\begin{array}{l}-0,23 \\
(0,19)\end{array}$ & $\begin{array}{c}0,30 \\
(0,50)\end{array}$ & $\begin{array}{c}0,01 \\
(0,14)\end{array}$ & $\begin{array}{c}0,12 \\
(0,46)\end{array}$ & $\begin{array}{c}0,08 \\
(0,23)\end{array}$ & $\begin{array}{c}0,08 \\
(0,46)\end{array}$ & $\begin{array}{l}-0,27 \\
(0,24)\end{array}$ & $\begin{array}{c}0,04 \\
(1,60)\end{array}$ & $\begin{array}{c}0,03 \\
(0,25)\end{array}$ & $\begin{array}{c}0,41 \\
(0,16)\end{array}$ & $\begin{array}{c}0,07 \\
(0,11)\end{array}$ & $\begin{array}{l}-0,22 \\
(0,71)\end{array}$ & $\begin{array}{l}-0,04 \\
(0,07)\end{array}$ & $\begin{array}{c}0,01 \\
(0,22)\end{array}$ \\
\hline $99.1-2003.2$ & $\alpha$ & $\beta$ & $\alpha$ & $\beta$ & $\alpha$ & $\beta$ & $\alpha$ & $\beta$ & $\alpha$ & $\beta$ & $\alpha$ & $\beta$ & $\alpha$ & $\beta$ \\
\hline $\begin{array}{c}\text { Moy } \\
\text { ET }\end{array}$ & $\begin{array}{c}0,06 \\
(0,04) \\
\end{array}$ & $\begin{array}{r}0,44 \boldsymbol{\Lambda} \\
(0,40) \\
\end{array}$ & $\begin{array}{c}-0,01 \\
(0,05) \\
\end{array}$ & $\begin{array}{r}0,35 \mathbf{\Delta} \\
(0,03) \\
\end{array}$ & $\begin{array}{c}-0,06 \\
(0,04) \\
\end{array}$ & $\begin{array}{r}0,75 \mathbf{\Delta} \\
(0,13) \\
\end{array}$ & $\begin{array}{l}-0,01 \\
(0,05) \\
\end{array}$ & $\begin{array}{r}0,31 \boldsymbol{\Delta} \\
(0,33) \\
\end{array}$ & $\begin{array}{c}0,14 \\
(0,32) \\
\end{array}$ & $\begin{array}{c}0,41 \\
(0,05) \\
\end{array}$ & $\begin{array}{c}0,06 \\
(0,02) \\
\end{array}$ & $\begin{array}{r}0,18 \boldsymbol{\Delta} \\
(0,21) \\
\end{array}$ & $\begin{array}{c}0,04 \\
(0,03) \\
\end{array}$ & $\begin{array}{r}0,29 \boldsymbol{\Delta} \\
(0,05) \\
\end{array}$ \\
\hline
\end{tabular}

Source : calculs de l'auteur.

$\boldsymbol{\Delta}$ : augmentation par rapport à la période précédente (divergence).

$\boldsymbol{\nabla}$ : diminution par rapport à la période précédente (convergence).

HK : Hong Kong, Jap : Japon, Mal : Malaisie, Phil : Philippines, Sing : Singapour, Thaï : Thaïlande.

Moy : moyenne; ET : écart-type. 
Tableau 18 : convergence des chocs d'offre vers la Corée du Sud

\begin{tabular}{|c|c|c|c|c|c|c|c|c|c|c|c|c|c|c|}
\hline \multirow[b]{2}{*}{$94.1-2003.2$} & \multicolumn{2}{|c|}{ Chine } & \multicolumn{2}{|c|}{ HK } & \multicolumn{2}{|c|}{ Jap } & \multicolumn{2}{|c|}{ Mal } & \multicolumn{2}{|c|}{ Phil } & \multicolumn{2}{|c|}{ Sing } & \multicolumn{2}{|c|}{ Thaï } \\
\hline & $\alpha$ & $\beta$ & $\alpha$ & $\beta$ & $\alpha$ & $\beta$ & $\alpha$ & $\beta$ & $\alpha$ & $\beta$ & $\alpha$ & $\beta$ & $\alpha$ & $\beta$ \\
\hline $\begin{array}{c}\text { Moy } \\
\text { ET }\end{array}$ & $\begin{array}{c}0,12 \\
(0,17)\end{array}$ & $\begin{array}{c}0,11 \\
(0,12)\end{array}$ & $\begin{array}{c}0,12 \\
(0,19)\end{array}$ & $\begin{array}{c}0,15 \\
(0,17)\end{array}$ & $\begin{array}{c}0,23 \\
(0,42)\end{array}$ & $\begin{array}{c}0,39 \\
(0,61)\end{array}$ & $\begin{array}{c}0,16 \\
(0,24)\end{array}$ & $\begin{array}{l}-0,11 \\
(0,22)\end{array}$ & $\begin{array}{c}0,09 \\
(0,11)\end{array}$ & $\begin{array}{c}0,34 \\
(0,12)\end{array}$ & $\begin{array}{l}-0,03 \\
(1,02)\end{array}$ & $\begin{array}{l}-0,19 \\
(2,58)\end{array}$ & $\begin{array}{c}0,05 \\
(0,41)\end{array}$ & $\begin{array}{c}0,21 \\
(0,25)\end{array}$ \\
\hline $95.1-98.4$ & $\alpha$ & $\beta$ & $\alpha$ & $\beta$ & $\alpha$ & $\beta$ & $\alpha$ & $\beta$ & $\alpha$ & $\beta$ & $\alpha$ & $\beta$ & $\alpha$ & $\beta$ \\
\hline $\begin{array}{l}\text { Moy } \\
\text { ET }\end{array}$ & $\begin{array}{c}0,24 \\
(0,13)\end{array}$ & $\begin{array}{c}0,04 \\
(0,12)\end{array}$ & $\begin{array}{c}0,23 \\
(0,09)\end{array}$ & $\begin{array}{c}0,06 \\
(0,06)\end{array}$ & $\begin{array}{c}0,32 \\
(0,31)\end{array}$ & $\begin{array}{c}0,15 \\
(0,49)\end{array}$ & $\begin{array}{c}0,22 \\
(0,20)\end{array}$ & $\begin{array}{l}-0,25 \\
(0,17)\end{array}$ & $\begin{array}{c}0,09 \\
(0,10)\end{array}$ & $\begin{array}{c}0,42 \\
(0,08)\end{array}$ & $\begin{array}{c}0,25 \\
(0,10)\end{array}$ & $\begin{array}{c}0,07 \\
(0,12)\end{array}$ & $\begin{array}{c}0,11 \\
(0,40)\end{array}$ & $\begin{array}{l}-0,01 \\
(0,11)\end{array}$ \\
\hline $99.1-2003.2$ & $\alpha$ & $\beta$ & $\alpha$ & $\beta$ & $\alpha$ & $\beta$ & $\alpha$ & $\beta$ & $\alpha$ & $\beta$ & $\alpha$ & $\beta$ & $\alpha$ & $\beta$ \\
\hline $\begin{array}{c}\text { Moy } \\
\text { ET } \\
\end{array}$ & $\begin{array}{l}-0,02 \\
(0,04) \\
\end{array}$ & $\begin{array}{r}0,14 \boldsymbol{\Lambda} \\
(0,03) \\
\end{array}$ & $\begin{array}{l}-0,01 \\
(0,05) \\
\end{array}$ & $\begin{array}{r}0,27 \mathbf{\Delta} \\
(0,16) \\
\end{array}$ & $\begin{array}{l}-0,06 \\
(0,10) \\
\end{array}$ & $\begin{array}{r}0,85 \boldsymbol{\Delta} \\
(0,13) \\
\end{array}$ & $\begin{array}{c}0,02 \\
(0,03) \\
\end{array}$ & $\begin{array}{r}0,00 \boldsymbol{\Delta} \\
(0,17) \\
\end{array}$ & $\begin{array}{c}0,12 \\
(0,11) \\
\end{array}$ & $\begin{array}{r}0,26 \nabla \\
(0,03) \\
\end{array}$ & $\begin{array}{c}0,08 \\
(0,03) \\
\end{array}$ & $\begin{array}{r}0,26 \boldsymbol{\Delta} \\
(0,08) \\
\end{array}$ & $\begin{array}{c}-0,14 \\
(0,29) \\
\end{array}$ & $\begin{array}{r}0,44 \mathbf{\Delta} \\
(0,11) \\
\end{array}$ \\
\hline
\end{tabular}

Source : calculs de l'auteur.

$\boldsymbol{\Delta}$ : augmentation par rapport à la période précédente (divergence).

$\boldsymbol{\nabla}$ : diminution par rapport à la période précédente (convergence).

HK : Hong Kong, Jap : Japon, Mal : Malaisie, Phil : Philippines, Sing : Singapour, Thaï : Thaïlande.

Moy : moyenne ; ET : écart-type. 
Tableau 19: convergence des chocs d'offre vers Hong Kong

\begin{tabular}{|c|c|c|c|c|c|c|c|c|c|c|c|c|c|c|}
\hline \multirow[b]{2}{*}{$94.1-2003.2$} & \multicolumn{2}{|c|}{ Chine } & \multicolumn{2}{|c|}{ Corée } & \multicolumn{2}{|c|}{ Jap } & \multicolumn{2}{|c|}{ Mal } & \multicolumn{2}{|c|}{ Phil } & \multicolumn{2}{|c|}{ Sing } & \multicolumn{2}{|c|}{ Thaï } \\
\hline & $\alpha$ & $\beta$ & $\alpha$ & $\beta$ & $\alpha$ & $\beta$ & $\alpha$ & $\beta$ & $\alpha$ & $\beta$ & $\alpha$ & $\beta$ & $\alpha$ & $\beta$ \\
\hline $\begin{array}{c}\text { Moy } \\
\text { ET }\end{array}$ & $\begin{array}{c}0,03 \\
(0,09)\end{array}$ & $\begin{array}{c}0,31 \\
(0,14)\end{array}$ & $\begin{array}{l}-0,10 \\
(0,29)\end{array}$ & $\begin{array}{c}0,36 \\
(0,32)\end{array}$ & $\begin{array}{l}-0,04 \\
(0,12)\end{array}$ & $\begin{array}{c}0,45 \\
(0,42)\end{array}$ & $\begin{array}{c}0,01 \\
(0,16)\end{array}$ & $\begin{array}{c}0,08 \\
(0,19)\end{array}$ & $\begin{array}{c}0,05 \\
(0,28)\end{array}$ & $\begin{array}{c}0,62 \\
(0,13)\end{array}$ & $\begin{array}{c}0,00 \\
(0,16)\end{array}$ & $\begin{array}{c}0,33 \\
(0,19)\end{array}$ & $\begin{array}{c}0,05 \\
(0,48)\end{array}$ & $\begin{array}{c}0,35 \\
(0,14)\end{array}$ \\
\hline $95.1-98.4$ & $\alpha$ & $\beta$ & $\alpha$ & $\beta$ & $\alpha$ & $\beta$ & $\alpha$ & $\beta$ & $\alpha$ & $\beta$ & $\alpha$ & $\beta$ & $\alpha$ & $\beta$ \\
\hline $\begin{array}{l}\text { Moy } \\
\text { ET }\end{array}$ & $\begin{array}{c}0,02 \\
(0,12)\end{array}$ & $\begin{array}{c}0,23 \\
(0,15)\end{array}$ & $\begin{array}{l}-0,22 \\
(0,12)\end{array}$ & $\begin{array}{c}0,27 \\
(0,08)\end{array}$ & $\begin{array}{l}-0,03 \\
(0,14)\end{array}$ & $\begin{array}{c}0,16 \\
(0,33)\end{array}$ & $\begin{array}{l}-0,09 \\
(0,07)\end{array}$ & $\begin{array}{l}-0,04 \\
(0,12)\end{array}$ & $\begin{array}{c}0,02 \\
(0,21)\end{array}$ & $\begin{array}{c}0,60 \\
(0,14)\end{array}$ & $\begin{array}{c}0,02 \\
(0,12)\end{array}$ & $\begin{array}{c}0,23 \\
(0,12)\end{array}$ & $\begin{array}{c}0,04 \\
(0,46)\end{array}$ & $\begin{array}{c}0,25 \\
(0,13)\end{array}$ \\
\hline $99.1-2003.2$ & $\alpha$ & $\beta$ & $\alpha$ & $\beta$ & $\alpha$ & $\beta$ & $\alpha$ & $\beta$ & $\alpha$ & $\beta$ & $\alpha$ & $\beta$ & $\alpha$ & $\beta$ \\
\hline $\begin{array}{c}\text { Moy } \\
\text { ET }\end{array}$ & $\begin{array}{c}0,04 \\
(0,05)\end{array}$ & $\begin{array}{r}0,38 \boldsymbol{\Delta} \\
(0,05)\end{array}$ & $\begin{array}{c}0,00 \\
(0,03)\end{array}$ & $\begin{array}{r}0,53 \mathbf{\Delta} \\
(0,17)\end{array}$ & $\begin{array}{c}-0,08 \\
(0,04)\end{array}$ & $\begin{array}{r}0,83 \boldsymbol{\Delta} \\
(0,11)\end{array}$ & $\begin{array}{c}0,02 \\
(0,04)\end{array}$ & $\begin{array}{r}0,25 \mathbf{\Delta} \\
(0,13)\end{array}$ & $\begin{array}{c}0,15 \\
(0,30)\end{array}$ & $\begin{array}{r}0,67 \boldsymbol{\Delta} \\
(0,05)\end{array}$ & $\begin{array}{c}0,07 \\
(0,03)\end{array}$ & $\begin{array}{r}0,47 \boldsymbol{\Lambda} \\
(0,09)\end{array}$ & $\begin{array}{c}-0,03 \\
(0,51)\end{array}$ & $\begin{array}{r}0,45 \boldsymbol{\Delta} \\
(0,03)\end{array}$ \\
\hline
\end{tabular}

Source : calculs de l'auteur.

$\boldsymbol{\Delta}$ : augmentation par rapport à la période précédente (divergence).

$\boldsymbol{\nabla}$ : diminution par rapport à la période précédente (convergence).

Jap : Japon, Mal : Malaisie, Phil : Philippines, Sing : Singapour, Thaï : Thaïlande.

Moy : moyenne ; ET : écart-type. 
Tableau 20 : convergence des chocs d'offre vers le Japon

\begin{tabular}{|c|c|c|c|c|c|c|c|c|c|c|c|c|c|c|}
\hline \multirow[b]{2}{*}{$94.1-2003.2$} & \multicolumn{2}{|c|}{ Chine } & \multicolumn{2}{|c|}{ Corée } & \multicolumn{2}{|c|}{$\mathrm{HK}$} & \multicolumn{2}{|c|}{ Mal } & \multicolumn{2}{|c|}{ Phil } & \multicolumn{2}{|c|}{ Sing } & \multicolumn{2}{|c|}{ Thaï } \\
\hline & $\alpha$ & $\beta$ & $\alpha$ & $\beta$ & $\alpha$ & $\beta$ & $\alpha$ & $\beta$ & $\alpha$ & $\beta$ & $\alpha$ & $\beta$ & $\alpha$ & $\beta$ \\
\hline $\begin{array}{c}\text { Moy } \\
\text { ET }\end{array}$ & $\begin{array}{c}0,04 \\
(0,18)\end{array}$ & $\begin{array}{c}0,43 \\
(0,07)\end{array}$ & $\begin{array}{l}-0,08 \\
(0,21)\end{array}$ & $\begin{array}{c}0,61 \\
(0,06)\end{array}$ & $\begin{array}{c}0,08 \\
(0,12)\end{array}$ & $\begin{array}{c}0,42 \\
(0,13)\end{array}$ & $\begin{array}{c}0,01 \\
(0,20)\end{array}$ & $\begin{array}{c}0,29 \\
(0,19)\end{array}$ & $\begin{array}{c}0,10 \\
(0,28)\end{array}$ & $\begin{array}{c}0,83 \\
(0,22)\end{array}$ & $\begin{array}{c}0,14 \\
(0,24)\end{array}$ & $\begin{array}{c}0,53 \\
(0,13)\end{array}$ & $\begin{array}{c}0,14 \\
(0,25)\end{array}$ & $\begin{array}{c}0,85 \\
(1,04)\end{array}$ \\
\hline $95.1-98.4$ & $\alpha$ & $\beta$ & $\alpha$ & $\beta$ & $\alpha$ & $\beta$ & $\alpha$ & $\beta$ & $\alpha$ & $\beta$ & $\alpha$ & $\beta$ & $\alpha$ & $\beta$ \\
\hline $\begin{array}{l}\text { Moy } \\
\text { ET }\end{array}$ & $\begin{array}{c}0,05 \\
(0,23)\end{array}$ & $\begin{array}{c}0,44 \\
(0,04)\end{array}$ & $\begin{array}{l}-0,17 \\
(0,23)\end{array}$ & $\begin{array}{c}0,56 \\
(0,06)\end{array}$ & $\begin{array}{c}0,13 \\
(0,13)\end{array}$ & $\begin{array}{c}0,43 \\
(0,07)\end{array}$ & $\begin{array}{l}-0,09 \\
(0,16)\end{array}$ & $\begin{array}{c}0,17 \\
(0,16)\end{array}$ & $\begin{array}{c}0,08 \\
(0,24)\end{array}$ & $\begin{array}{c}0,78 \\
(0,06)\end{array}$ & $\begin{array}{c}0,26 \\
(0,30)\end{array}$ & $\begin{array}{c}0,51 \\
(0,07)\end{array}$ & $\begin{array}{c}0,20 \\
(0,15)\end{array}$ & $\begin{array}{c}0,65 \\
(0,21)\end{array}$ \\
\hline $99.1-2003.2$ & $\alpha$ & $\beta$ & $\alpha$ & $\beta$ & $\alpha$ & $\beta$ & $\alpha$ & $\beta$ & $\alpha$ & $\beta$ & $\alpha$ & $\beta$ & $\alpha$ & $\beta$ \\
\hline $\begin{array}{c}\text { Moy } \\
\text { ET } \\
\end{array}$ & $\begin{array}{c}0,07 \\
(0,05) \\
\end{array}$ & $\begin{array}{r}0,45 \boldsymbol{\Delta} \\
(0,02) \\
\end{array}$ & $\begin{array}{c}0,06 \\
(0,03) \\
\end{array}$ & $\begin{array}{r}0,63 \mathbf{\Delta} \\
(0,02) \\
\end{array}$ & $\begin{array}{c}0,07 \\
(0,05) \\
\end{array}$ & $\begin{array}{r}0,49 \boldsymbol{\Delta} \\
(0,02) \\
\end{array}$ & $\begin{array}{c}0,06 \\
(0,03) \\
\end{array}$ & $\begin{array}{r}0,45 \boldsymbol{\Delta} \\
(0,04) \\
\end{array}$ & $\begin{array}{c}0,14 \\
(0,30) \\
\end{array}$ & $\begin{array}{r}0,85 \boldsymbol{\Delta} \\
(0,02) \\
\end{array}$ & $\begin{array}{c}0,05 \\
(0,10) \\
\end{array}$ & $\begin{array}{r}0,47 \boldsymbol{\nabla} \\
(0,01) \\
\end{array}$ & $\begin{array}{c}0,15 \\
(0,06) \\
\end{array}$ & $\begin{array}{r}0,61 \nabla \\
(0,13) \\
\end{array}$ \\
\hline
\end{tabular}

Source : calculs de l'auteur.

$\boldsymbol{\Delta}$ : augmentation par rapport à la période précédente (divergence).

$\boldsymbol{\nabla}$ : diminution par rapport à la période précédente (convergence).

HK : Hong Kong, Mal : Malaisie, Phil : Philippines, Sing : Singapour, Thaï : Thaïlande.

Moy : moyenne ; ET : écart-type. 
Tableau 21 : convergence des chocs d'offre vers la Malaisie

\begin{tabular}{|c|c|c|c|c|c|c|c|c|c|c|c|c|c|c|}
\hline \multirow[b]{2}{*}{$94.1-2003.2$} & \multicolumn{2}{|c|}{ Chine } & \multicolumn{2}{|c|}{ Corée } & \multicolumn{2}{|c|}{$\mathrm{HK}$} & \multicolumn{2}{|c|}{ Jap } & \multicolumn{2}{|c|}{ Phil } & \multicolumn{2}{|c|}{ Sing } & \multicolumn{2}{|c|}{ Thaï } \\
\hline & $\alpha$ & $\beta$ & $\alpha$ & $\beta$ & $\alpha$ & $\beta$ & $\alpha$ & $\beta$ & $\alpha$ & $\beta$ & $\alpha$ & $\beta$ & $\alpha$ & $\beta$ \\
\hline $\begin{array}{c}\text { Moy } \\
\text { ET }\end{array}$ & $\begin{array}{c}0,15 \\
(0,14)\end{array}$ & $\begin{array}{c}0,38 \\
(0,44)\end{array}$ & $\begin{array}{l}-0,11 \\
(0,19)\end{array}$ & $\begin{array}{c}0,40 \\
(0,20)\end{array}$ & $\begin{array}{c}0,00 \\
(0,14)\end{array}$ & $\begin{array}{c}0,36 \\
(0,11)\end{array}$ & $\begin{array}{c}0,04 \\
(0,24)\end{array}$ & $\begin{array}{c}0,54 \\
(0,46)\end{array}$ & $\begin{array}{c}0,12 \\
(0,23)\end{array}$ & $\begin{array}{c}0,62 \\
(0,13)\end{array}$ & $\begin{array}{c}0,02 \\
(0,24)\end{array}$ & $\begin{array}{c}0,46 \\
(0,33)\end{array}$ & $\begin{array}{c}0,16 \\
(0,22)\end{array}$ & $\begin{array}{c}0,55 \\
(0,32)\end{array}$ \\
\hline $95.1-98.4$ & $\alpha$ & $\beta$ & $\alpha$ & $\beta$ & $\alpha$ & $\beta$ & $\alpha$ & $\beta$ & $\alpha$ & $\beta$ & $\alpha$ & $\beta$ & $\alpha$ & $\beta$ \\
\hline $\begin{array}{l}\text { Moy } \\
\text { ET }\end{array}$ & $\begin{array}{c}0,23 \\
(0,12)\end{array}$ & $\begin{array}{c}0,49 \\
(0,56)\end{array}$ & $\begin{array}{l}-0,16 \\
(0,15)\end{array}$ & $\begin{array}{c}0,47 \\
(0,06)\end{array}$ & $\begin{array}{c}0,08 \\
(0,07)\end{array}$ & $\begin{array}{c}0,42 \\
(0,06)\end{array}$ & $\begin{array}{c}0,16 \\
(0,11)\end{array}$ & $\begin{array}{c}0,37 \\
(0,49)\end{array}$ & $\begin{array}{c}0,13 \\
(0,17)\end{array}$ & $\begin{array}{c}0,67 \\
(0,05)\end{array}$ & $\begin{array}{c}0,12 \\
(0,16)\end{array}$ & $\begin{array}{c}0,46 \\
(0,43)\end{array}$ & $\begin{array}{c}0,26 \\
(0,13)\end{array}$ & $\begin{array}{c}0,49 \\
(0,26)\end{array}$ \\
\hline $99.1-2003.2$ & $\alpha$ & $\beta$ & $\alpha$ & $\beta$ & $\alpha$ & $\beta$ & $\alpha$ & $\beta$ & $\alpha$ & $\beta$ & $\alpha$ & $\beta$ & $\alpha$ & $\beta$ \\
\hline $\begin{array}{c}\text { Moy } \\
\text { ET }\end{array}$ & $\begin{array}{c}0,07 \\
(0,10)\end{array}$ & $\begin{array}{c}0,23 \boldsymbol{\nabla} \\
(0,26)\end{array}$ & $\begin{array}{c}0,00 \\
(0,02)\end{array}$ & $\begin{array}{c}0,39 \boldsymbol{\nabla} \\
(0,03)\end{array}$ & $\begin{array}{c}-0,01 \\
(0,05)\end{array}$ & $\begin{array}{c}0,36 \boldsymbol{\nabla} \\
(0,03)\end{array}$ & $\begin{array}{c}0,00 \\
(0,06)\end{array}$ & $\begin{array}{r}0,80 \mathbf{\Delta} \\
(0,22)\end{array}$ & $\begin{array}{c}0,12 \\
(0,29)\end{array}$ & $\begin{array}{c}0,58 \boldsymbol{\nabla} \\
(0,03)\end{array}$ & $\begin{array}{c}0,10 \\
(0,05)\end{array}$ & $\begin{array}{r}0,50 \boldsymbol{\Delta} \\
(0,22)\end{array}$ & $\begin{array}{c}0,02 \\
(0,14)\end{array}$ & $\begin{array}{r}0,54 \boldsymbol{\Delta} \\
(0,23)\end{array}$ \\
\hline
\end{tabular}

Source : calculs de l'auteur.

$\boldsymbol{\Delta}$ : augmentation par rapport à la période précédente (divergence).

$\boldsymbol{\nabla}$ : diminution par rapport à la période précédente (convergence).

HK : Hong Kong, Jap : Japon, Phil : Philippines, Sing : Singapour, Thaï : Thaïlande.

Moy : moyenne ; ET : écart-type. 
Tableau 22 : convergence des chocs d'offre vers les Philippines

\begin{tabular}{|c|c|c|c|c|c|c|c|c|c|c|c|c|c|c|}
\hline \multirow[b]{2}{*}{$94.1-2003.2$} & \multicolumn{2}{|c|}{ Chine } & \multicolumn{2}{|c|}{ Corée } & \multicolumn{2}{|c|}{ HK } & \multicolumn{2}{|c|}{ Jap } & \multicolumn{2}{|c|}{ Mal } & \multicolumn{2}{|c|}{ Sing } & \multicolumn{2}{|c|}{ Thaï } \\
\hline & $\alpha$ & $\beta$ & $\alpha$ & $\beta$ & $\alpha$ & $\beta$ & $\alpha$ & $\beta$ & $\alpha$ & $\beta$ & $\alpha$ & $\beta$ & $\alpha$ & $\beta$ \\
\hline $\begin{array}{c}\text { Moy } \\
\text { ET }\end{array}$ & $\begin{array}{c}0,08 \\
(0,11)\end{array}$ & $\begin{array}{c}0,30 \\
(0,42)\end{array}$ & $\begin{array}{l}-0,03 \\
(0,13)\end{array}$ & $\begin{array}{c}0,23 \\
(0,45)\end{array}$ & $\begin{array}{c}0,10 \\
(0,17)\end{array}$ & $\begin{array}{c}0,40 \\
(0,42)\end{array}$ & $\begin{array}{c}-0,02 \\
(0,18)\end{array}$ & $\begin{array}{c}0,51 \\
(0,65)\end{array}$ & $\begin{array}{l}-0,15 \\
(0,12)\end{array}$ & $\begin{array}{c}-0,20 \\
(1,21)\end{array}$ & $\begin{array}{c}0,23 \\
(0,23)\end{array}$ & $\begin{array}{c}0,11 \\
(0,79)\end{array}$ & $\begin{array}{c}0,19 \\
(0,22)\end{array}$ & $\begin{array}{c}0,30 \\
(0,65)\end{array}$ \\
\hline $95.1-98.4$ & $\alpha$ & $\beta$ & $\alpha$ & $\beta$ & $\alpha$ & $\beta$ & $\alpha$ & $\beta$ & $\alpha$ & $\beta$ & $\alpha$ & $\beta$ & $\alpha$ & $\beta$ \\
\hline $\begin{array}{l}\text { Moy } \\
\text { ET }\end{array}$ & $\begin{array}{c}0,12 \\
(0,11)\end{array}$ & $\begin{array}{c}0,14 \\
(0,53)\end{array}$ & $\begin{array}{c}-0,10 \\
(0,15)\end{array}$ & $\begin{array}{c}0,11 \\
(0,34)\end{array}$ & $\begin{array}{c}0,14 \\
(0,08)\end{array}$ & $\begin{array}{c}0,30 \\
(0,22)\end{array}$ & $\begin{array}{c}0,05 \\
(0,18)\end{array}$ & $\begin{array}{c}0,14 \\
(0,70)\end{array}$ & $\begin{array}{c}-0,20 \\
(0,11)\end{array}$ & $\begin{array}{c}-0,49 \\
(0,92)\end{array}$ & $\begin{array}{c}0,26 \\
(0,14)\end{array}$ & $\begin{array}{l}-0,07 \\
(0,47)\end{array}$ & $\begin{array}{c}0,17 \\
(0,11)\end{array}$ & $\begin{array}{c}0,10 \\
(0,51)\end{array}$ \\
\hline $99.1-2003.2$ & $\alpha$ & $\beta$ & $\alpha$ & $\beta$ & $\alpha$ & $\beta$ & $\alpha$ & $\beta$ & $\alpha$ & $\beta$ & $\alpha$ & $\beta$ & $\alpha$ & $\beta$ \\
\hline $\begin{array}{c}\text { Moy } \\
\text { ET }\end{array}$ & $\begin{array}{c}0,02 \\
(0,05)\end{array}$ & $\begin{array}{c}0,47 \boldsymbol{\Lambda} \\
(0,23)\end{array}$ & $\begin{array}{c}0,01 \\
(0,03)\end{array}$ & $\begin{array}{c}0,50 \boldsymbol{\Lambda} \\
(0,22)\end{array}$ & $\begin{array}{c}-0,02 \\
(0,05)\end{array}$ & $\begin{array}{c}0,67 \boldsymbol{\Lambda} \\
(0,05)\end{array}$ & $\begin{array}{c}-0,07 \\
(0,04)\end{array}$ & $\begin{array}{c}0,84 \boldsymbol{\Lambda} \\
(0,03)\end{array}$ & $\begin{array}{l}-0,13 \\
(0,07) \\
\end{array}$ & $\begin{array}{r}0,50 \boldsymbol{\Lambda} \\
(0,10)\end{array}$ & $\begin{array}{c}0,09 \\
(0,04) \\
\end{array}$ & $\begin{array}{r}0,60 \boldsymbol{\Delta} \\
(0,05)\end{array}$ & $\begin{array}{c}0,10 \\
(0,04)\end{array}$ & $\begin{array}{c}0,72 \wedge \\
(0,14)\end{array}$ \\
\hline
\end{tabular}

Source : calculs de l'auteur.

$\boldsymbol{\Delta}$ : augmentation par rapport à la période précédente (divergence).

$\boldsymbol{\nabla}$ : diminution par rapport à la période précédente (convergence).

HK : Hong Kong, Jap : Japon, Mal : Malaisie, Sing : Singapour, Thaï : Thaïlande.

Moy : moyenne ; ET : écart-type. 
Tableau 23 : convergence des chocs d'offre vers Singapour

\begin{tabular}{|c|c|c|c|c|c|c|c|c|c|c|c|c|c|c|}
\hline \multirow[b]{2}{*}{$94.1-2003.2$} & \multicolumn{2}{|c|}{ Chine } & \multicolumn{2}{|c|}{ Corée } & \multicolumn{2}{|c|}{$\mathrm{HK}$} & \multicolumn{2}{|c|}{ Jap } & \multicolumn{2}{|c|}{ Mal } & \multicolumn{2}{|c|}{ Phil } & \multicolumn{2}{|c|}{ Thaï } \\
\hline & $\alpha$ & $\beta$ & $\alpha$ & $\beta$ & $\alpha$ & $\beta$ & $\alpha$ & $\beta$ & $\alpha$ & $\beta$ & $\alpha$ & $\beta$ & $\alpha$ & $\beta$ \\
\hline $\begin{array}{c}\text { Moy } \\
\text { ET }\end{array}$ & $\begin{array}{c}0,03 \\
(0,10)\end{array}$ & $\begin{array}{c}0,22 \\
(0,28)\end{array}$ & $\begin{array}{l}-0,05 \\
(0,26)\end{array}$ & $\begin{array}{c}0,36 \\
(0,39)\end{array}$ & $\begin{array}{c}0,07 \\
(0,17)\end{array}$ & $\begin{array}{c}0,37 \\
(0,31)\end{array}$ & $\begin{array}{c}0,08 \\
(0,29)\end{array}$ & $\begin{array}{c}0,38 \\
(0,88)\end{array}$ & $\begin{array}{c}0,07 \\
(0,29)\end{array}$ & $\begin{array}{c}0,21 \\
(0,65)\end{array}$ & $\begin{array}{c}0,02 \\
(0,30)\end{array}$ & $\begin{array}{c}0,57 \\
(0,23)\end{array}$ & $\begin{array}{c}0,09 \\
(0,09)\end{array}$ & $\begin{array}{c}0,36 \\
(0,30)\end{array}$ \\
\hline $95.1-98.4$ & $\alpha$ & $\beta$ & $\alpha$ & $\beta$ & $\alpha$ & $\beta$ & $\alpha$ & $\beta$ & $\alpha$ & $\beta$ & $\alpha$ & $\beta$ & $\alpha$ & $\beta$ \\
\hline $\begin{array}{l}\text { Moy } \\
\text { ET }\end{array}$ & $\begin{array}{c}0,01 \\
(0,11)\end{array}$ & $\begin{array}{c}0,32 \\
(0,30)\end{array}$ & $\begin{array}{l}-0,19 \\
(0,11)\end{array}$ & $\begin{array}{c}0,45 \\
(0,44)\end{array}$ & $\begin{array}{c}0,06 \\
(0,07)\end{array}$ & $\begin{array}{c}0,39 \\
(0,30)\end{array}$ & $\begin{array}{c}0,05 \\
(0,16)\end{array}$ & $\begin{array}{c}0,31 \\
(0,52)\end{array}$ & $\begin{array}{l}-0,03 \\
(0,07)\end{array}$ & $\begin{array}{c}0,36 \\
(0,87)\end{array}$ & $\begin{array}{c}0,00 \\
(0,25)\end{array}$ & $\begin{array}{c}0,65 \\
(0,21)\end{array}$ & $\begin{array}{c}0,06 \\
(0,09)\end{array}$ & $\begin{array}{c}0,35 \\
(0,37)\end{array}$ \\
\hline $99.1-2003.2$ & $\alpha$ & $\beta$ & $\alpha$ & $\beta$ & $\alpha$ & $\beta$ & $\alpha$ & $\beta$ & $\alpha$ & $\beta$ & $\alpha$ & $\beta$ & $\alpha$ & $\beta$ \\
\hline $\begin{array}{c}\text { Moy } \\
\text { ET }\end{array}$ & $\begin{array}{c}0,02 \\
(0,03)\end{array}$ & $\begin{array}{c}0,10 \boldsymbol{\nabla} \\
(0,21)\end{array}$ & $\begin{array}{c}0,00 \\
(0,02)\end{array}$ & $\begin{array}{c}0,40 \boldsymbol{\nabla} \\
(0,28)\end{array}$ & $\begin{array}{c}-0,01 \\
(0,04)\end{array}$ & $\begin{array}{r}0,53 \mathbf{\Delta} \\
(0,15\end{array}$ & $\begin{array}{l}-0,04 \\
(0,04)\end{array}$ & $\begin{array}{r}0,68 \boldsymbol{\Delta} \\
(0,27)\end{array}$ & $\begin{array}{c}-0,02 \\
(0,03)\end{array}$ & $\begin{array}{c}0,23 \boldsymbol{\nabla} \\
(0,42)\end{array}$ & $\begin{array}{c}0,11 \\
(0,31)\end{array}$ & $\begin{array}{r}0,48 \boldsymbol{\nabla} \\
(0,24)\end{array}$ & $\begin{array}{c}0,07 \\
(0,04)\end{array}$ & $\begin{array}{r}0,40 \boldsymbol{\Delta} \\
(0,25)\end{array}$ \\
\hline
\end{tabular}

Source : calculs de l'auteur.

$\boldsymbol{\Delta}$ : augmentation par rapport à la période précédente (divergence).

$\boldsymbol{\nabla}$ : diminution par rapport à la période précédente (convergence).

HK : Hong Kong, Jap : Japon, Mal : Malaisie, Phil : Philippines, Thaï : Thaïlande.

Moy : moyenne ; ET : écart-type. 
Tableau 24 : convergence des chocs d'offre vers la Thaillande

\begin{tabular}{|c|c|c|c|c|c|c|c|c|c|c|c|c|c|c|}
\hline \multirow[b]{2}{*}{$94.1-2003.2$} & \multicolumn{2}{|c|}{ Chine } & \multicolumn{2}{|c|}{ Corée } & \multicolumn{2}{|c|}{ HK } & \multicolumn{2}{|c|}{ Jap } & \multicolumn{2}{|c|}{ Mal } & \multicolumn{2}{|c|}{ Phil } & \multicolumn{2}{|c|}{ Sing } \\
\hline & $\alpha$ & $\beta$ & $\alpha$ & $\beta$ & $\alpha$ & $\beta$ & $\alpha$ & $\beta$ & $\alpha$ & $\beta$ & $\alpha$ & $\beta$ & $\alpha$ & $\beta$ \\
\hline $\begin{array}{c}\text { Moy } \\
\text { ET }\end{array}$ & $\begin{array}{c}0,02 \\
(0,08)\end{array}$ & $\begin{array}{c}0,07 \\
(0,10)\end{array}$ & $\begin{array}{l}-0,22 \\
(0,26)\end{array}$ & $\begin{array}{c}0,22 \\
(0,10)\end{array}$ & $\begin{array}{l}-0,11 \\
(0,21)\end{array}$ & $\begin{array}{c}0,26 \\
(0,17)\end{array}$ & $\begin{array}{l}-0,06 \\
(0,12)\end{array}$ & $\begin{array}{c}0,57 \\
(0,41)\end{array}$ & $\begin{array}{l}-0,16 \\
(0,29)\end{array}$ & $\begin{array}{c}0,17 \\
(0,54)\end{array}$ & $\begin{array}{c}0,02 \\
(0,19)\end{array}$ & $\begin{array}{c}0,53 \\
(0,09)\end{array}$ & $\begin{array}{c}0,03 \\
(0,14)\end{array}$ & $\begin{array}{c}0,07 \\
(0,16)\end{array}$ \\
\hline $95.1-98.4$ & $\alpha$ & $\beta$ & $\alpha$ & $\beta$ & $\alpha$ & $\beta$ & $\alpha$ & $\beta$ & $\alpha$ & $\beta$ & $\alpha$ & $\beta$ & $\alpha$ & $\beta$ \\
\hline $\begin{array}{l}\text { Moy } \\
\text { ET }\end{array}$ & $\begin{array}{c}0,05 \\
(0,05)\end{array}$ & $\begin{array}{c}0,09 \\
(0,10)\end{array}$ & $\begin{array}{l}-0,28 \\
(0,17)\end{array}$ & $\begin{array}{c}0,20 \\
(0,06)\end{array}$ & $\begin{array}{l}-0,05 \\
(0,10)\end{array}$ & $\begin{array}{c}0,28 \\
(0,11)\end{array}$ & $\begin{array}{l}-0,02 \\
(0,12)\end{array}$ & $\begin{array}{c}0,37 \\
(0,50)\end{array}$ & $\begin{array}{l}-0,27 \\
(0,15)\end{array}$ & $\begin{array}{c}0,01 \\
(0,47)\end{array}$ & $\begin{array}{l}-0,04 \\
(0,15)\end{array}$ & $\begin{array}{c}0,53 \\
(0,09)\end{array}$ & $\begin{array}{c}0,09 \\
(0,11)\end{array}$ & $\begin{array}{c}0,09 \\
(0,15)\end{array}$ \\
\hline $99.1-2003.2$ & $\alpha$ & $\beta$ & $\alpha$ & $\beta$ & $\alpha$ & $\beta$ & $\alpha$ & $\beta$ & $\alpha$ & $\beta$ & $\alpha$ & $\beta$ & $\alpha$ & $\beta$ \\
\hline $\begin{array}{c}\text { Moy } \\
\text { ET }\end{array}$ & $\begin{array}{l}-0,03 \\
(0,03)\end{array}$ & $\begin{array}{c}0,03 \boldsymbol{\nabla} \\
(0,03)\end{array}$ & $\begin{array}{c}-0,03 \\
(0,03)\end{array}$ & $\begin{array}{r}0,27 \mathbf{\Delta} \\
(0,04)\end{array}$ & $\begin{array}{l}-0,07 \\
(0,05)\end{array}$ & $\begin{array}{r}0,29 \boldsymbol{\Delta} \\
(0,06)\end{array}$ & $\begin{array}{l}-0,07 \\
(0,04)\end{array}$ & $\begin{array}{r}0,67 \mathbf{\Delta} \\
(0,15)\end{array}$ & $\begin{array}{c}-0,07 \\
(0,05)\end{array}$ & $\begin{array}{r}0,33 \boldsymbol{\Delta} \\
(0,18)\end{array}$ & $\begin{array}{c}0,14 \\
(0,10)\end{array}$ & $\begin{array}{r}0,52 \boldsymbol{\nabla} \\
(0,08)\end{array}$ & $\begin{array}{c}0,05 \\
(0,03)\end{array}$ & $\begin{array}{r}0,11 \boldsymbol{\Delta} \\
(0,02)\end{array}$ \\
\hline
\end{tabular}

Source : calculs de l'auteur.

$\boldsymbol{\Delta}$ : augmentation par rapport à la période précédente (divergence).

$\boldsymbol{\nabla}$ : diminution par rapport à la période précédente (convergence).

HK : Hong Kong, Jap : Japon, Mal : Malaisie, Phil : Philippines, Sing : Singapour.

Moy : moyenne ; ET : écart-type. 